NASA TECHNICAL NOTE

o
y
0
$z$
$\vdots$
$\frac{1}{2}$

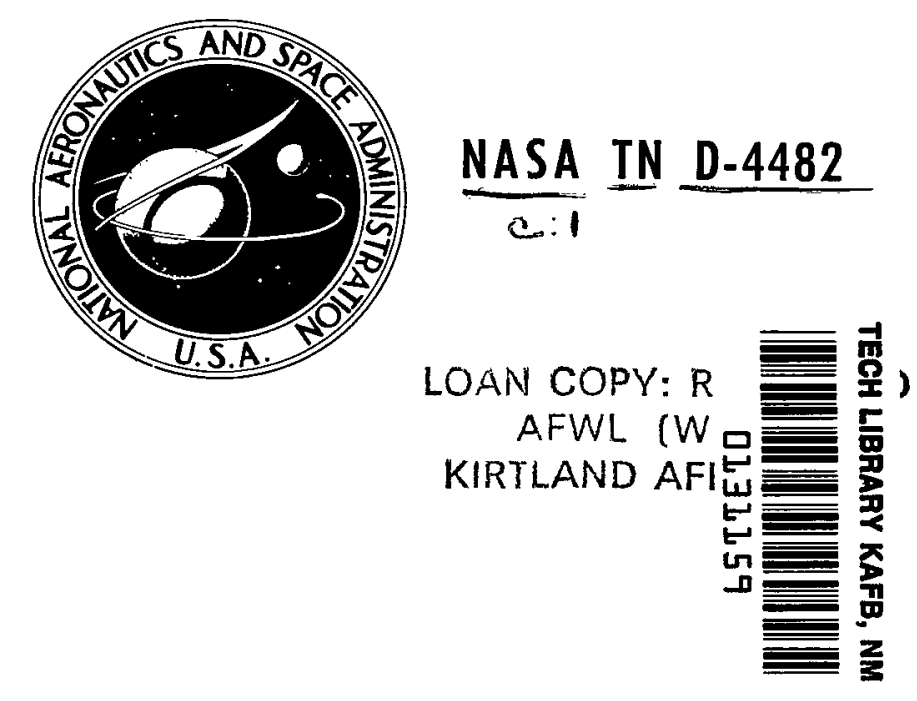

:

\title{
EXTERNAL AERODYNAMICS OF THE MAGNETOSPHERE
}

by John R. Spreiter, Alberta Y. Alksne, and Audrey L. Summers

Ames Research Center

Moffett Field, Calif.

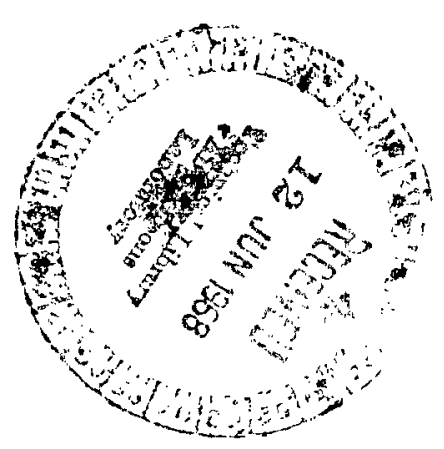

NATIONAL AERONAUTICS AND SPACE ADMINISTRATION - WASHINGTON, D. C. • JUNE 1968 
EXTERNAL AERODYNAMICS OF THE MAGNETOSPHERE

By John R. Spreiter, Alberta Y. Alksne, and Audrey L. Summers

Ames Research Center

Moffett Field, Calif. 


\title{
EXTERNAL AERODYNAMICS OF THE MAGNETOSPHERE*
}

\author{
By John R. Spreiter, Alberta Y. Alksne, \\ and Audrey L. Summers
}

Ames Research Center

SUMMARY

A comprehensive survey is given of the continuum fluid theory of the solar wind and its interaction with the Earth's magnetic field, and the relation between the calculated results and those actually measured in space. A unified basis for the entire discussion is provided by the equations of magnetohydrodynamics, augmented by relations from kinetic theory for certain small-scale details of the flow.

While the full complexity of magnetohydrodynamics is required for the formulation of the model and the establishment of the proper conditions to apply at the magnetosphere boundary, it is shown that the magnetic field actually experienced in space is usually sufficiently small that an adequate approximation to the solution can be obtained by first solving the simpler equations of gasdynamics for the flow and then using the results to calculate the deformation of the magnetic field. Numerous specific results are presented, including those for conditions in the solar wind, the shape of the magnetosphere boundary, and the flow and magnetic fields around the magnetosphere. The calculated results are compared with data obtained by numerous spacecraft, including Explorer 12, IMP-1, Pioneers VI and VII, and the Vela satellites.

\section{INTRODUCTION}

The flow of the solar wind past the Earth and its magnetosphere constitutes a problem similar in many ways to the familiar, but complex problem of the external aerodynamics of round-nosed bodies in a supersonic stream. It is the purpose of this paper to provide a résumé of some of the basic ideas, both theoretical and experimental, that support this correspondence, and to present a connected account of many of the principal quantitative results that have been obtained through application and extension of methods developed originally for the aerodynamics of reentry vehicles. In addition, the basic concepts of the theory of the solar wind itself are outlined in some detail in order to illustrate the basic unity of the entire theoretical description of the solar wind and its interaction with the geomagnetic field. Finally, extensive discussions of the internal consistency of the theory and the degree to which the results correspond to conditions observed in space are included for the purpose of providing an understanding of the overall reliability of the theory.

* Presented at the Summer Institute for Physics of the Magnetosphere, Boston College, Massachusetts, June 19-28, 1967. 
THE SOLAR WIND

Before embarking on the major topic of this paper, the interaction of the solar wind and the Earth's magnetosphere, let us review the basic properties and idealizations of the solar wind itself, many of which are summarized in figure 1. First of all, it should be recognized that it is the solar wind

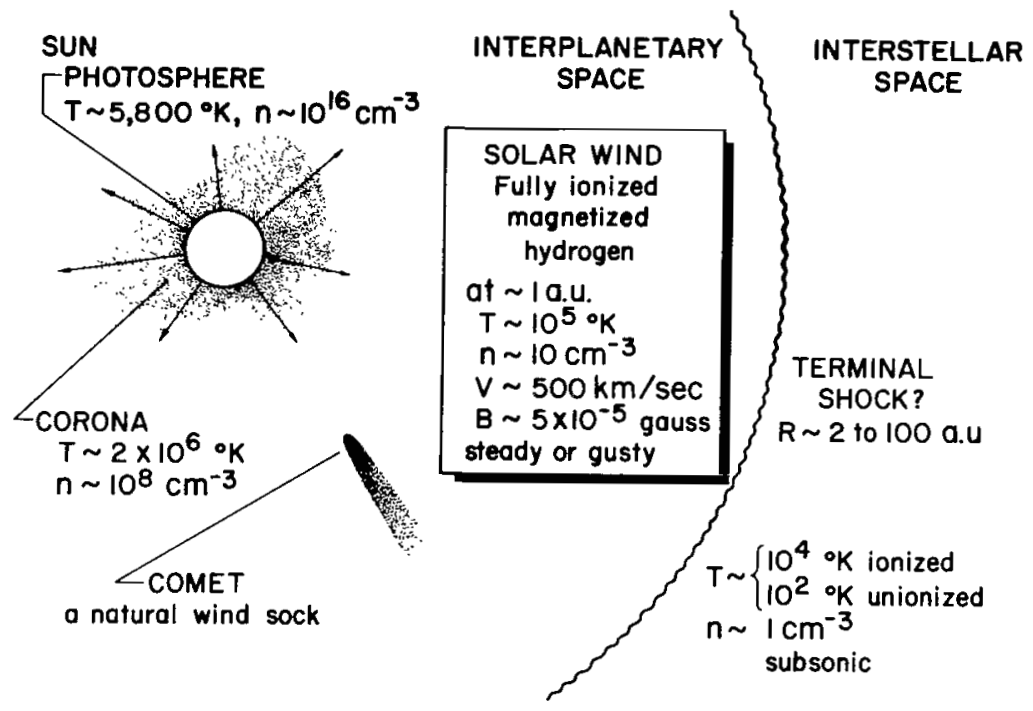

Figure 1.- The solar wind - a hypersonic gale in interplanetary space.

with its nominal velocity of 300 to $800 \mathrm{~km} / \mathrm{sec}$, and not the Earth's $30 \mathrm{~km} / \mathrm{sec}$ motion in its orbit around the Sun, that provides the dominant contribution to the relative motion of the Earth and the interplanetary plasma. In many ways, we may think of the solar wind as a hypersonic gale that blows through interplanetary space. From an overall point of view, the solar wind may be considered to result from forces in the interior of the Sun that are too large to be constrained by the feeble pressure that can be imposed by the tenuous cool gas of the interstellar medium. As the immediate source of the solar wind, we may look to the unsteady conditions in the relatively cool visible surface, or photosphere, of the Sun. Here are to be found a wide spectrum of acoustical, hydromagnetic, and internal gravity waves that grow tremendously in amplitude as they propagate upward into an atmosphere of diminishing density. The dissipation of these waves, either directly or indirectly after their culmination in shock waves, results in a substantial deposition of energy generally believed to be sufficient to account for the high temperature of the solar corona relative to the photosphere. Since the energy is being deposited in the form of heat at a greater rate than can be transported away by radiation or thermal conduction, convection in the form of the solar wind ensues. The average flow starts gradually with insignificant velocities of the order of $1 \mathrm{~km} / \mathrm{sec}$ or less in the lower corona, and increases steadily with increasing distance from the sun until its ultimate velocity is attained beyond a few tens of solar radii. The expanding coronal gas carries with it the solar magnetic field, stretching the lines of force outward through the solar system and enhancing the strength of the interplanetary field far beyond 
what it would be in a stationary atmosphere or a vacuum. Representative values for the properties of the solar wind in the vicinity of the Earth's orbit are summarized in the box in the center of figure 1 .

At great distances from the Sun, the solar wind must merge somehow into the interstellar medium. Although the distribution of interstellar gas, and its degree of ionization, is both very nonuniform and uncertainly determined, temperatures and densities of the order of those indicated on figure 1 are frequently quoted (see, for instance, Kaplan, 1966). It is generally presumed, although on the basis of scanty evidence, that a standing shock wave will develop through which the supersonic velocities of the solar wind will decelerate abruptly and become subsonic. Estimates of the location of this terminal shock wave are very uncertain at this time. It is usually placed in the range from 50 to $100 \mathrm{AU}$. A notable exception, however, is the value of $2 \mathrm{AU}$ suggested by Brandt (1962) on the basis of his analysis of the directions of comet tails (see Belton, Brandt, and Hodge (1963) and Brandt (1967) for later comments). One aspect of this terminal shock wave that does not appear to have been adequately considered in these discussions is that the temperature attained by the gas behind the terminal shock wave is far in excess of the temperatures quoted above for the interstellar medium. Whether the gas cools gradually as it continues to flow subsonically away from the sun by means of some process such as radiation not normally included in the analysis of the solar wind, remains at high temperature throughout a spherical shell terminated at still greater heliocentric distances by a second shock advancing through the interstellar medium, or behaves in some other manner altogether remains essentially unknown. It may be, on the other hand, that the supersonic solar wind terminates without the intervention of a shock wave in the manner suggested recently by Faus (1966) through the action of nonadiabatic transfer of kinetic energy to the interstellar medium in a roughly constant pressure process that takes place between about 1 to $3.5 \mathrm{AU}$.

Although geomagnetic evidence had long before led to the belief that solar plasma was projected all the way to the Earth's orbit and beyond, both occasionally from flares and more steadily in localized "M" region beams, the modern discussion of the solar wind begins with Biermann's $(1951,1957)$ analysis of the behavior of ionized comet tails. An outstanding photograph of a more recent comet, Ikeya-Seki, obtained at the Mojave Tracking Station is shown in figure 2. Clearly visible are the head of the comet near the horizon at the bottom of the photograph and the extremely elongated tail. Although it had become customary to ascribe the antisolar direction of cometary tails to radiation pressure of sunlight, Biermann showed this explanation to be inadequate in many respects, and proposed in its place the concept of an approximately radial high-speed flow of ionized gas from all parts of the Sun at all times. It is not difficult to accept this explanation of a photograph like that of figure 2, since the general effect is very remindful of smoke from a smudge pot or of a wind sock in a strong and steady wind.

Although the solar corona is not normally visible because of the overwhelming brilliance of the light from the photosphere, it is clearly visible, even to the unaided eye, at the time of a total eclipse of the Sun by the Moon. As a result, much of what is known about the solar corona has been 


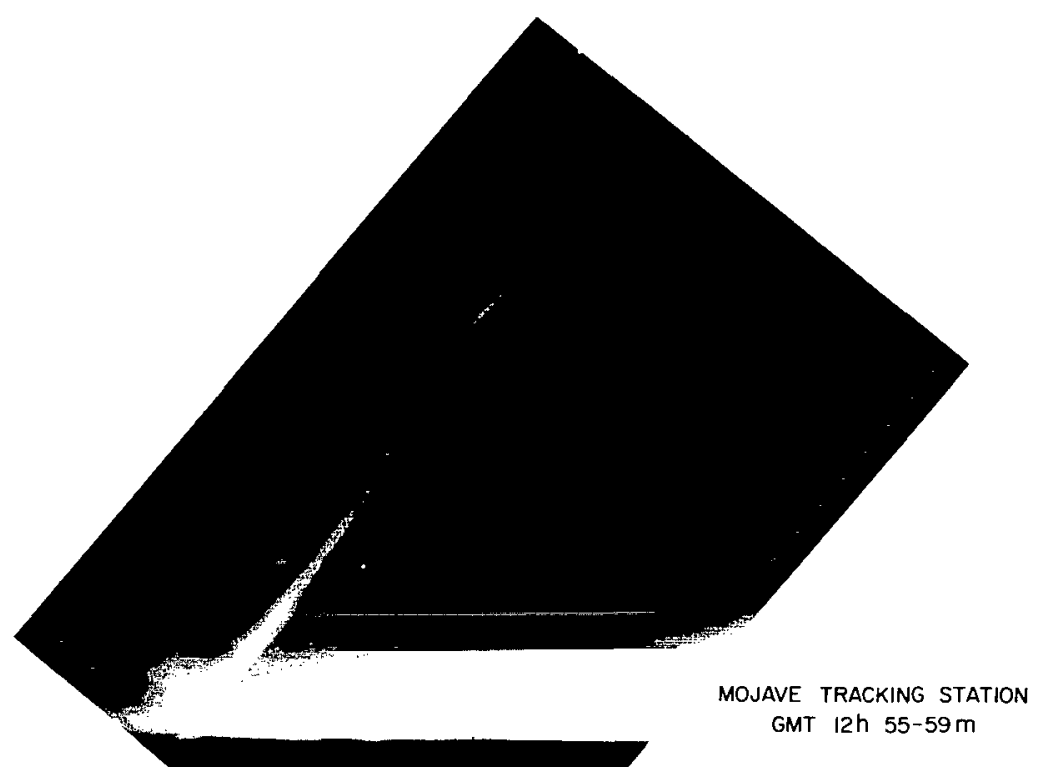

Figure 2.- Comet Ikeya-Seki - October 29, 1965.

learned from observations made during the few minutes duration of such events. In more recent years, such observations, particularly of the parts closer to the Sun, have been augmented by those taken at other times with the aid of a coronagraph, a telescope system fitted with special obscuring equipment invented by Lyot in 1937. Figure 3 shows a photograph of the corona taken during the eclipse of May 30,1965, from a NASA Convair 990 jet airliner at 38,000 feet altitude (Smith, Henderson, and Torrey, 1967). Clearly visible

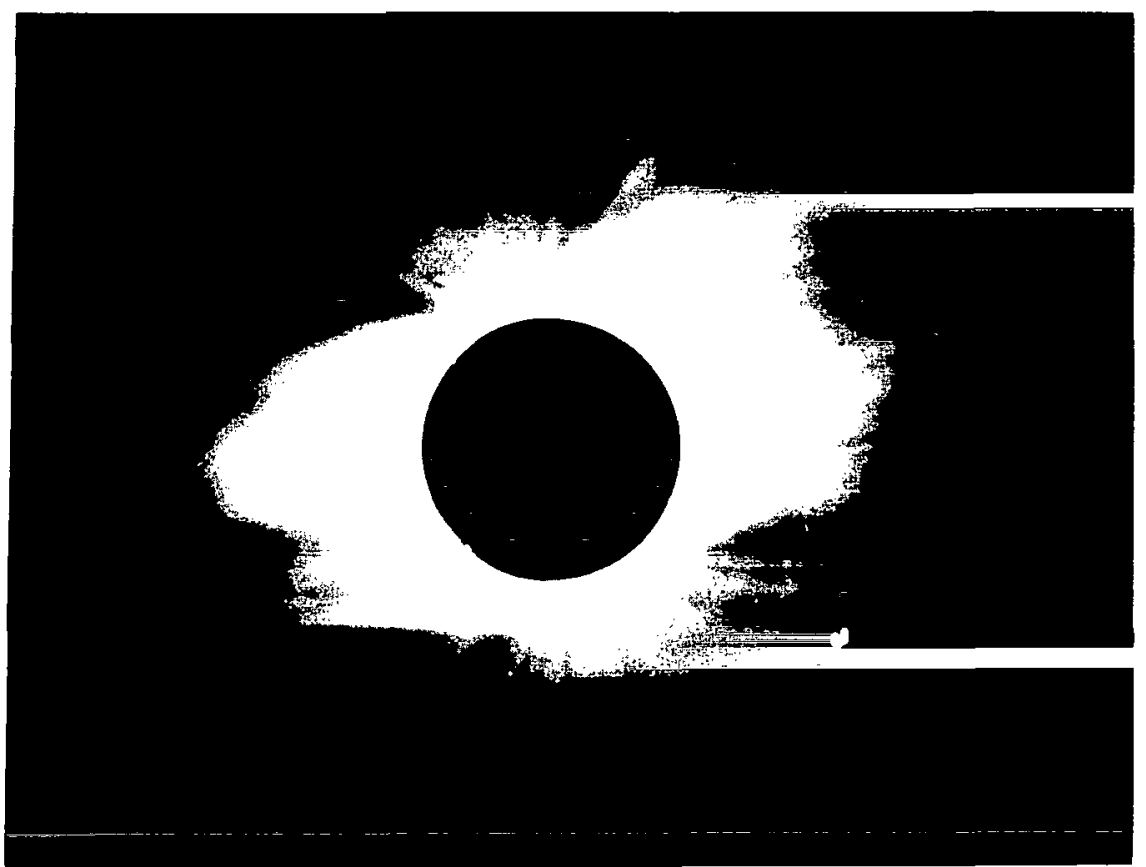

Figure 3.- Solar corona during eclipse of May 30, 1965, from NASA CV-990 aircraft at 38,000 feet. 
beyond the disk of the Moon are the polar rays, similar in shape to the magnetic lines of force at the poles of a magnetized sphere. More interesting from the present point of view is the large outward extension of the corona from the equatorial and middle latitudes. Although these plumes appear to extend only a few solar radii in this photograph, other photographs taken during other eclipses have shown rather similar features visible to distances as much as five or ten times farther from the Sun. Observations indicate that the coronal gas in these plumes is both somewhat hotter and many times denser than over the poles.

In spite of the obvious deviations from spherical symmetry in the solar corona, and presumably in the solar wind as well, the theory of the solar wind is generally based for reasons of mathematical tractability on the assumption of radially symmetric flow. The elements of this theory, which was initially presented by Parker (1958) and summarized in more developed form by Parker (1963, 1965), Lüst (1963, 1965), Scarf (1964), Dessler (1967), and others, is outlined in figure 4. Perhaps the most fundamental concept is that of treating the solar wind, even as it becomes extremely rarefied far beyond the orbit

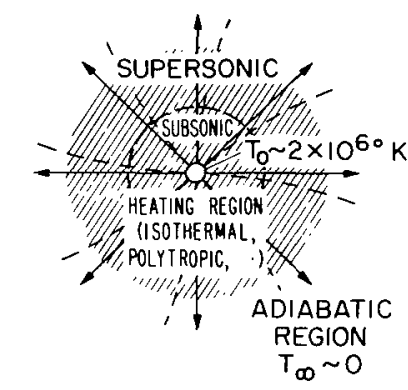

- STREAMLINES

- MAGNETIC FIELD LINES MHD $\frac{\rho v^{2}}{2}>\frac{H^{2}}{8 \pi}=\left\{\begin{array}{l}\text { GASDYNAMIC } \\ \text { FLOW } \\ \text { CONVECTED } \\ \text { MAGNETIC } \\ \text { FIELD }\end{array}\right.$
FLOW EQUATIONS

(STEADY RADIALLY SYMMETRIC FLOW)

$$
\begin{aligned}
& \text { CONTINUITY } \\
& \text { pur } 2 \text { a CONST } \\
& \rho v \frac{d v}{d r}+\frac{d p}{d r}+\frac{G M_{\odot} \rho}{r^{2}}=0 \\
& \frac{p / p_{o}}{\left(\rho / \rho_{o}\right)^{\alpha}}=\text { CONST } \\
& \alpha=1 \text { ISOTHERMAL } \\
& \alpha=\gamma=5 / 3 \text { ADIABATIC } \\
& 1<\alpha<5 / 3 \text { INTERMEDIATE }
\end{aligned}
$$

DIRECTION OF MAGNETIC FIELD

$$
\frac{H_{\theta}}{H_{r}}=\frac{\omega_{\odot} r}{V}
$$

Figure 4.- Elements of solar wind theory (Parker, 1958).

of the Earth, as an ordinary continuum gas. The basic equations are thus the continuity, momentum, and energy equations of gasdynamics for a radially symmetric outflow of a perfect gas from a centered gravitational field. The first two of these are indicated on figure 4, in which $\rho$ is the density, $v$ the velocity, and $p$ the pressure of the solar wind, $r$ is the heliocentric distance, $M_{\odot}=2 \times 10^{33} \mathrm{gm}$ is the mass of the Sun, and $G=6.67 \times 10^{-8} \mathrm{~cm}^{3} / \mathrm{gm} \mathrm{sec}^{2}$ is the gravitational constant. Lack of understanding of all contributions, such as that of propagating waves, to the energy flux precludes writing a reliable energy equation to complete the system. In its place, a variety of substitutes have been employed. Among the simplest are the extremes of isothermal flow within the first few solar radii from the Sun, and adiabatic flow beyond. Next in simplicity is the generalization to a polytropic relation $\left(\mathrm{p} / \mathrm{p}_{\mathrm{o}}\right) /\left(\rho / \rho_{\mathrm{o}}\right)^{\alpha}$, in which $\alpha$ is an arbitrary constant. The boundary 
conditions usually employed with these equations are supplied by selecting representative values, such as those indicated on figure 1 , for the temperature and density at the base of the corona, and by requiring that the velocity be supersonic, or the temperature or pressure approach zero, at very great distances from the Sun. To these must be added the requirement that the transition from subsonic to supersonic flow must be achieved with a continuous acceleration. If this condition is not observed, a forbidden expansion shock wave through which the entropy would decrease would be permitted by the governing equations.

Although the equations for the flow of the solar wind are those of gasdynamics, it has been recognized from the outset that the gas is fully ionized, and that the relatively weak interplanetary magnetic field would be convected about by the solar wind. Combination of the radially symmetric outflow of the gas with the approximately 27-day rotation period of the feet of the field lines anchored in the solar surface leads through this concept, formalized by the relation on the bottom of figure 4 for the direction of the magnetic field $\underset{\sim}{\mathrm{H}}$ in terms of the velocity $\underset{\sim}{\mathrm{v}}$ of the solar wind and the angular velocity ${ }^{\sim} \odot$ of the Sun, to an understanding of the normal spiral "hose-angle" configuration of the interplanetary magnetic field. Although the configuration of the interplanetary magnetic field is thus primarily determined by and secondary to the gas flow, it is of vital importance in guiding and deflecting the motion of the relatively rare, but very energetic, particles that constitute the galactic and solar cosmic rays.

Introduction of the polytropic pressure-density law permits integration in analytic form of the equations for the solar wind shown on figure 4 . Parker (1963) has shown that the solution indicates the velocity will go to zero near the Sun only if the polytropic index $\alpha$ is less than 3/2. Furthermore, the solution can only be extended to infinity if the ratio $\mathrm{R}_{\mathrm{O}} \mathrm{p}_{\mathrm{O}} / \mathrm{GM}_{\odot \mathrm{O}_{\mathrm{O}}}=2 \mathrm{R}_{\mathrm{O}} \mathrm{n}_{\mathrm{O}} \mathrm{kT} \mathrm{T}_{\mathrm{O}} / \mathrm{GM}_{\odot} \mathrm{O}_{\mathrm{O}}$ of the thermal energy to the gravitational potential energy at the base of the corona is greater than $(\alpha-1) / \alpha$. The quantity $R_{0}$ represents the heliocentric distance to the assumed base of the corona, and $n$ represents the number density of the ions. For fully ionized hydrogen, $\mathrm{n}=\rho / \mathrm{m}_{\mathrm{p}}$ in which $\mathrm{mp}_{\mathrm{p}}=1.67 \times 10^{-24} \mathrm{gm}$ is the mass of a proton. Parker also showed that $\mathrm{R}_{\mathrm{O}} \mathrm{P}_{\mathrm{O}} / \mathrm{GM}_{\odot} \rho_{\mathrm{O}}$ must not exceed $1 / 2 \alpha$ since no stationary solution exists beyond this limit. If the thermal energy should exceed $1 / 2 \alpha$ times the gravitational potential energy at the base of the corona, expansion of a more explosive character would presumably occur. Within these limits, there still remains a substantial family of solutions, each of which involves extended heating since $\alpha<5 / 3$.

Integral curves representing the solution, for a polytropic index $\alpha$ within the above bounds, for the variation of the absolute value of the velocity $v$ of the solar wind with distance $r$ from the sun are illustrated in figure 5. The singularity in the solution at the point where the two heavy integral curves cross occurs where the velocity is equal to the speed of sound. It is usually located within a few solar radii of the sun when representative values are used for the boundary conditions. Various of these curves, with either plus or minus sign on $v$ to represent outflow or inflow, have been used at one time or another to explain or propose significant 


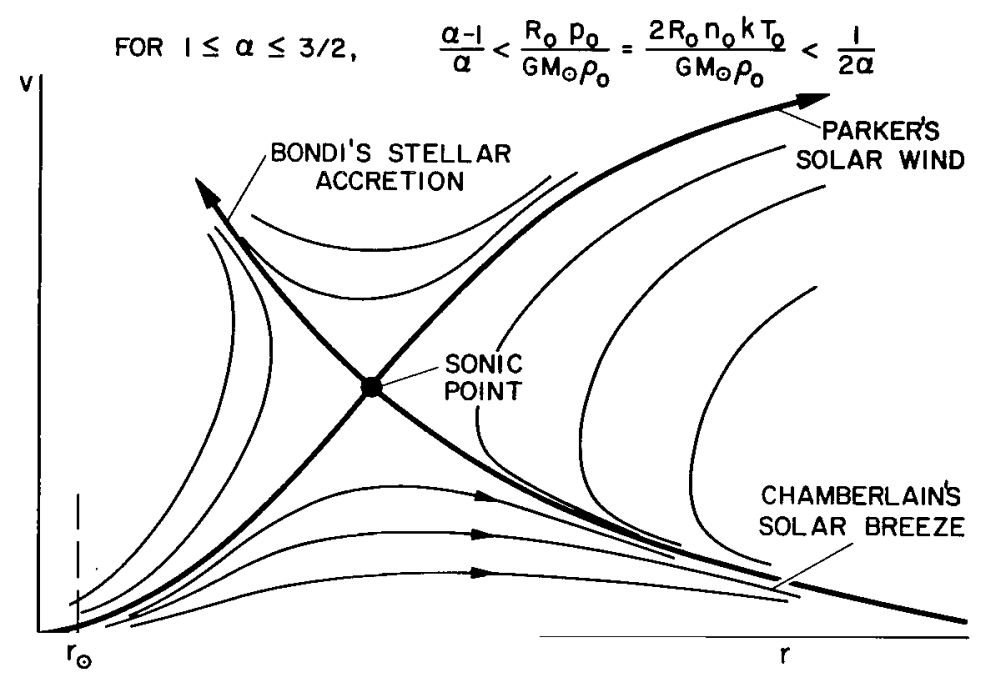

Figure 5.- Sketch of solutions for variation of solar wind velocity with distance from Sun. interplanetary phenomena.

Earliest is the investigation of Bondi (1952) of steady spherically symmetric accretion by a star of a perfect interstellar gas considered to be at rest at infinity. He selected, therefore, the indicated heavy curve to represent an accretion flow that smoothly accelerates from subsonic speeds at infinity to supersonic speeds nearer the Sun. McCrea (1956) later added the feature of a standing shock wave to terminate the supersonic flow before it reached the sensible surface of the star.

In the solar system, however, Biermann's analysis of

the behavior of comet tails was meanwhile strongly suggesting that the flow was not only outward from the Sun, but at high speed. In view of this, and also the smallness of the confining pressure that could be maintained by the very tenuous interstellar gas, and the even more tenuous, although energetic, cosmic-ray gas, Parker considered outflow and selected the indicated heavy integral curve to represent a continuous acceleration of the solar gas from subsonic speeds near the Sun to supersonic speeds farther away. Although precedents were numerous in gasdynamics as well as other fields, theoretical objections were raised by Chamberlain (1961) to the selection of an integral curve that passes through the singular point. He advocated instead selection of one of the lower set of curves that go to zero both at the origin and at infinity, and never attain sonic velocity at any point. Because the resulting flow is about 10 times slower at the Earth's orbit than that indicated by Parker's solar-wind theory, the results were referred to as Chamberlain's solar-breeze theory. Although it was difficult to determine definitively the relative merits of the two proposals at the time, data obtained with many spacecraft subsequently have confirmed decisively that the supersonic solution chosen by Parker is the appropriate one for at least the portion of the solar system within the orbit of Mars, at approximately $1.5 \mathrm{AU}$.

Figure 6 shows one of the first results to emerge from Parker's study of the solar wind, the run of velocity with distance from the sun for a variety of assigned coronal temperatures. Although the calculations were made with the oversimplified assumption of isothermal flow, the results show two characteristics that have remained essentially unchanged in all subsequent refinements of solar-wind theory. They are that the solar wind accomplishes most of its acceleration within the first 10 or 20 solar radii, and that velocities of the order of several hundred kilometers per second at the orbit of Earth are compatible with coronal temperatures of the order of a few million degrees Kelvin. 


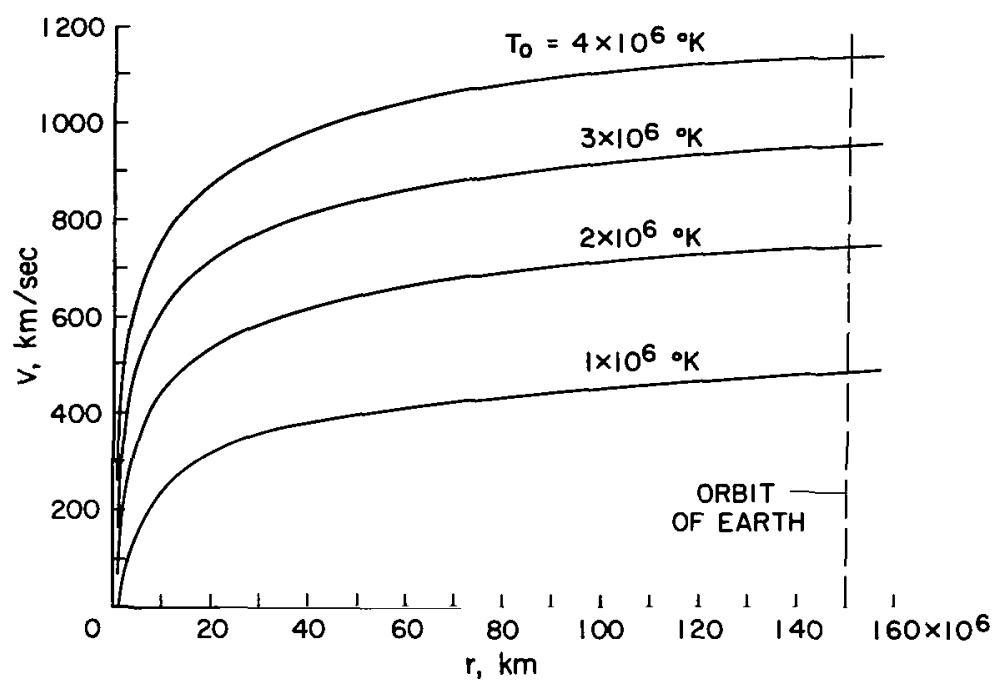
Figure 6. - Effect of coronal temperature on solar wind velocity -
isothermal mode1 (Parker, 1958, 1963).

Many refinements and elaborations of the theory of the solar wind have been made during the years since its first announcement. Although it is not the purpose of this paper to present a comprehensive review of the theory of the solar wind, a brief outline of some of the salient features of successive developments of the theory is presented in figure 7 . First of al1, we may note that nearly all theories of the solar wind are based on the equations of a one-component perfect gas. The earliest and simplest version of the theory was based on the assumptions of isothermal flow for the first several solar radii, and adiabatic flow thereafter to infinity. Since the maintenance of a constant temperature in an expanding gas requires a substantial supply of heat throughout the isothermal region, this version of the theory may be characterized as having an extended heating region. The region in which heat must be supplied to maintain the flow is even more extensive in the versions of the theory that employ the polytropic pressure-density relationship because the divergence of the energy flux is proportional to $5-3 \alpha$, and is hence positive for all $\alpha<5 / 3$. Since the polytropic theory is often employed all the way from the Sun to infinity, with either constant or variable $\alpha<5 / 3$, the extent of the implied heating region may be very large indeed.

ONE FLUID THEORIES

$$
\begin{aligned}
& \text { EXTENDED HEATING REGION } \\
& \text { ISOTHERMAL + ADIABATIC } \\
& \text { POLYTROPIC PRESSURE-DENSITY RELATIONSHIP } \frac{p / p_{0}}{\left(p / p_{0}\right)^{\alpha}}=\text { CONST } \\
& \alpha=\text { CONST } \\
& \alpha=f(r)
\end{aligned}
$$

TWO FLUID THEORY HEAT CONDUCTION

$$
\text { - . • . }
$$

Figure 7.- Successive developments in solar wind theory. 
Although the damping of waves that are simultaneously tending to grow in amplitude as they propagate away from the Sun into a region of diminishing density has always remained a possible mechanism for the production of the required heat, early estimates suggested the possibility that thermal conduction may be the principal process, in addition to convection by the solar wind, by which energy is conveyed from the wave-damping region to higher portions of the corona. According to this view, it is considered that the shock waves that provide the principal heating of the corona are, in effect, absorbed completely in a thin heating region in the lower part of the corona, and that the thermal conductivity of the hot coronal gases is sufficiently great that the heat required at greater distances to drive the solar wind can be supplied by heat conduction. Later refinements of this approach have been to add effects of viscosity, rotation, and magnetic fields, but these do not usually alter the principal features of the flow to any substantial degree.

It has been customary in the theories of the solar wind that include effects of thermal conduction and/or viscosity to use the expressions given by Spitzer (1956, 1962) to calculate the transport coefficients. The analysis leading to these expressions also indicates that the equipartition time required for protons and electrons to reach the same temperature tends to be of the order of a year when conditions representative of those at the Earth's orbit are used. Using this result, Sturrock and Hartle (1966) have observed that one should expect the proton and electron temperatures to be quite different in the solar wind, and proceeded to develop a two-fluid model to allow for this effect. Although data from Vela II satellite (Strong, Asbridge, Bame, Heckman, and Hundhausen, 1966) show that large differences between the temperatures of the protons and electrons may occasionally occur in the solar wind, the temperatures measured most of the time tend to be in better agreement with the predictions of the previous single-fluid theories than with those of the two-fluid theory. Sturrock and Hartle offer several suggestions to account for the apparent heating of the solar-wind protons by a nonthermal energy source operative in interplanetary space as far out from the Sun as the Earth's orbit. The possibility they prefer is that protons are heated by stochastic acceleration following the development of turbulence in the solar wind by an unspecified instability mechanism.

\section{MATHEMATICAL REPRESENTATION OF THE INTERACTION OF THE SOLAR WIND AND THE GEOMAGNETIC FIELD}

Just as in the case of the solar wind itself, the fundamental assumption upon which this discussion is based is that the average bulk properties of the flow can be described adequately by the continuum equations for a perfect gas. No attempt will be made here to justify this assumption in detail, except to remark that the presence of a weak and irregular magnetic field in the incident solar-wind plasma appears to be sufficient to couple the motions of the particles even in the absence of collisions, and that numerous data obtained in space display outstanding agreement with results calculated on this basis. Because of the important role of the magnetic field of the Earth in the interaction with the solar wind, the simplest mathematical model capable of 
adequate description of many of the dominant features is that provided by the standard magnetohydrodynamic equations for steady flow of a dissipationless perfect gas (Spreiter, Summers, and Alksne, 1966).

The governing differential equations are thus as illustrated in figure 8 . The symbols $\rho, p, S$, and $v$ refer to the density, pressure, entropy, and velocity of the gas, $\underset{\sim}{H}$ refers to the magnetic field, and $\gamma=c_{p} / c_{v}=(N+2) / N$ in which $c_{p}$ and $c_{v}$ are constants representing the specific heats at constant
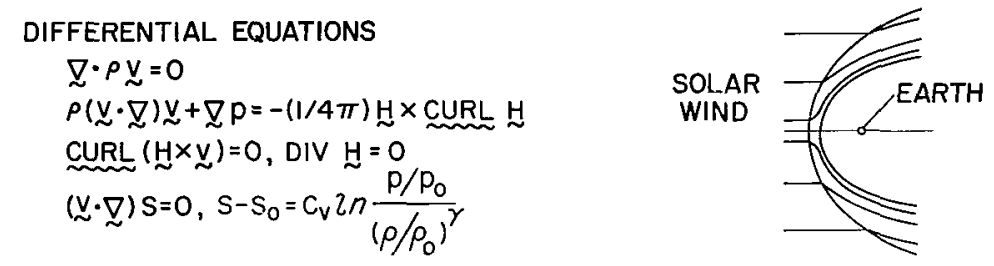

$$
\begin{aligned}
& \text { CONSERVATION EQUATIONS FOR DISCONTINUITIES } \\
& {\left[\rho V_{n}\right]=0} \\
& {\left[\rho V_{n} \underset{\sim}{\vee}+\left(p+H^{2} / 8 \pi\right) \hat{n}-H_{n} \underset{\sim}{H} / 4 \pi\right]=0} \\
& {\left[H_{n} V_{\dagger}-H_{\dagger} V_{n}\right]=0,\left[H_{n}\right]=0} \\
& {\left[\rho V_{n}\left(h+v^{2} / 2\right)+V_{n} H^{2} / 4 \pi-H_{n} \underset{\sim}{\vee} \cdot \underset{\sim}{H} / 4 \pi\right]=0} \\
& {[Q]=Q_{1}-Q_{0}}
\end{aligned}
$$

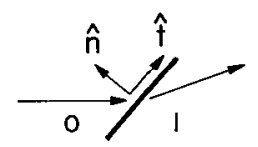

Figure 8.- Hydromagnetic equations for steady flow of a dissipationless perfect gas.

pressure and constant volume, and $N$ represents the number of degrees of freedom of the gas particles. For a monatomic gas, $N=3$, and $\gamma=5 / 3$. The temperature $T$ is related to the pressure and density by the equation of state, $\mathrm{p}=\rho \mathrm{RT} / \mu$, in which $\mathrm{R}=\left(\mathrm{c}_{\mathrm{p}}-\mathrm{c}_{\mathrm{v}}\right) \mu=8.315 \times 10^{7} \mathrm{ergs} / \mathrm{gm}{ }^{\circ} \mathrm{K}$ is the universal gas constant, and $\mu$ is the mean molecular weight nondimensionalized so that $\mu=16$ for atomic oxygen. Thus $\mu=1 / 2$ for fully ionized hydrogen plasma. The electromagnetic parts of these equations are expressed in terms of gaussian units. Since $\underset{\sim}{H}=\underset{\sim}{B}$ in such units, these two symbols will be used interchangeably in this paper in order to facilitate further comparisons with the original sources.

Although only first derivatives appear in the system of differential equations shown in figure 8 , the neglected dissipative terms are described by second derivatives. Their neglect requires that the gradients be small. In magnetohydrodynamics, as in gasdynamics, however, compressions tend to coalesce and steepen into shock waves of such small thickness that they can be considered as virtual discontinuities. In addition, attraction between like currents tends to cause current distributions to collapse into thin sheaths, across which the magnetic field can be considered in the same sense to be nearly discontinuous. Mathematically, continuous solutions of the dissipationless differential equations cease to exist, and the flow is no longer governed solely by the differential equations shown on figure 8 . Mass, momentum, magnetic flux, and energy must still be conserved, however, and the conservation equations provided in figure 8 must hold between quantities on the two sides of any such discontinuity. The symbol $h$ in the energy equation represents the enthalpy, which is related to the internal energy $\mathrm{e}=\mathrm{c}_{\mathrm{V}}^{\mathrm{T}}$ according to 
$h=c_{p} T=c_{v} T+p / \rho$. Unit vectors normal and tangential to the discontinuity surface are designated by $\hat{n}$ and $\hat{t}$, and components of $\mathcal{V}$ and $\underset{\sim}{H}$ in these directions are indicated by subscripts $n$ and $t$. The square brackets are used to indicate the difference between the enclosed quantities on the two sides of the discontinuity, as in $[Q]=Q_{1}-Q_{0}$ where subscripts 0 and 1 refer to conditions on the upstream and downstream sides of the discontinuity.

Five classes of discontinuities are described by the solutions of the conservation equations. As indicated in figure 9, those that lie along streamlines $\left(v_{n}=0\right)$ are called tangential discontinuities or contact discontinuities according to whether or not the normal component $\mathrm{H}_{\mathrm{n}}$ of the magnetic field

TANGENTIAL

$$
\begin{gathered}
v_{n}=H_{n}=0,\left[\underline{y}_{t}\right] \neq 0,\left[{\underset{H}{t}}_{t}\right] \neq 0,[\rho] \neq 0 \\
{\left[p+H^{2} / 8 \pi\right]=0}
\end{gathered}
$$

CONTACT

$$
v_{n}=0, H_{n} \neq 0,[\underline{v}]=[\underset{\sim}{H}]=[p]=0,[\rho] \neq 0
$$

ROTATIONAL

$$
\begin{aligned}
& v_{n}= \pm H_{n} /(4 \pi \rho)^{1 / 2},\left[{\underset{\sim}{f}}_{f}\right]=\left[\underset{H_{f}}{H_{f}}\right] /(4 \pi \rho)^{1 / 2} \\
& {[\rho]=[p]=\left[v_{n}\right]=\left[v^{2}\right]=\left[H^{2}\right]=\left[H_{n}\right]=0}
\end{aligned}
$$

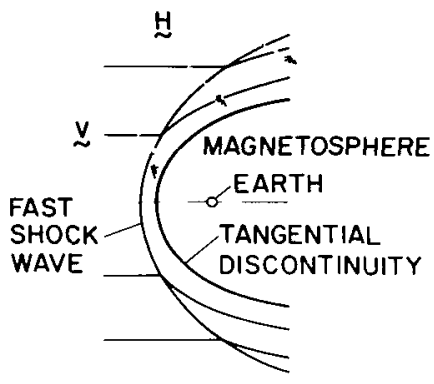

SHOCK WAVES, FAST AND SLOW

$$
\begin{aligned}
& v_{n} \neq 0,[\rho]>0,[p]>0,\left[H_{n}\right]=0 \\
& \left(\rho v_{n}\right)_{\text {FAST }} \geq\left(\rho v_{n}\right)_{\text {ROT. }} \geq\left(\rho v_{n}\right)_{\text {SLOW }} \\
& H_{\sim} \text { and } H^{2}\left(\begin{array}{l}
\text { INCREASE } \\
\text { DECREASE }
\end{array}\right) \text { THROUGH }\left(\begin{array}{c}
\text { FAST } \\
\text { SLOW }
\end{array}\right) \text { SHOCK WAVES }
\end{aligned}
$$

Figure 9.- Hydromagnetic discontinuities.

vanishes. Discontinuities across which there is flow $\left(v_{n} \neq 0\right)$ are divided into three categories called rotational discontinuities, and fast and slow shock waves. Some properties that distinguish the various discontinuities are summarized on figure 9 .

Of the various possibilities, only the tangential discontinuity has properties compatible with those required to describe a boundary surface that separates the geomagnetic field and the external flow. As in the classical Chapman-Ferraro theory of the magnetosphere boundary based on particle concepts, the condition $\mathrm{H}_{\mathrm{n}}=0$ holds and requires that there is no connectivity between the geomagnetic and interplanetary fields. Although the condition $v_{n}=0$ prohibits flow across the boundary, arbitrary differences in the density and the velocity and magnetic field components paralle1 to the boundary are permitted. It is only required that the sum of the gas pressure $p$ and the magnetic pressure $H^{2} / 8 \pi$ be the same on the two sides of the discontinuity.

Two important parameters that characterize the flow at any point are the Mach number $M=v / a$ and the AIfvén Mach number $M_{A}=v / A$. The former is the ratio of the flow velocity to the speed of sound

$$
a=(\partial p / \partial \rho)^{1 / 2}=(\gamma p / \rho)^{1 / 2}=(\gamma R T / \mu)^{1 / 2}
$$


The latter is the corresponding ratio to the speed $A=\left(H^{2} / 4 \pi \rho\right)^{1 / 2}$ of a rotational or Alfvén wave propagating along the direction of the magnetic field. Values for $a$ and A for conditions typical of those in the solar wind in the vicinity of the Earth's orbit are illustrated in figure 10. Although it is possible for weak disturbances to travel as fast as $\left(\mathrm{a}^{2}+\mathrm{A}^{2}\right)^{1 / 2}$, which they do in a direction normal to the magnetic field, the

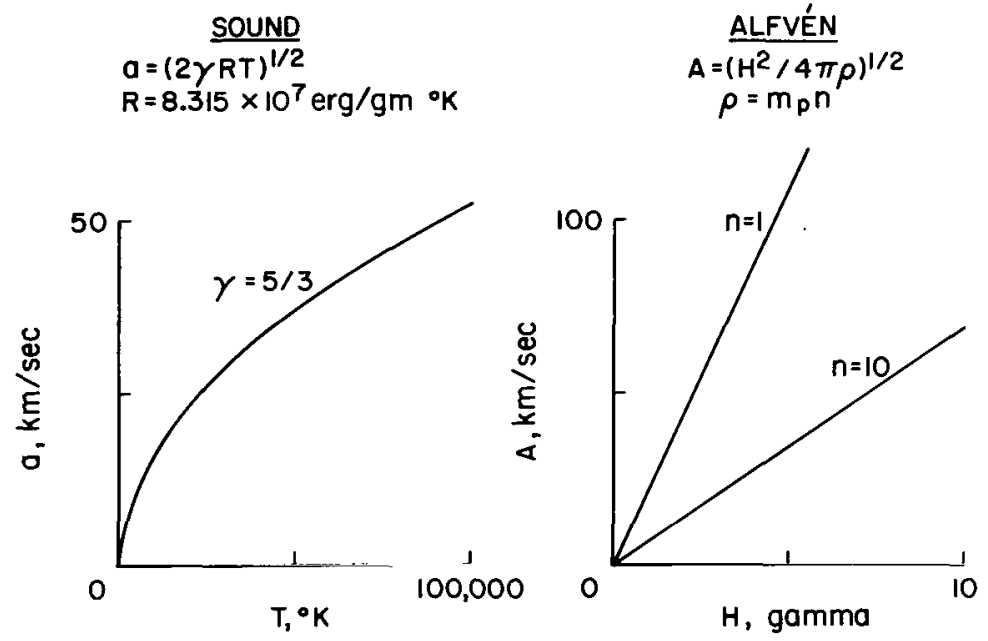

MAXIMUM PROPAGATION VELOCITY OF A WEAK HYDROMAGNETIC WAVE $=\left(a^{2}+A^{2}\right)^{1 / 2}$

Figure 10.- Significant hydromagnetic speeds for fully ionized hydrogen.

maximum propagation speed is much less than the 250 to $800 \mathrm{~km} / \mathrm{sec}$ speed of the solar wind relative to the Earth. It may be inferred immediately that a standing shock wave must exist in the flow around the Earth and its magnetosphere. Of the five classes of discontinuities outlined on figure 9, only the fast shock wave can be used to represent the Earth's bow wave, since the mass flow through each of the others is too small.

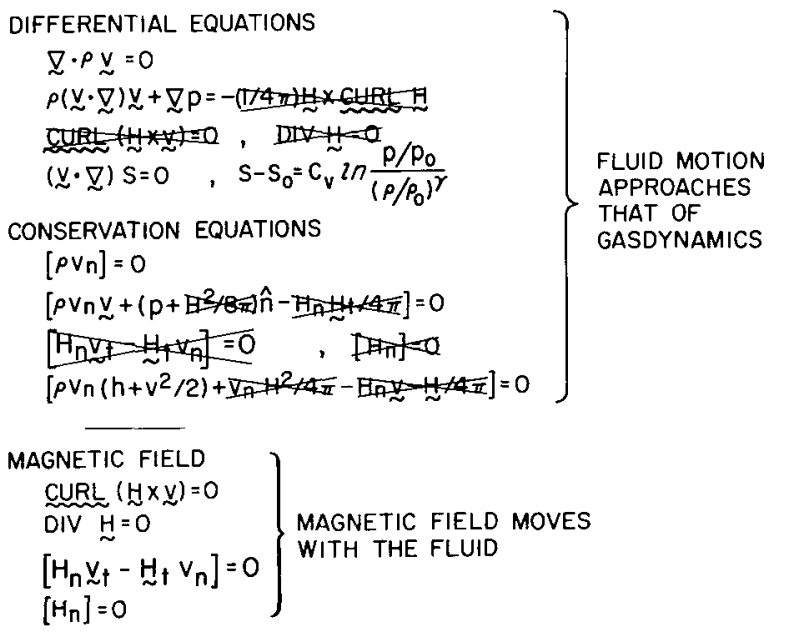

Figure 11.- Decoupling of equations for large Alfvén Mach number $M_{A}=\left(4 \pi p v^{2} / H^{2}\right)^{1 / 2}$.
An important simplification of the magnetohydrodynamic equations shown on figure 8 can be made on the basis of the large magnitude of the Alfvén Mach number. This is because the order of magnitude of the inertia term $\rho(y \cdot \underset{\sim}{\nabla}) y$ in the differential equation for the momentum is related to that of the magnetic term $(1 / 4 \pi) \underset{\sim}{H} \times \underset{\sim}{\operatorname{cur}} \underset{\sim}{1} \underset{\sim}{H}$ by the square of the Alfvén Mach number. When the latter is large, therefore, the magnetic term can usually be safely dropped from the equation, as indicated in figure 11, with little loss of accuracy. Although factors of 2 appear when similar comparisons are made of the magnetic and inertia 
terms in the conservation equations for the momentum and energy, the genera1 conclusion that the magnetic terms may be dropped from these equations when the Alfvén Mach number is large still holds. An immediate consequence is that both the differential equations and the conservation equations defining the flow are decoupled from those involving the magnetic field. Thus, a11 the properties of the flow except the magnetic field $\underset{\sim}{\mathrm{H}}$ can be determined by solving the equations of gasdynamics, and the magnetic field can be determined subsequently by solving the remaining equations using the values for $\underset{\sim}{V}$ already determined. Although certain ambiguities reside in the statement, the latter equations may be interpreted as indicating that the magnetic field lines move with the fluid, the general effect being essentially similar to that of a line of smoke or dye released at some instant into a medium flowing at speeds high relative to the diffusion rate of the contaminant. In this way, one achieves the same decoupling of the calculation of the flow from that of the magnetic field as was indicated previously in figure 1 for the solar wind itself. The main difference is that the equations for the latter are greatly simplified by restriction to radially symmetric flow, although complicated somewhat by the need to consider the gravitational attraction of the Sun and nonadiabatic flow.

At the magnetosphere boundary, a simplification of great practical utility can be made by introducing certain approximations to the tangential discontinuity conditions. The basis for these is the fact indicated on figure 12 that estimates of the magnitudes of the gas pressure $p$ and the magnetic pressure $H^{2} / 8 \pi$ show that $p$ is much less than $H^{2} / 8 \pi$ in the outer magnetosphere, and that $p$ tends to be much larger than $\mathrm{H}^{2} / 8 \pi$ in the flow around the forward part of the magnetosphere. As far as the flow exterior to the magnetosphere is concerned, the discontinuities at the magnetosphere boundary may thus be approximated satisfactorily by those of the limiting case of a tangential discontinuity in which there is a vacuum on one side, and no magnetic field on the other side.

It has also been shown (Spreiter, Summers, and Alksne, 1966) that the gas pressure of the flow on the magnetosphere boundary can be approximated

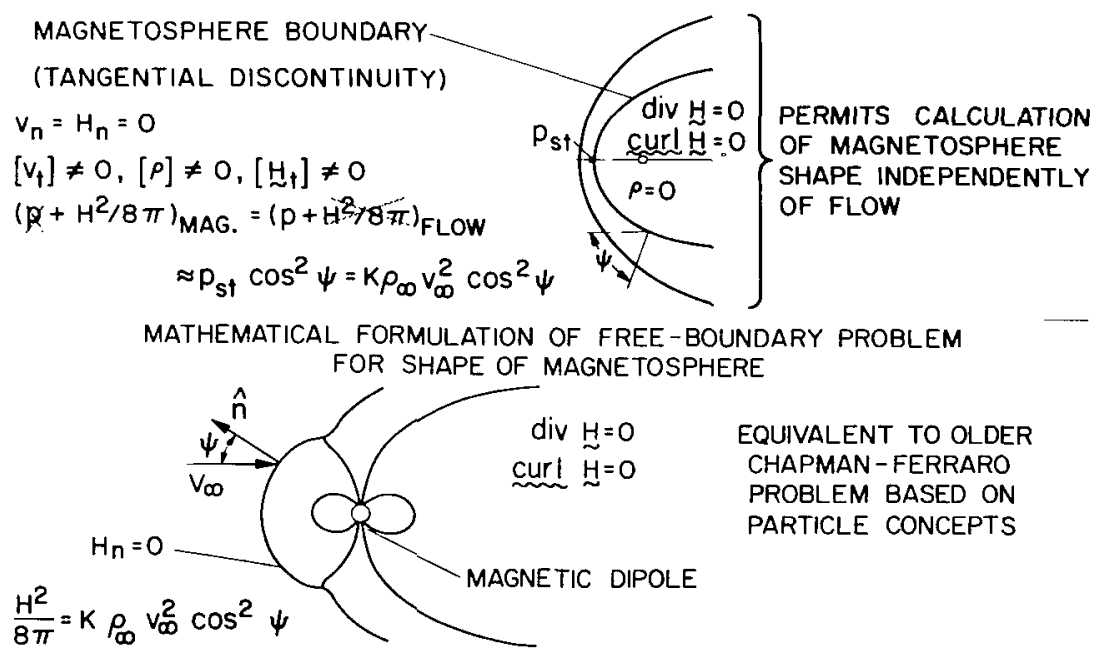

Figure 12.- Decoupling of calculations of magnetosphere shape and surrounding flow. 
adequately by the simple Newtonian formula $p=p_{s t} \cos ^{2} \psi$ where $\psi$ is the angle illustrated on figure 12 between the outward normal to the boundary and the flow direction of the undisturbed incident solar wind, and $\mathrm{p}_{\mathrm{st}}=\mathrm{K}_{\infty} \mathrm{v}_{\infty}{ }^{2}$ is the stagnation pressure exerted on the nose of the magnetosphere. The symbols $\rho_{\infty}$ and $v_{\infty}$ in the latter expression refer to the density and velocity of the solar wind as it approaches the bow wave. The constant $K$ is usually taken to be unity, but yields better accuracy if equated to 0.88 for $\gamma=5 / 3$. It is implicit in the use of this expression that $\cos \psi \geq 0$, since otherwise the surface would be shielded from the flow. The net result of the introduction of the Newtonian approximation is that the calculation of the shape of the magnetosphere boundary is decoupled from the detailed analysis of the surrounding flow. Of further significance is the fact that the resulting mathematical problem defined here on figure 12 is identical to that of the classical Chapman-Ferraro theory based on quite different concepts of the operative physical processes.

In this way, the theoretical study of the external aerodynamics of the magnetosphere may be separated into three simpler parts without incurring undue error. First of all, the shape of the magnetosphere boundary may be considered to be the same as indicated by the Chapman-Ferraro theory. Second, the flow around the magnetosphere may be determined by solving the equations of gasdynamics for flow around the shape given by the solution of the ChapmanFerraro problem. Finally, the magnetic field in the region exterior to the magnetosphere may be determined by use of the concept that the field moves with the fluid.

THE SHAPE OF THE MAGNETOSPHERE BOUNDARY

Chapman and Ferraro formulated their theory for the shape of the magnetosphere boundary and the distortion of the geomagnetic field contained therein well over three decades ago (see Chapman, 1963, for a review), but the resulting free-boundary problem is sufficiently complex that even approximate solutions were not obtained until well into the satellite era. Although exact solutions in analytic form had been obtained earlier for related twodimensional problems suggested by the Chapman-Ferraro problem, much of the progress toward the solution of the actual three-dimensional problem is associated with the use of the approximation outlined on figure 13. The approximation that the magnetic field at the magnetosphere be proportional to the tangential component of the Earth's dipole field was first proposed to be used for the entire boundary by Beard (1960), although Ferraro (1952) had previously used the same approximation in a more limited sense to provide the basis for a brief discussion of the shape of the equatorial trace of the magnetosphere boundary. Through combination with the other condition that $\mathrm{H}^{2} / 8 \pi=p_{s t} \cos ^{2} \psi$ at the boundary and the expression

$$
\underset{\sim}{H}=-\left(\mathrm{a}_{\mathrm{e}}{ }^{3} \mathrm{H}_{\mathrm{eq}} / \mathrm{r}^{3}\right)(\hat{\theta} \sin \theta+\hat{\mathrm{r}} 2 \cos \theta)
$$

for the magnetic dipole field of the Earth, in which $a_{e}=6.37 \times 10^{8} \mathrm{~cm}$ is the radius of the Earth and $H_{e q}=0.312$ gauss is the intensity of the geomagnetic 
1. REPLACE $H_{n}=0$ AT BOUNDARY BY APPROXIMATION THAT $H=2 f\left(H_{d i}\right)_{t}$ WHERE $\left(H_{d i}\right)_{t}$ IS TANGENTIAL COMPONENT OF THE GEOMAGNETIC DIPOLE FIELD AT THE MAGNETOSPHERE BOUNDARY

2. COMBINE WITH $H^{2} / 8 \pi=K P_{\infty} V_{\infty}^{2} \cos ^{2} \psi$ TO OBTAIN

$$
\begin{gathered}
{\left[\frac{1+3 \cos ^{2} \theta}{R^{6}}\left(\frac{1}{R \sin \theta} \frac{\partial R}{\partial \phi}\right)^{2}+\frac{1}{R^{6}}\left(\sin \theta+\frac{2 \cos \theta}{R} \frac{\partial R}{\partial \phi}\right)^{2}\right]=} \\
\left(\sin \phi \sin \theta-\frac{\sin \phi \cos \theta}{R} \frac{\partial R}{\partial \theta}-\frac{\cos \phi}{R \sin \theta} \frac{\partial R}{\partial \phi}\right)^{2}
\end{gathered}
$$

WHERE $R=r / D$, AND $D=a_{e}\left(f^{2} H_{e q}^{2} / 2 \pi K P_{\infty} v_{\infty}^{2}\right)^{1 / 6}$

3. IN EQUATORIAL PLANE, $\theta=\pi / 2, \partial \mathrm{R} / \partial \theta=0$

$$
\frac{d R}{d \phi}=R\left(\frac{R^{6} \sin \phi \cos \phi+\sqrt{R^{6}-1}}{R^{6} \cos ^{2} \phi-1}\right) \text { FOR } \pi / 2 \leq \phi \leq 3 \pi / 2
$$

4. IN PRINCIPAL MERIDIAN PLANE, $\phi= \pm \pi / 2, \partial R / \partial \phi=0$

$$
\frac{d R}{d \theta}=(R \tan \theta) \frac{R^{3} \mp 1}{R^{3} \pm 2} \text { OR, SOLVING, } R \cos \theta=\frac{\bar{K} R^{3}}{R^{3} \mp 1}
$$

Figure 13. - Approximate solution of Chapman-Ferraro problem; dipole axis perpendicular to solar wind direction.

field at the magnetic equator, the partial differential equation shown on figure 13 may be derived for the case in which the dipole axis is perpendicular to the direction of the incident solar wind. This equation, which was first given by Davis and Beard (1962), is expressed in terms of a spherical coordinate system $r, \theta, \varphi$ with origin at the center of the Earth, polar axis alined with the geomagnetic axis, and the angle $\theta$ measured similar to colatitude with respect to the geomagnetic pole situated geographically at $78.6^{\circ}$ north latitude and $70.1^{\circ}$ west longitude near Thule, Greenland. The angle $\varphi$ is measured with respect to a line through the origin that is normal to both the dipole axis and the free-stream direction, and is equal to $\pi / 2$ when directed in the upstream direction, and $3 \pi / 2$ when directed downstream.

Considerable simplification occurs if attention is confined to either the geomagnetic equatorial plane or the principal meridian plane defined by the dipole axis and the direction of the incident solar wind. In each case, the partial differential equation reduces by reason of symmetry to an ordinary differential equation as indicated on figure 13. The equation for the equatorial trace of the magnetosphere boundary was integrated numerically by Beard (1960), and again somewhat more accurately by Spreiter and Briggs $(1961,1962)$. The equation for the principal meridian trace was derived by Beard (1960) who used the solution $R=1$ to show the upstream portion of the curve to be circular. Spreiter and Briggs (1961, 1962) subsequently considered the entire family of integral curves illustrated in figure 14 that are indicated by the complete solution written at the bottom of figure 13, in which $\bar{K}$ is an arbitrary constant of integration. They showed that only one possibility exists for a continuously connected boundary that remains at a finite and nonvanishing distance from the origin in the upstream direction and does not violate the condition that $\cos \psi \geq 0$. It is composed of part of the circular integral curve $R=1$ defined $b \bar{y} \bar{K}=0$ in the left plot joined onto part of the integral curve on the right plot defined by $\bar{K}=3 / 2^{2 / 3}=1.890$. 

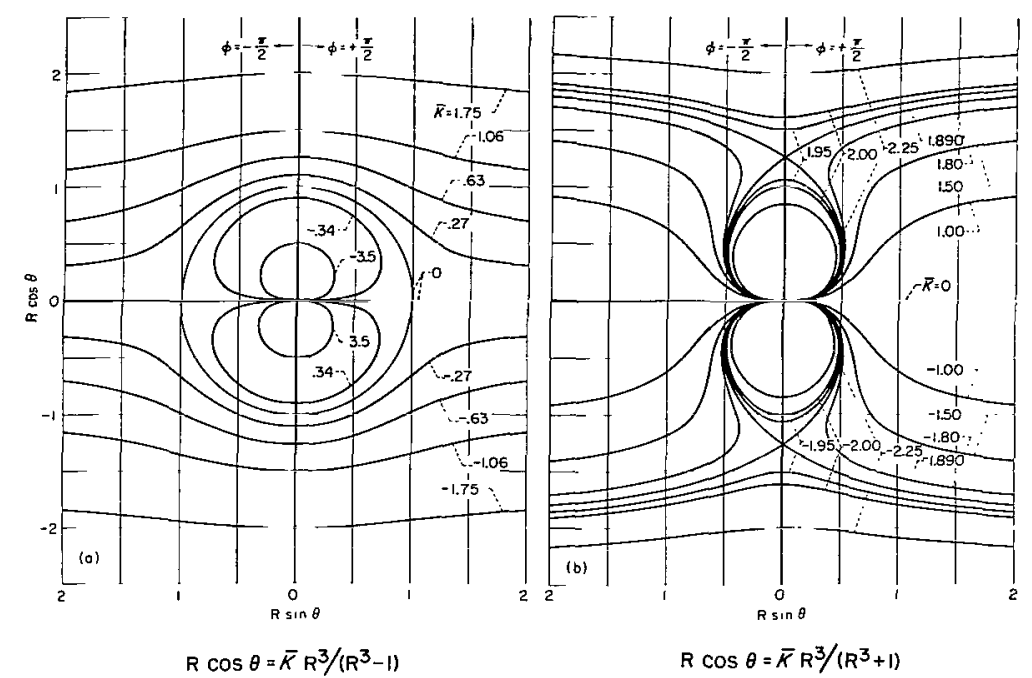

$\mathrm{R} \cos \theta=\bar{k} \mathrm{R}^{3} /\left(\mathrm{R}^{3}+1\right)$

Figure 14.- Integral curves in the principal meridian plane; dipole axis perpendicular to solar wind direction.

The resulting traces of the magnetosphere boundary in these two planes are shown in figure 15, together with a plot providing the geocentric distance $D$ to the magnetosphere nose defined in figure 13. The latter shows, for instance, that $D$ is about 10 Earth radii when the solar wind has a density of 10 protons $/ \mathrm{cm}^{3}$ and a velocity of $300 \mathrm{~km} / \mathrm{sec}$.

Coordinates for the remainder of the magnetosphere boundary were obtained subsequently by Briggs and Spreiter (1963), who solved numerically the partial differential equation shown in figure 13. The results are illustrated in figure 16. The pronounced dent in the boundary in the vicinity of the principal meridian plane is associated with the neutral point at which the magnetic field just inside the magnetosphere boundary vanishes in the idealized theory.

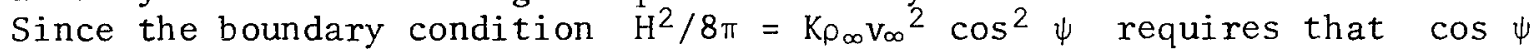
must vanish at such a point, it follows that the magnetosphere boundary must locally be parallel to the free-stream direction of the solar wind. This

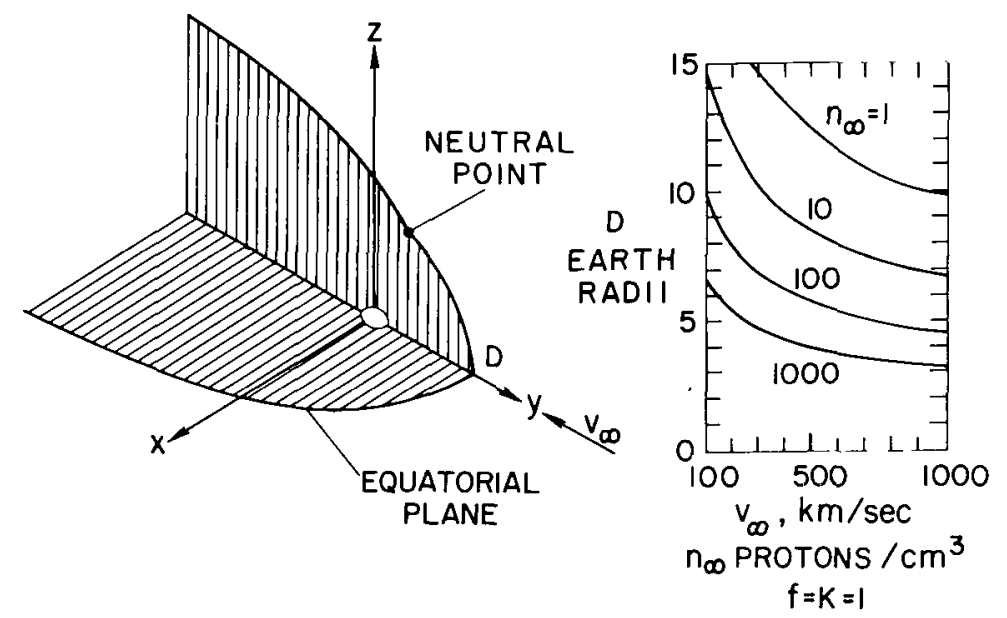

Figure 15.- Traces of magnetosphere boundary in magnetic equatorial plane and meridian plane containing dipole axis and solar wind velocity vector; $\lambda=0$. 


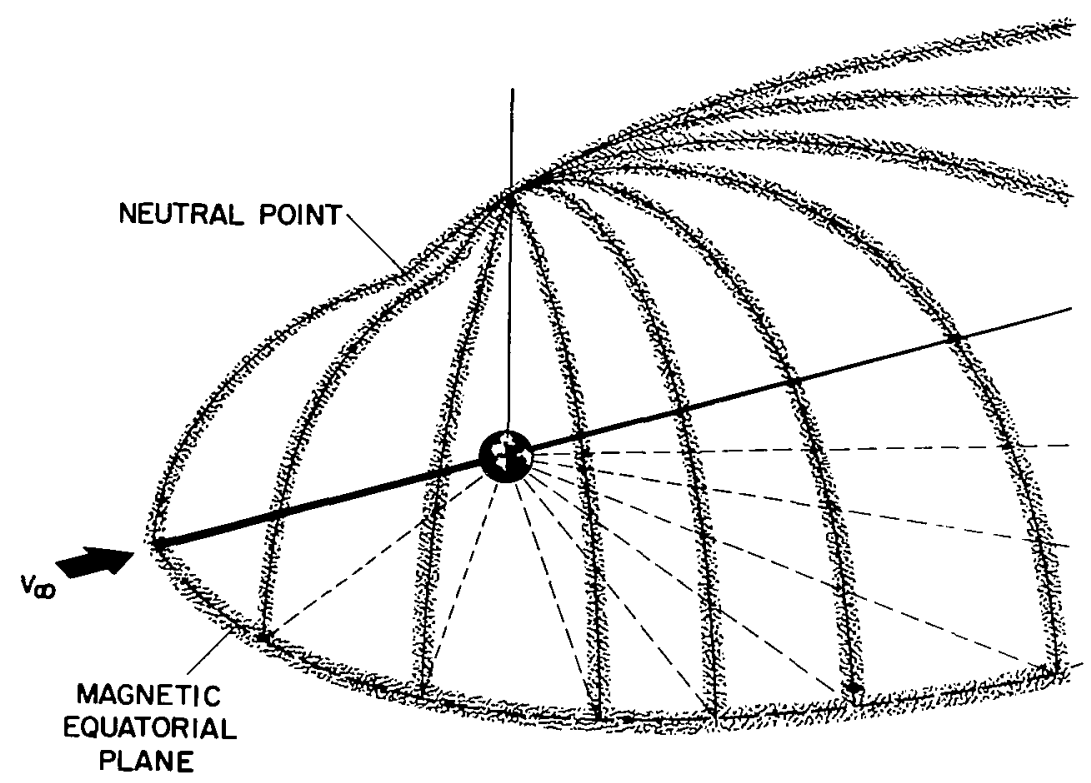

Figure 16. - The boundary of the geomagnetic field; $\lambda=0$.

point has further significance because there is only one field line in each hemisphere that extends from the Earth to the magnetosphere boundary in the idealized theory, and it joins the magnetosphere boundary at the neutral point. Further, all field lines in the magnetosphere boundary diverge from one neutral point and converge to the other.

The above discussion has been confined to the case in which the dipole axis is normal to the direction of the solar wind, or, equivalently, in which the angle $\lambda$ between the direction of the solar wind and the geomagnetic equatorial plane is zero. Because of the $23.5^{\circ}$ tilt of the Earth's geographic or spin axis with respect to the normal to the plane of the ecliptic, and the
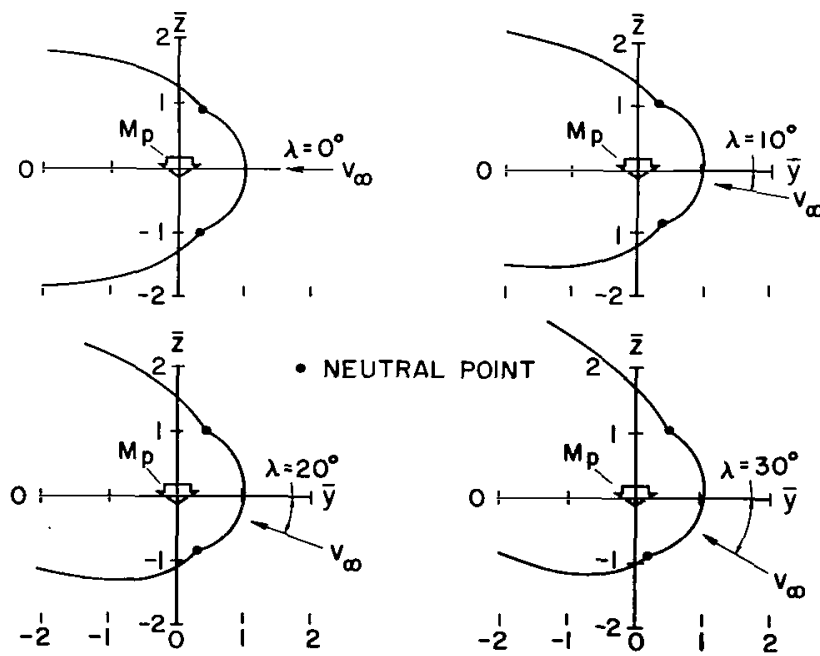

Figure 17.- Meridian traces of magnetosphere boundary for various angles between dipole axis and direction of solar wind. $11.6^{\circ}$ angle between the Earth's geographic and geomagnetic axes, the angle $\lambda$, however, is not always sma11. It is of interest, therefore, to determine the resulting effect on the shape of the magnetosphere boundary. The on $1 y$ theoretical results that are available on this topic are those for the principal meridian plane given by Spreiter and Briggs (1961, 1962).

Figure 17 presents the results of their analysis for several relative orientations of the dipole axis and the solar wind direction. They are determined by joining integral curves defined by the solution $R \cos (\theta-\lambda S) \mp\left(1 / R^{2}\right) \cos \theta=\bar{K}$ of 
the differential equation

$$
\left[R^{3} \cos (\theta-\lambda S) \pm 2 \cos \theta\right] d R / d \theta=R^{4} \sin (\theta-\lambda S) \mp R \sin \theta
$$

in a manner similar to that described for $\lambda=0$. The symbol $\overline{\mathrm{K}}$ in these expressions is again an arbitrary constant of integration and $S=\sin \varphi=\sin ( \pm \pi / 2)= \pm 1$.

The results shown in figure 17 indicate that the magnetosphere boundary, as viewed in geomagnetic coordinates, is influenced substantially by the direction of the solar wind. It is important to recognize that the effect is largely that of rotation into alinement with the direction of the solar wind. This point is illustrated in figure 18 by plotting essentially the same results as in figure 17 in terms of a coordinate system alined with the direction of the solar wind. They are also organized in a different way by

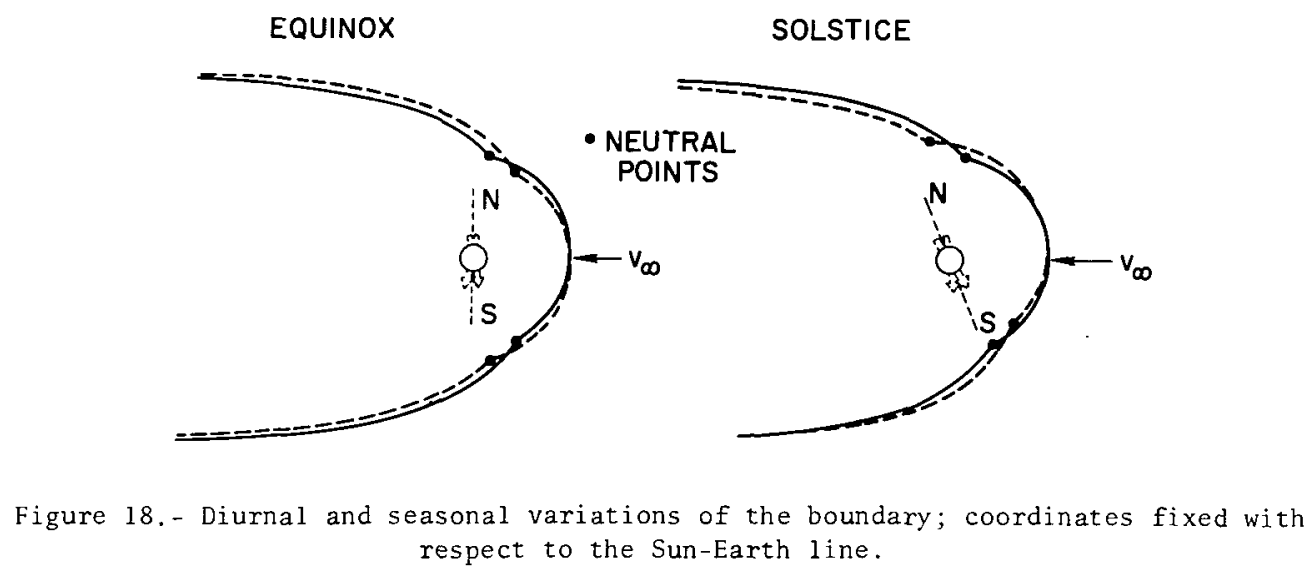

presenting together the results for the extremes of the diurnal variation at two significant times of the year, the equinox and the solstice, as indicated. It may be seen that the difference between the results is small, and that the only feature that is affected to any significant degree is the location of the neutral points. These results also indicate that the large open tail always extends exactly in the downstream direction, as it must in the exact solution of the Chapman-Ferraro problem. Also in accordance with the exact solution is the result that the asymptotic width of the magnetosphere tail in the principal meridian plane, for any given $\lambda$, is always exactly twice the distance between the two neutral points projected onto the normal to the direction of the free stream.

An evaluation of the accuracy of the results determined through use of the approximation outlined in figure 13 can be gained by comparison with the higher order approximations to the exact Chapman-Ferraro problem given by Midgley and Davis (1963) and Mead and Beard (1964) for the shape of the entire magnetosphere boundary for $\lambda=0$. Such a comparison is presented in figure 19 of the results for the equatorial and principal meridian planes. All three sets of results may be seen to be in reasonably good agreement, although the higher approximations tend to indicate a slightly larger flaring of the equatorial trace of the magnetosphere tail than indicated by the simpler 

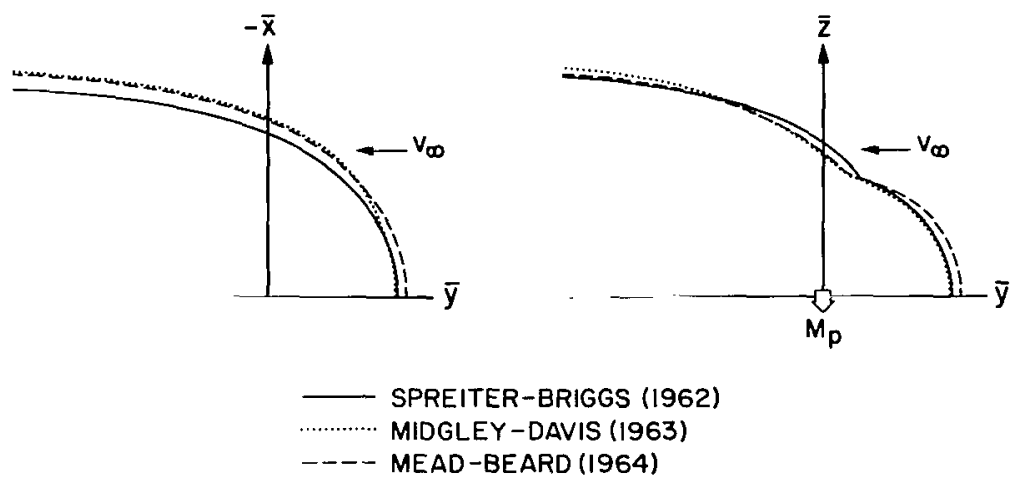

Figure 19.- Comparison with higher order approximations; $\lambda=0$.

calculations of Ferraro (1952), Beard (1960), and Spreiter and Briggs (1961, 1962). A similar trend has also been noted in some comparisons with observational data. It is difficult to be certain that this is the proper explanation, however, because, as will be illustrated later herein, the Newtonian pressure formula in the Chapman-Ferraro theory tends to underestimate the pressure exerted by the solar wind on the flanks and tail of the magnetosphere.

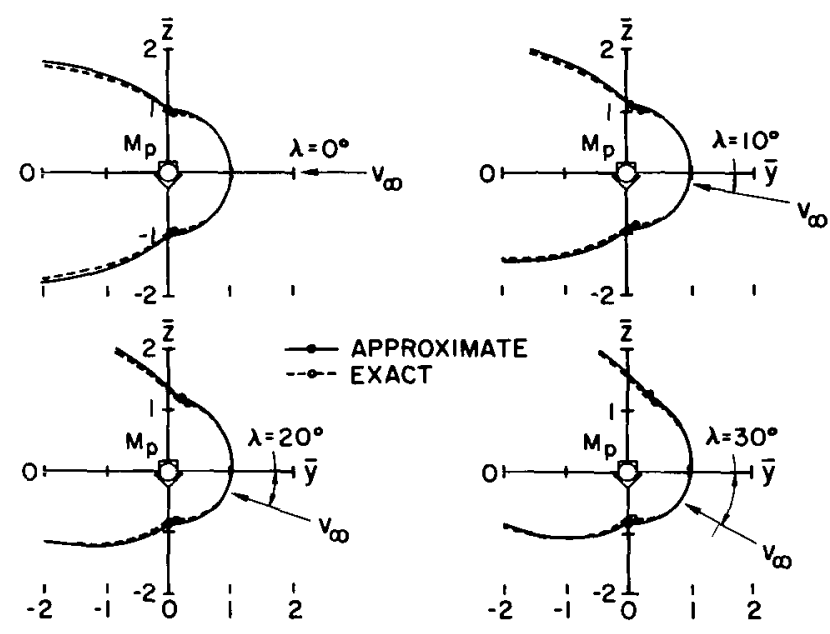

Figure 20.- Comparison of exact and approximate solutions for analogous two-dimensional problem.
Although no higher order approximations have yet been determined with which they may be compared, it is not anticipated that the accuracy of the approximate results for the magnetosphere boundary shown in figure 17 deteriorates particularly as $\lambda$ departs from zero. Support for this belief is provided by the comparisons shown on figure 20 between the exact solutions given by Zhigulev and Romishevskii (1960), Dungey (1961), and Hurley (1961) and the approximate solution given by Spreiter and Briggs (1961, 1962) for the corresponding problem of the interaction of the solar wind and a two-dimensional magnetic dipole. The latter has been determined in a way that is entirely analogous to that outlined on figure 13, except that the magnetic dipole field is now given by $\underset{\tilde{1}}{\mathrm{H}}=-\left(\mathrm{a}_{\mathrm{e}}{ }^{2} \mathrm{H}_{\mathrm{eq}} / \mathrm{r}^{2}\right)(\hat{\theta} \sin \theta+\hat{r} \cos \theta)$ and the length $D$ used to nondimensiona ${ }^{i z e}$ the coordinates of the magnetosphere boundary is changed as a result to $D^{2}=a_{e}\left(f^{2} H_{e}^{2} / 2 \pi K \rho_{\infty} v_{\infty}^{2}\right)^{1 / 4}$. Since the purpose of this comparison is to illustrate the degree to which the shape, but not particularly the size, of the magnetosphere can be determined by using the approximation $H=2 f\left(H_{d i}\right)_{t}$, the coefficient $f$ has been set to 0.913 in order to achieve a good fit with the exact solution at one point near the nose of the magnetosphere. If $f$ had been arbitrarily equated to unity, as it is usually done in the three-dimensional applications, the only change is that all coordinates of the magnetosphere boundary would be increased by the square root of $1 / 0.913$, or by about 4.5 percent. 
THE FLOW AROUND THE MAGNETOSPHERE

The preceding discussion has indicated how the exterior magnetosphere flow problem can be reduced without undue loss of accuracy to a purely gasdynamic problem of supersonic flow past a given blunt body, the shape of which is determined by solving the standard Chapman-Ferraro problem. Although this represents an enormous simplification, difficulties associated with the nonlinear and mixed elliptic-hyperbolic character of the governing partial differential equations are sufficiently great that it remains necessary at the present time to approximate the magnetosphere boundary with an axisymmetric shape. In the results to be reviewed here, the shape selected is that obtained by rotating the equatorial trace of the boundary given by the approximate solution of Spreiter and Briggs (1961, 1962) illustrated in figures 15 and 16. Inspection of the results shown in figures 15, 16, 18, and 19 shows that this is a reasonable approximation, except possibly where the boundary is dented inward around the neutral points. Even this exception may be of less importance than might be supposed, because, as will be shown later, these dents result from use of the Newtonian pressure approximation, and would disappear in any internally consistent analysis based on continuum fluid concepts.

The gasdynamic equations used to calculate the flow around the magnetosphere and the cylindrical coordinate system in which the results are presented are summarized in figure 21. The equations are just those indicated on

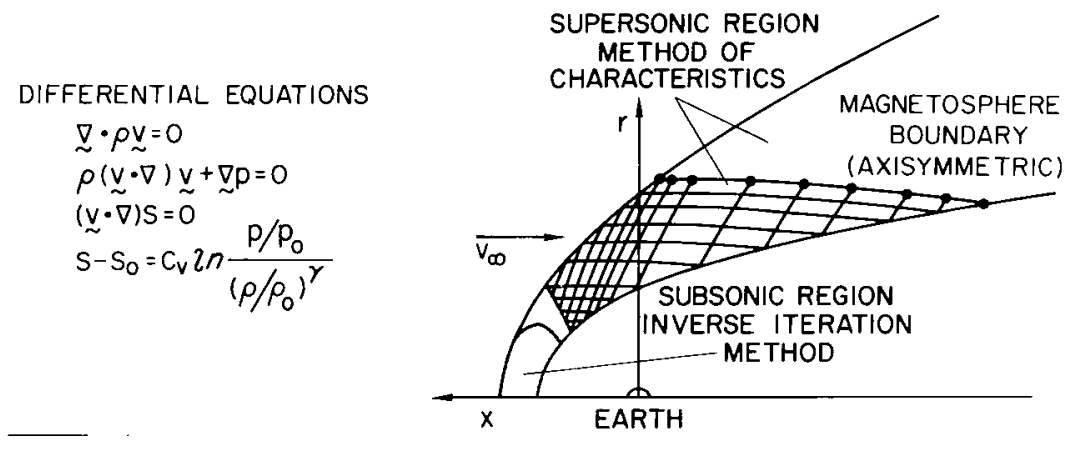

$$
\begin{aligned}
& \text { CONSERVATION EQUATIONS } \\
& {\left[\rho v_{n}\right]=0} \\
& {\left[\rho v_{n} \underset{v}{v}+\rho \hat{n}\right]=0} \\
& {\left[\rho v_{n}\left(h^{2}+v^{2} / 2\right)\right]=0}
\end{aligned}
$$

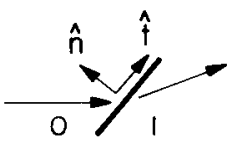

WHERE $[Q]=Q_{1}-Q_{O}$

Figure 21.- Gasdynamic equations for steady flow of a dissipationless perfect gas.

figure 11 after dropping the crossed out terms and introducing the assumption of axisymmetric flow. Because of the technological importance of this problem in the design of reentry vehicles, as well as intrinsic aerodynamic and mathematical interest, a considerable effort has been expended on this problem for more than a decade, and a variety of methods are now available for its numerical solution. The calculations of Spreiter, Summers, and Alksne (1966) to be reviewed here have been accomplished with the procedures described by Inouye and Lomax (1962) founded on the basic method of Van Dyke (1958) and Van Dyke and Gordon (1959) as modified by Fuller (1961). As illustrated in figure 21, 
the method used for the subsonic region near the magnetosphere nose and the immediately adjoining portion of the supersonic region is an indirect one in which the location of the bow shock wave and the free-stream conditions are assumed known and the associated flow field and body shape are found as part of the solution. The desired solution for the specified body, the magnetosphere boundary in the present application, is then found by iteration following judicious selection of the initial trial shape for the bow wave based on experience with a vast number of cases of aerodynamic interest. A detailed discussion of mathematical aspects, such as convergence, stability, and accuracy of the entire method, has been given by Lomax and Inouye (1964). The solution for the remainder of the supersonic region is accomplished as indicated by the method of characteristics described explicitly for this application by Inouye and Lomax (1962).

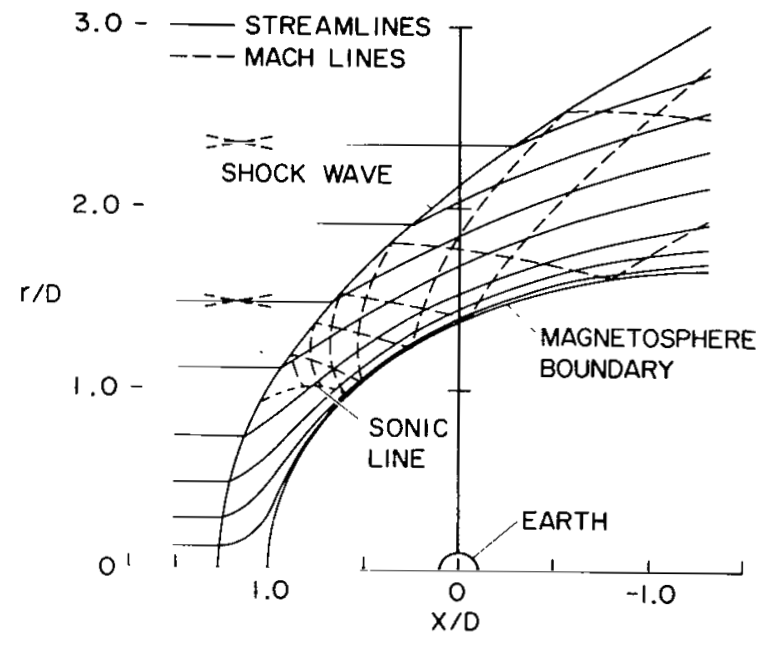

Figure $2 \angle$, - Streamlines and wave patterns for super sonic flow past the magnetosphere; $M_{\infty}=8$, $y=5 / 3$.
Figure 22 shows a plot of the results obtained in this way for the magnetosphere boundary and the bow shock wave, together with several streamlines and characteristic or Mach lines of the flow for a representative set of values for the free-stream Mach number $M_{\infty}$ and ratio of specific heats $\gamma$. The Mach lines, which correspond to standing compression or expansion waves of infinitesimal amplitude, cross the streamlines at such angles that the local velocity component normal to the wave is exactly equal to the local sound speed. Mach lines thus exist only where the flow is supersonic; they are absent from the vicinity of the magnetosphere nose because the flow there is subsonic.

Contour maps showing lines of constant density, velocity, and temperature, each normalized by dividing by the corresponding quantity in the incident stream, are presented in figure 23. As indicated, a single set of contours,
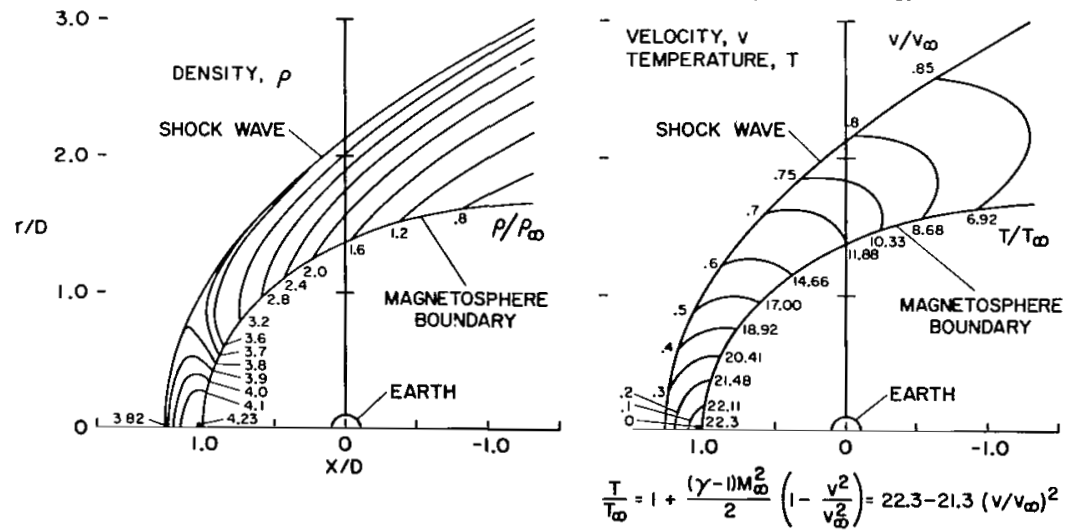

Figure 23. - Density, velocity, and temperature fields for supersonic flow past the magnetosphere; $M_{\infty}=8, Y=5 / 3$. 
although with different labels, serves for both the velocity and the temperature. These results show that the density ratio $\rho / \rho_{\infty}$ remains near the maximum value $(\gamma+1) /(\gamma-1)=4$ for a strong shock wave in a gas with $\gamma=5 / 3$ along nearly the entire length of the portion of the bow wave shown. The gas undergoes a small additional compression as it approaches the stagnation point at the magnetosphere nose and then expands to less than free-stream density as it flows around the flanks of the magnetosphere. The velocity remains less than in the free stream, however, throughout the same region. of particular interest is the tremendous increase in temperature of the solar wind as it passes through the bow wave. If, for example, the temperature of the incident solar wind is $10^{5} \mathrm{~K}$, the temperature at the stagnation point is indicated to be $2.23 \times 10^{6} \mathrm{~K}$. Such a value is of the same order of magnitude as the temperature possessed by the gas in the solar corona before it is accelerated to the high velocities characteristic of the solar wind in the vicinity of the Earth's orbit. This result serves to remind us that the energy content of the solar wind is, to a large extent, dependent on the temperature of the corona.

As noted in figure 11, the deformation of the interplanetary magnetic field by the flow around the magnetosphere can be accomplished directly, once the flow is determined, either by direct integration of $\underset{\sim}{\operatorname{cur}} \underset{\sim}{1}(\underset{\sim}{H} \times \underset{\sim}{\mathrm{v}})=0$, div $\underset{\sim}{H}=0$, and $\left[\mathrm{H}_{\mathrm{n}}\right]=0$ or by considering the field lines to drift with the fluid. In general, the field lines are spatial curves, but simplicity may be achieved at the expense of completeness by restricting attention to the plane through the center of the Earth that contains the velocity and magnetic field vectors in the incident stream. Results of two such calculations are shown in figure 24 for the same conditions as in figures 22 and 23 , namely, $M_{\infty}=8$ and $\gamma=5 / 3$. The field lines are as given previously by Spreiter, summers, and Alksne (1966), and the contour lines providing the intensity of the field in the plot on the left for $90^{\circ}$ angle between the directions of the flow and field in the incident stream are as given by Alksne (1967). The contour lines in the plot on the right for $45^{\circ}$ angle between these two directions have not been presented before, but have been calculated in exactly the same way as

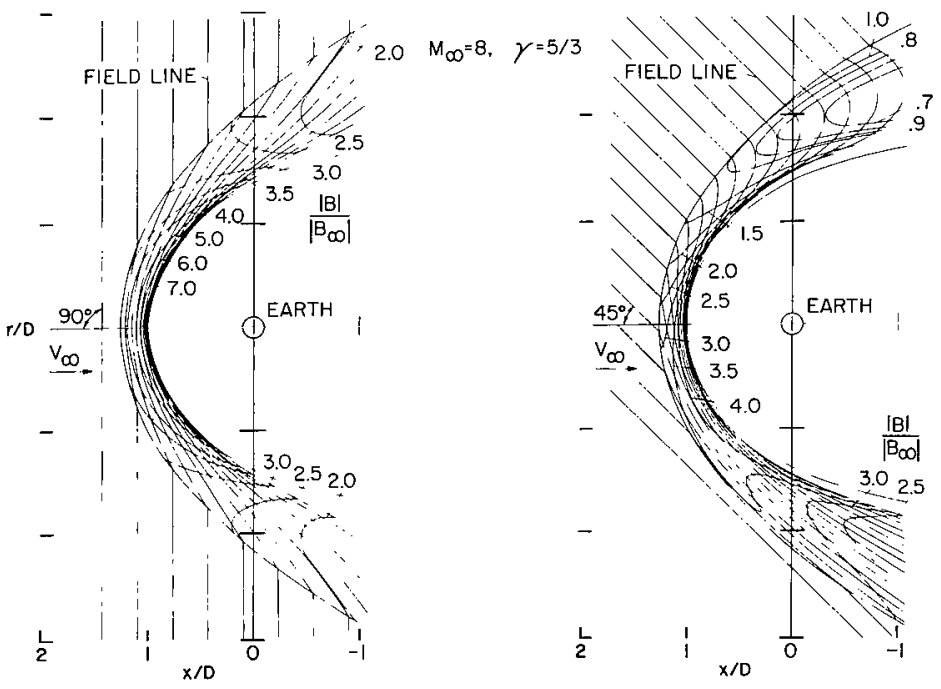

Figure 24.- Magnetic field in plane of free-stream velocity and magnetic field vectors for supersonic flow past the magnetosphere; $M_{\infty}=8, Y=5 / 3$. 
those given by Alksne (1967) for $30^{\circ}$ and $60^{\circ}$. The corresponding results for $0^{\circ}$ are not shown here, but can be obtained readily from the gasdynamic results, since it can be shown that the magnetic field is parallel to the flow and has such intensity that the ratio $H / H_{\infty}$ is equal to $\rho v / \rho_{\infty} v_{\infty}$ at all points.

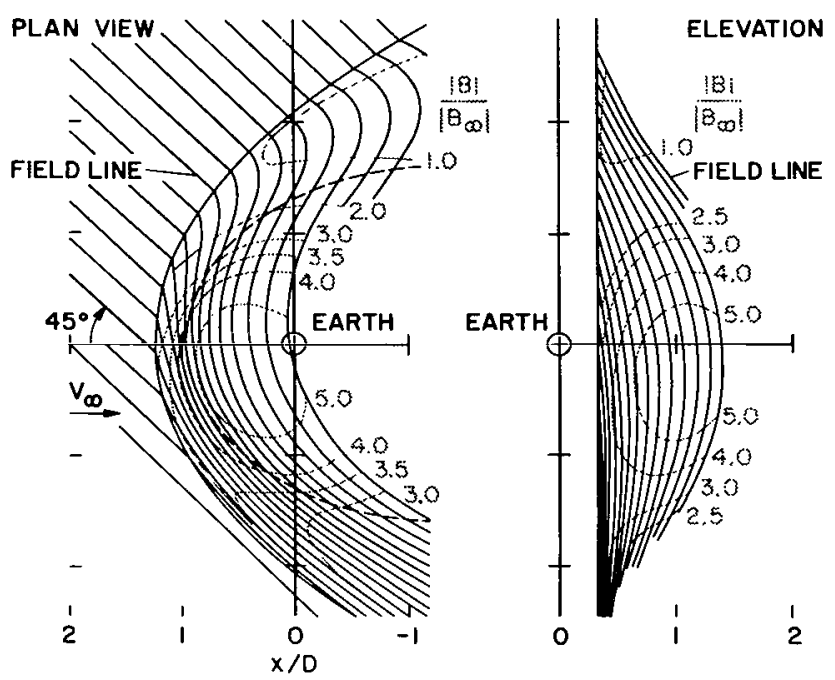

Figure 25. - Magnetic field for supersonic flow past the magnetosphere; $M_{\infty}=8, \gamma=5 / 3$.
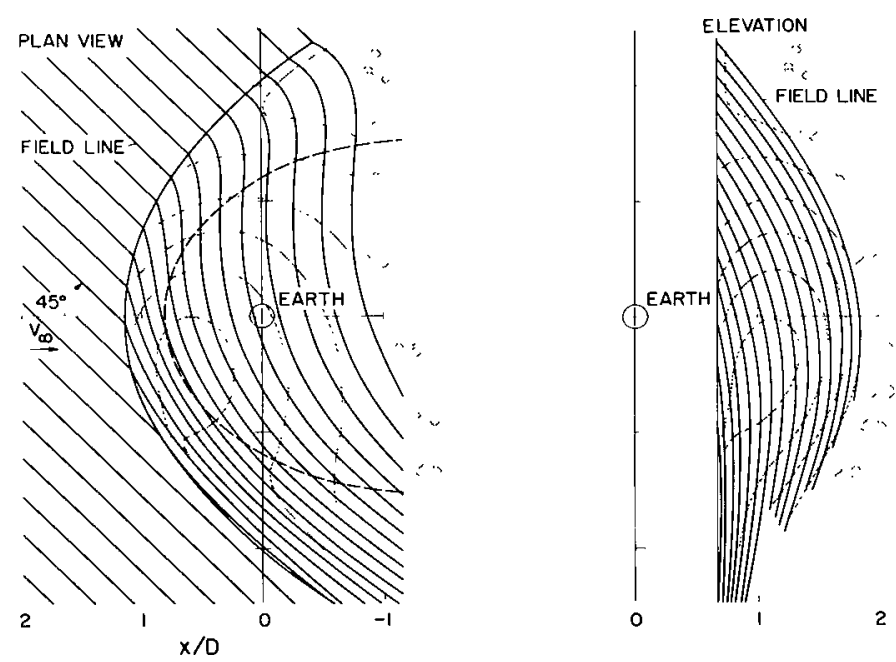

Figure 26.- Magnetic field for supersonic flow past the magnetosphere; $M_{\infty}=8, \gamma=5 / 3$.
Figure 25 shows the magnetic field configuration and intensity for the more complex case in which the set of field lines considered in the incident solar wind is contained in a plane parallel to the plane of symmetry but offset from it by a distance $D / 3$. The lines of intersection of the offset plane with the bow wave and the magnetosphere boundary are indicated by the solid and dashed lines, respectively. Corresponding results for the case in which the offset is $2 \mathrm{D} / 3$ are shown in figure 26. Since the field lines are no longer confined to a plane behind the bow wave, the resuits for each case are presented in the form of a pair of projections. It is of interest that the extreme crowding together of the field lines at the magnetosphere nose, so noticeable in figure 24 for the plane of symmetry, does not appear in figures 25 or 26 . Instead, the field lines appear as if they have slipped around the nose of the magnetosphere.

The results shown in figures 24 through 26 also indicate that the intensity of the magnetic field is usually a few times larger near the magnetosphere nose than in interplanetary space, and that it may be either larger or smaller than in the incident stream along the flanks of the magnetosphere. They also show that the direction of the field in the region between the bow wave and the magnetosphere is highly distorted by the flow, and bears little direct relation to the direction of the field in the incident solar wind.

The results shown in figures 22 through 26 are all for $M_{\infty}=8$ and $\gamma=5 / 3$. Since both the velocity and the temperature, and hence the speed of sound, of the solar wind vary substantially in the course of time, it is of 
interest to review some of the results given by Spreiter, Summers, and Alksne (1966) for other Mach numbers. Figure 27 shows plots of contours of constant density ratio $\rho / \rho_{\infty}$ for free-stream Mach numbers of 5 and 12 . Although differences are clearly evident, they remain sufficiently small to be of only secondary importance for most purposes. The corresponding results for the velocity and temperature are shown in figure 28 . Like the density, the velocity field is hardly affected by the change from Mach number 5 to 12 . The temperature fields for the two Mach numbers are substantially different, however, with the temperatures for Mach number 12 generally being about five times larger than for Mach number 5. Such strong effects on the temperature can be understood easily in terms of the expression for $T / T_{\infty}$ shown on figure 23 .
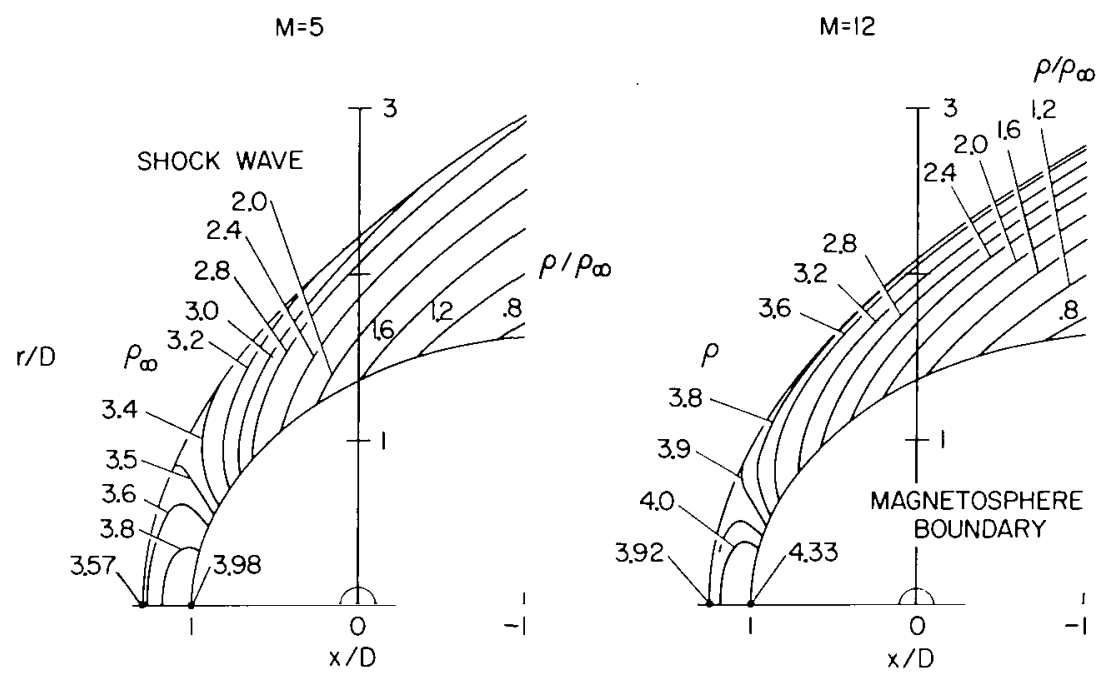

Figure 27.- Effect of Mach number on density field for flow past the magnetosphere; $\gamma=5 / 3$.
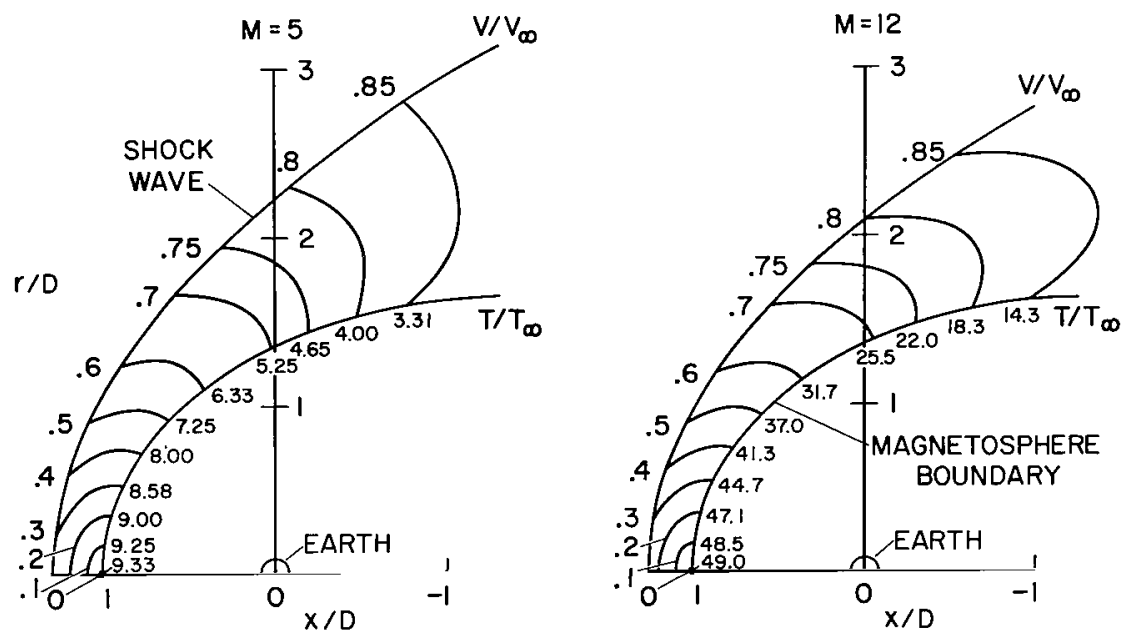

Figure 28.- Effect of Mach number on velocity and temperature fields for flow past the magnetosphere; $\gamma=5 / 3$. 
Although the results will not be displayed graphically here, the temperature contours can also be put into a form relatively independent of $M_{\infty}$ by normalizing the temperature by dividing by $\mathrm{v}_{\infty}{ }^{2}$ rather than $\mathrm{T}_{\infty}$. Optionally, we may consider the ratio $2 c_{v}\left(T-T_{\infty}\right) / v_{\infty}{ }^{2}$ of the change in internal energy $c_{v}\left(T-T_{\infty}\right)$ to the kinetic energy $v_{\infty} 2 / 2$, both per unit mass, or even better, perhaps, the ratio $2 \mathrm{c}_{\mathrm{p}}\left(\mathrm{T}-\mathrm{T}_{\infty}\right) / \mathrm{v}_{\infty}{ }^{2}$ of the change of the enthalpy $c_{p}\left(T-T_{\infty}\right)=\gamma c_{v}\left(T-T_{\infty}\right)$ to the kinetic energy. That this is so may be demonstrated by combining the expression $\left(T-T_{\infty}\right) / T_{\infty}=[(\gamma-1) / 2] M_{\infty}{ }^{2}\left(1-v^{2} / v_{\infty}{ }^{2}\right)$, the relation $h=c_{\mathrm{p}} \mathrm{T}$ defining the enthalpy per unit mass, and the relation $\mathrm{M}_{\infty}=\mathrm{v}_{\infty} /\left(2 \gamma \mathrm{RT}_{\infty}\right)^{1 / 2}$ for the free-stream Mach number of fully ionized hydrogen. The quantities $c_{p}$ and $c_{v}$ in these expressions represent the specific heats at constant pressure and volume. They are related to the gas constant $\mathrm{R}$ according to $\mathrm{R}=\mathrm{c}_{\mathrm{V}}(\gamma-1) / 2=\mathrm{c}_{\mathrm{p}}(\gamma-1) / 2 \gamma=8.315 \times 10^{7} \mathrm{ergs} / \mathrm{gm}^{\mathrm{O}} \mathrm{K}$. The resulting expression is $2 c_{p}\left(T-T_{\infty}\right) / v_{\infty}{ }^{2}=\left(1-v^{2} / v_{\infty}{ }^{2}\right)$. Since $v^{2} / v_{\infty}{ }^{2}$ tends to be independent of Mach number at large $M_{\infty}$, as illustrated in figure 28 for $M_{\infty}=5$ and 12 , and $T_{\infty}$ in the same region is very much smaller than $T$, it follows that the ratio $2 c_{p}\left(T-T_{\infty}\right) / v_{\infty}{ }^{2}$ also tends to be invariant with changes in Mach number at large $M_{\infty}$.

The conditions in the flow field are also affected by changes in the ratio of specific heats $\gamma$. Earlier calculations, such as those of Spreiter and Jones (1963), have frequently used $\gamma=2$, and Dryer and Heckman (1967) have recently used $\gamma=1.2$. Neither the theoretical reasons nor the observational data provide compeliing support, in our estimation, for the use of these values. We recommend, therefore, that the usual value for a monatomic gas, that is, $Y=5 / 3$, be used for the present. It is, nevertheless, of value to possess an understanding of the effects of substantial variations of both $M_{\infty}$ and $\gamma$ on the theoretical results. In order to accomplish this in a concise, yet still comprehensive, form, figure 29 from Spreiter, Summers, and Alksne (1966) has been included to summarize the effects of $M_{\infty}$ and $\gamma$ on the stand-

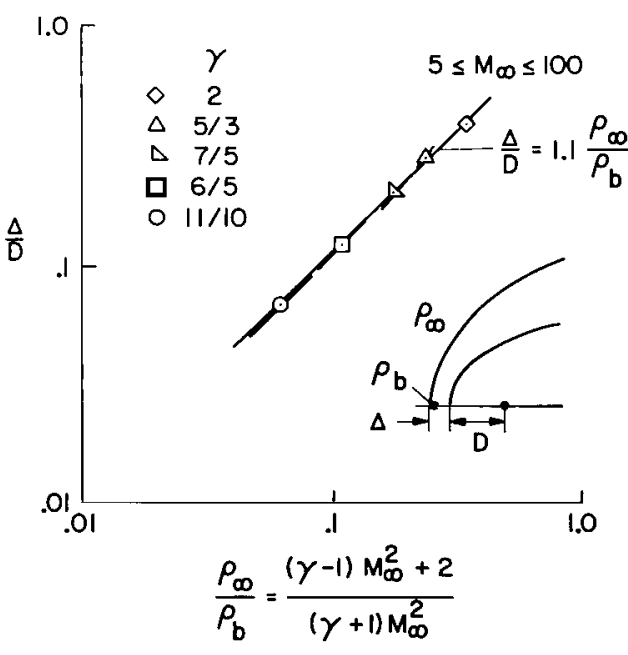

Figure 29. - Bow wave standoff distance for supersonic flow past the magnetosphere. off distance of the bow wave from the magnetosphere nose. These results, collected from a large number of calculations for a wide range of values for $M_{\infty}$ and $\gamma$, show that the ratio of the standoff distance $\Delta$ to the geocentric distance to the magnetosphere nose $D$ is very nearly proportional to the density ratio $\rho_{\infty} / \rho_{b}$ across the nose of the bow wave. The latter is, moreover, related to $M_{\infty}$ and $\gamma$ by the simple expression shown at the bottom of figure 29. For Mach numbers large compared with unity, it reduces to $\rho_{\infty} / \rho_{b}=(\gamma-1) /(\gamma+1)$, or simply $1 / 4$ for a gas with $\gamma=5 / 3$. 


\section{MICROSCOPIC INTERPRETATION OF CONTINUUM RESULTS}

In justifying the use of a continuum theory of fluid flow, be it a gasdynamic, magnetohydrodynamic, or more complicated description, to calculate the gross average features of the solar wind and its interaction with the geomagnetic field, appeal is usually made to possible interaction mechanisms involving electric and magnetic fields, and possibly to plasma instabilities, that operate over distances that are not only smaller than the ordinary mean free paths of the particles, but, more importantly, smaller than the scale of the flow feature being examined. When attention is turned from the gross features of the flow to the quantities actually measured on board spacecraft with, say, a plasma probe, the situation alters significantly because the instruments are much smaller than any of the suggested interaction distances. It thus appears necessary to return to a microscopic picture based on kinetic theory both to interpret certain features of the data and to carry the theoretical calculations to the point where the results are directly comparable with observations. A complete treatment of the interaction of the solar wind with the geomagnetic field based on kinetic theory is, however, an intractable, and probably unnecessarily complex, task at the present time.

One may, instead, follow the procedure employed by Spreiter, Alksne, and Abraham-Shrauner (1966) who combined results for the bulk properties of the flow provided by fluid models with relations for the velocity distribution provided by kinetic theory. The basic additional assumption is that the instantaneous microscopic velocity distribution of the ions is Maxwellian in a frame moving with the local bulk velocity of the flow. In ordinary gases, near standard conditions, the use of a Maxwellian distribution would hardly require comment because its applicability is essentially guaranteed by the rapid rate of collisions between particles. In the solar wind, the collision rate is very low and it is neither obvious nor necessarily correct that the velocity distribution is Maxwellian. Nevertheless, the Maxwellian distribution is useful at least as a rough guide, and may even be a reasonably good approximation to the conditions encountered much of the time in space. The use of a Maxwellian velocity distribution is, moreover, consistent with the use of continuum equations of magnetohydrodynamics and gasdynamics in the fluid models, and with current practice in analyzing and interpreting data from plasma probes on some of the recent spacecraft.

According to the Maxwellian velocity distribution, the number density dn of a species of particles of mass $m$ having velocities between $\underset{\sim}{w}$ and $\underset{\sim}{w}+d \underset{\sim}{w}$

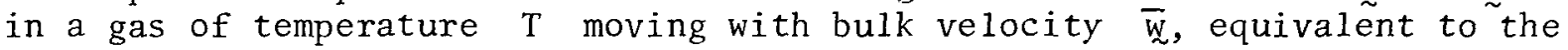
gasdynamic velocity $\underset{\sim}{v}$, is as given in the first expression on figure 30 , in which $\mathrm{k}=1.38 \times 10^{-16} \mathrm{erg}^{\circ} \mathrm{K}$ is Boltzmann's constant, $\mathrm{n}$ is the total number density of the species of particles independent of their velocity, and $u, v$, and $w$ are Cartesian components of $w$. We proceed to apply this expression to the ions, and note that $m=1.67 \times 10^{-24} \mathrm{gm}$ if they are hydrogen ions, or protons. The quantity $(2 \mathrm{kT} / \mathrm{m})^{1 / 2}$ has the dimensions of velocity, and is recognized to be the most probable particle velocity in a reference frame in which the bulk velocity $\bar{W}$ is zero, that is, in a reference frame moving with the local bulk velocity. It is proportional to, and less than, the speed of sound 
IN CARTESIAN COORDINATES $u, v, w$ IN VELOCITY SPACE

$$
d n=n\left(\frac{m}{2 k T}\right)^{3 / 2} \exp \left[\frac{-m(\underset{w}{w}-\bar{w})^{2}}{2 k T}\right] d u d v d w
$$

IN WHICH $\underset{w}{W}$ IS THE PARTICLE VELOCITY $\widetilde{W}$ IS THE BULK VELOCITY

IN SPHERICAL COORDINATES $r, \theta, \phi$ IN VELOCITY SPACE

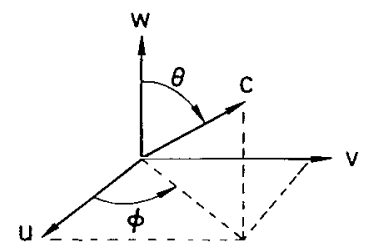

$$
\mathrm{d} n=n\left(\frac{m}{2 k T}\right)^{3 / 2} \exp \left[\frac{-m\left(c^{2}+\bar{w}^{2}-2 c \bar{w} \cos \theta\right)}{2 k T}\right] c^{2} d c d \Omega
$$

IN WHICH $c=\left(u^{2}+v^{2}+w^{2}\right)^{1 / 2}$

$\mathrm{d} \Omega=\sin \theta \mathrm{d} \theta \mathrm{d} \phi$ IS AN INFINITESIMAL SOLID ANGLE

OR, IN NONDIMENSIONAL FORM WITH

$C=c /(2 k T / m)^{1 / 2}, \quad \bar{W}=\bar{W} /(2 k T / m)^{1 / 2}$

$\frac{d n}{n}=\frac{c^{2}}{\pi^{3 / 2}} \exp \left[-\left(c^{2}+\bar{W}^{2}-2 c \bar{w} \cos \theta\right] d c d \Omega\right.$

Figure 30.- Equations of Maxwellian velocity distribution.

in accordance with the relations $(2 \mathrm{kT} / \mathrm{m})^{1 / 2}=(\mathrm{p} / \mathrm{\rho})^{1 / 2}=\mathrm{a} / \mathrm{\gamma}^{1 / 2}=0.774 \mathrm{a}$ which may be derived from the expression $a=(\gamma p / \rho)^{1 / 2}$ for the speed of sound by replacing the pressure $p$ by the combined pressure $2 \mathrm{nkT}$ of the ions and electrons, and the density $\rho$ by the product $m n$ of the mass and number density of the ions, the contribution of the electrons to the density being negligible.

It is useful, because of the axial symmetry of the Maxwellian velocity distribution about the direction of the bulk velocity vector, to introduce a spherical coordinate system in velocity space related to a rectangular Cartesian coordinate system in which the $w$ axis is alined with the bulk velocity vector $\bar{W}$ as illustrated in figure 30 . The expression for the Maxwellian velocity distribution can thus be rewritten in terms of the particle speed $c=\left(u^{2}+v^{2}+w^{2}\right)^{1 / 2}$ and the direction of its motion $\theta$ with respect to the direction of the bulk velocity vector $\underset{\sim}{\vec{W}}$ in the form shown in the second equation on figure 30. Introduction of the dimensionless quantities $C$ and $\bar{W}$ obtained by dividing $c$ and $\bar{W}$ by $(2 \mathrm{kT} / \mathrm{m})^{1 / 2}$ leads to the 1 ast equation shown on figure 30. This expression shows that the fraction $\mathrm{dn} / \mathrm{n} \mathrm{dC} \mathrm{d} \Omega$ of the particles per unit solid angle and unit dimensionless speed depends only on the dimensionless speed $C$ and direction $\theta$ with respect to that of the local bulk velocity of the particles being counted, and the ratio $\vec{W}$ of the local bulk speed to the most probable speed in a frame moving with the local bulk velocity. The quantity $\bar{W}$ is moreover directly related to the local Mach number $M$ of the flow since $\bar{W}=w /(2 \mathrm{kT} / \mathrm{m})^{1 / 2}=\gamma^{1 / 2} \overline{\mathrm{w}} / \mathrm{a}=1.29 \mathrm{M}$.

Plots of $\mathrm{dn} / \mathrm{n} \mathrm{dC} d \Omega$ as a function of $\theta$ for several representative values of $C$ and $\bar{W}$ are presented in figure 31 . Also included is a plot in the upper left part of this figure presenting the location of lines of constant $\bar{W}$ for the special case in which $M_{\infty}=8$ and $\gamma=5 / 3$, as in figures 22 through 26. The velocity distribution having the corresponding value for $\bar{W}$ is thus appropriate at any point along the contour lines. The velocity distributions themselves are general, however, and are applicable as well for other values for $M_{\infty}$ and $\gamma$, provided that they be applied at the location 


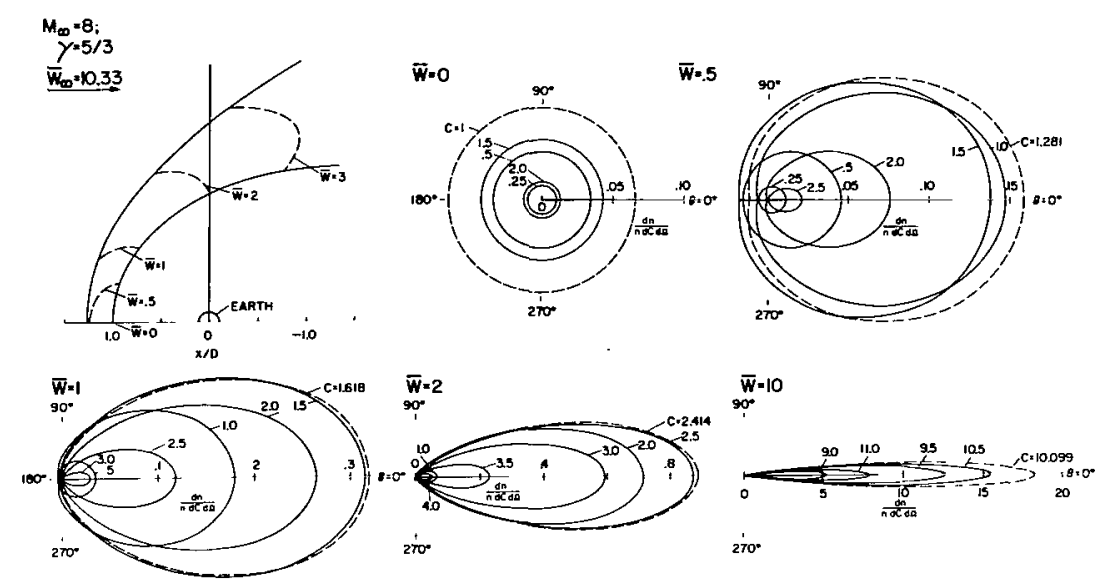

Figure 31.- Polar plots of Maxwellian proton velocity distributions corresponding to conditions in supersonic flow past the magnetosphere.

where the designated value for $\bar{W}$ prevails. The plots included in figure 31 display how the velocity distribution changes from being completely isotropic when $\bar{W}$ is zero, as at the magnetosphere nose, to being highly collimated when $\bar{W}=10$, as in the undisturbed solar wind when the free-stream Mach number $M_{\infty}=(3 / 5)^{1 / 2} \times 10=7.75$. For fixed $C$ and $\bar{W}, \mathrm{dn} / \mathrm{n} \mathrm{dC} d \Omega$ is a maximum when $\theta=0$ and a minimum when $\theta=\pi$, as can be seen by equating $\mathrm{d}(\mathrm{dn} / \mathrm{n} \mathrm{dC} \mathrm{d} \Omega) / \mathrm{d} \theta$ to zero and investigating the $\operatorname{sign}$ of $\mathrm{d}^{2}(\mathrm{dn} / \mathrm{n} \mathrm{dC} \mathrm{d} \Omega) / \mathrm{d} \theta^{2}$ for $\theta=\pi$ and $\theta=0$.

In figure 32 the velocity distribution $\mathrm{dn} / \mathrm{n} \mathrm{dC} \mathrm{d} \Omega$ is illustrated a second way, as a function of the dimensionless particle speed $C$ for fixed $\bar{W}$ and $\theta$. The minimum value for $\mathrm{dn} / \mathrm{n} d C \mathrm{~d} \Omega$ for fixed $\theta$ and $\bar{W}$ is zero, and
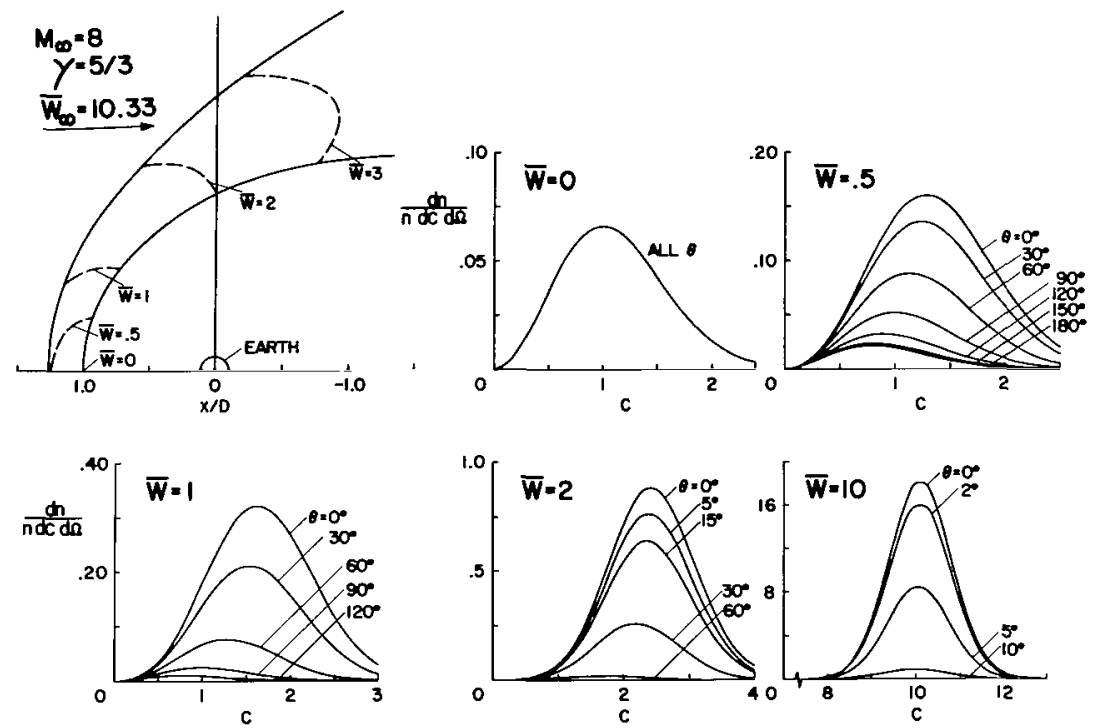

Figure 32.- Maxwellian proton velocity distributions corresponding to conditions in supersonic flow around the magnetosphere. 
occurs when $C$ is either zero or infinite. The maximum value can be found by equating $d(d n / n d C d \Omega) / d C$ to zero and solving for $C$. It is

$$
C=\left[\bar{W} \cos \theta+\left(\bar{W}^{2} \cos ^{2} \theta+4\right)^{1 / 2}\right] / 2
$$

For small $\overline{\mathrm{W}} \cos \theta, \mathrm{dn} / \mathrm{n} \mathrm{dC} \mathrm{d} \Omega$ is thus a maximum when $\mathrm{C}=1+(\overline{\mathrm{W}} \cos \theta) / 2$. For large $\bar{W} \cos \theta$, the maximum value occurs at either $C=\bar{W} \cos \theta$ or $C=-1 /(\bar{W} \cos \theta)$, depending on whether $\bar{W} \cos \theta$ is positive or negative. Since there is only one solution for $C$ for given $\bar{W}$ and $\theta, \mathrm{dn} / \mathrm{n} \mathrm{dC} \mathrm{d} \Omega$ decreases monotonically on both sides of the maximum, and approaches zero as $C$ approaches zero and infinity.

Combining the results of the two preceding paragraphs, we see that the absolute maximum value for $\mathrm{dn} / \mathrm{n} \mathrm{dC} \mathrm{d} \Omega$ occurs when $\theta$ is zero and $\mathrm{C}=\mathrm{C}_{\mathrm{M}}=\left[\overline{\mathrm{W}}+\left(\overline{\mathrm{W}}^{2}+4\right)^{1 / 2}\right] / 2$. Curves illustrating the variation of $\mathrm{dn} / \mathrm{n} \mathrm{dC} \mathrm{d} \Omega$ with $\theta$ for $C=C_{M}$ are included on figure 31 and indicated by dashed lines. Similarly, the smallest maximum value for $\mathrm{dn} / \mathrm{n} \mathrm{dC} d \Omega$ for fixed $\overline{\mathrm{W}}$ occurs when $\theta=\pi$ and $C=\left[-\bar{W}+\left(\bar{W}^{2}+4\right)^{1 / 2}\right] / 2$.

The preceding results can be applied to the flow around the magnetosphere by inserting into the equations shown on figure 30 the values for density, temperature, and velocity calculated using the fluid models. Figure 33 shows the results of a specific application for the case illustrated in figures 22 through 26, namely, $M_{\infty}=8$ and $\gamma=5 / 3$. The solar wind is considered to consist entirely of protons and electrons, and to flow in the undisturbed incident stream with a velocity $v_{\infty}=\bar{w}_{\infty}=4 \times 10^{7} \mathrm{~cm} / \mathrm{sec}$. The temperature $T_{\infty}$ in the free stream is thus $0.90 \times 10^{5} \mathrm{oK}$. Several kinds of results are included on this figure. First of all, there are lines representing the magnetosphere boundary and the bow shock wave, streamlines, and contour lines of constant speed and temperature, all of which correspond to those shown on figures 22 and 23. The latter are also contour lines of constant local Mach number $M$ and $\bar{W}$ as indicated on the figure. Small diagrams are presented at several points of intersection of the streamlines and the contour lines that

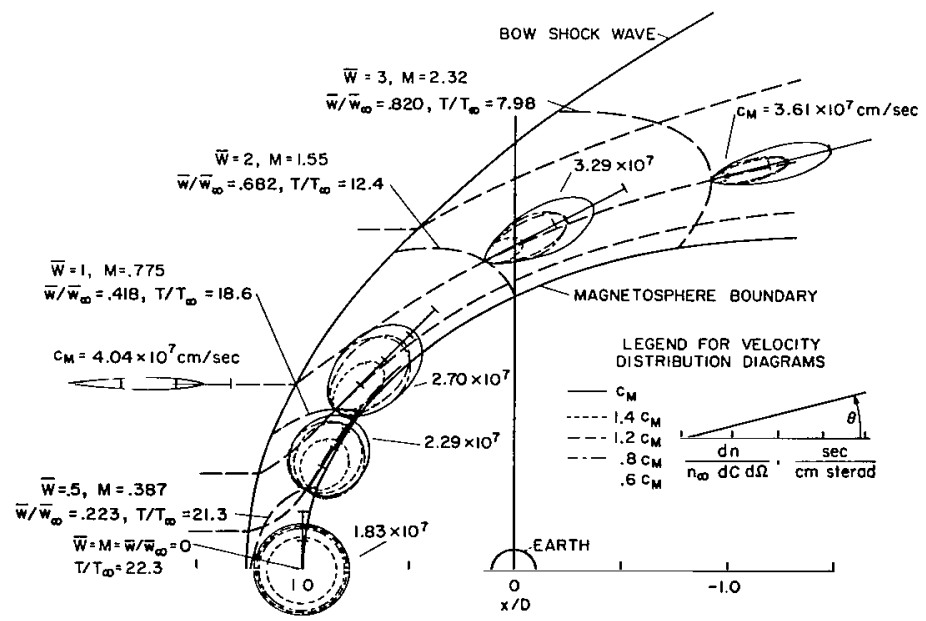

Figure 33.- Maxwellian proton velocity distributions at several points in the flow around the magnetosphere; $M_{\infty}=8, \gamma=5 / 3, w_{\infty}=400 \mathrm{~km} / \mathrm{sec}, T_{\infty}=90,0000 \mathrm{~K}, \bar{w}_{\infty}=10.33$. 
illustrate the velocity distribution of the protons. These diagrams are similar to those of figure 31 in that they show on a polar plot the variation of number density with direction of motion for particles of selected speeds. The speeds selected for each of the diagrams include $c=c_{M}=(2 \mathrm{kT} / \mathrm{m})^{1 / 2} \mathrm{C}_{M}$ so that the curve displaying the absolute maximum value for the number density is always shown. The values for $c$ for the other curves are simple fractions and multiples of $c_{M}$. The diagrams differ from those of figure 31 in that $\mathrm{dn} / \mathrm{n}_{\infty} \mathrm{dc} \mathrm{d} \Omega$ is plotted rather than $\mathrm{dn} / \mathrm{n} \mathrm{dC} d \Omega$ in order to illustrate better the relation between the velocity distributions at different points. They are also oriented on figure 33 so that $\theta=0$ in the direction of the local flow velocity. These plots illustrate how the velocity distribution is highly collimated in the incident solar wind, completely isotropic at the stagnation point at the magnetosphere nose, and changes back to that of a highly collimated stream along the flanks of the magnetosphere. Except for a limited region in the immediate vicinity of the nose, only a small fraction of the total number of particles at a given point move in the upstream direction $(\theta=\pi)$. Throughout the entire flow, however, the speed $c_{M}$ corresponding to the maximum number density at each of the stations tends to be relatively constant in spite of substantial changes in the velocity and temperature of the gas.

\section{EVALUATION OF THEORY AND COMPARISON WITH LABORATORY OBSERVATIONS}

It is informative and important to examine the foregoing theoretical results for internal consistency and to compare them with observations made in the laboratory, as well as in space. One of the first questions that often arises in such a discussion is how well does the simple Newtonian pressure formula $\mathrm{p}=\mathrm{p}_{\mathrm{s}} \cos ^{2} \psi$ provide for the variation of pressure along the magnetosphere boundary. This can be answered partially in the present applica-

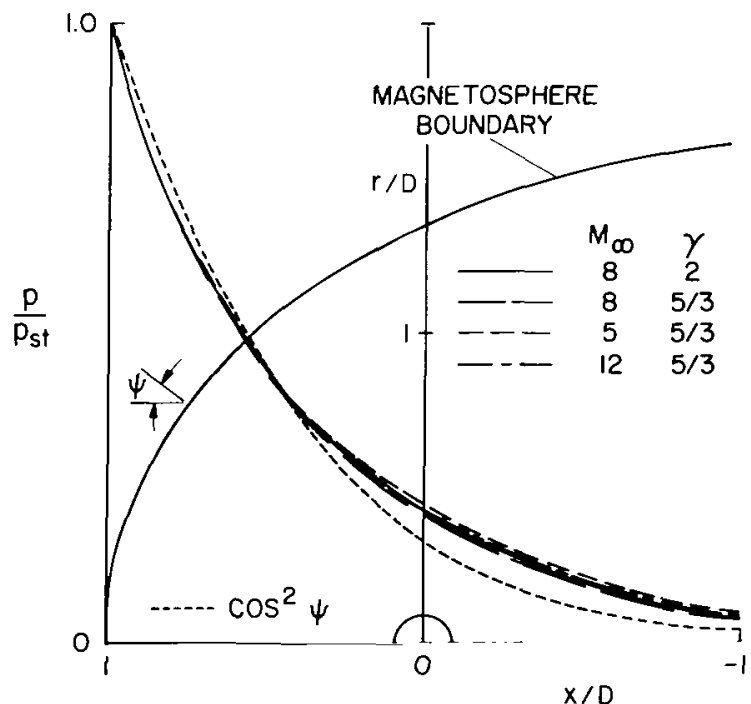

Figure 34.- Comparison of exact and approximate pressure distributions on magnetosphere boundary. tions by comparing the pressure so determined with that indicated by the detailed gasdynamic calculations. The results of such a comparison, origina1ly presented by Spreiter, Summers, and Alksne (1966), are shown in figure 34 for several values for $M_{\infty}$ and $\gamma$. It can be seen that the simple Newtonian expression does indeed provide a generally good approximation over most of the magnetosphere boundary of interest in the present studies. The agreement is particularly good over the portion of the magnetosphere along which the flow is subsonic. No significant changes are to be expected, therefore, in the shape of the nose portion of the bow wave due to the use of a more accurate and necessarily more complicated calculation of the pressure. The Newtonian pressure formula does, 
however, underestimate the pressure somewhat along the flanks of the magnetosphere, indicating that a revised calculation using the gasdynamic pressure distribution would lead to a slightly slimmer magnetosphere. The resulting change in the magnetosphere shape would be expected to be small, however, because of the magnetic pressure against which the gas pressure is balanced according to the boundary condition $\mathrm{H}^{2} / 8 \pi=p_{s t} \cos ^{2} \psi$ is approximately proportional to the inverse sixth power of the distance from the center of the Earth. Whether a more accurate solution of the complete problem would result in a magnetosphere that is slightly thinner or slightly more flared remains uncertain, however, because of the counterbalancing difference between the exact and approximate solution of the Chapman-Ferraro problem illustrated in figure 19. A point of equal interest displayed by these results is the lack of significant variation of the pressure distribution with changes in $M_{\infty}$ and $\gamma$ over the range of conditions included in the calculations.

The pressure at the nose of the magnetosphere provided by the detailed calculations agrees, as it must, with the gasdynamic expression

$$
\mathrm{p}_{\mathrm{st}} / \rho_{\infty} \mathrm{v}_{\infty}^{2}=[(\gamma+1) / 2]^{(\gamma+1) /(\gamma-1)}\left[\gamma-(\gamma-1) / 2 \mathrm{M}_{\infty}\right]^{-1 /(\gamma-1)} / \gamma
$$

which simplifies for $\gamma=5 / 3$ to $\mathrm{p}_{\mathrm{st}} / \rho_{\infty} \mathrm{v}_{\infty}{ }^{2}=0.881\left[5 \mathrm{M}_{\infty}{ }^{2} /\left(5 \mathrm{M}_{\infty}{ }^{2}-1\right)\right]^{3 / 2}$. Since the free-stream Mach number $\mathrm{M}_{\infty}$ is much greater than unity in the present applications, comparison with the Newtonian expression for the pressure at the magnetosphere nose, $\mathrm{p}_{\mathrm{st}}=\mathrm{K}_{\infty} \mathrm{v}_{\infty}{ }^{2}$ as indicated in figure 12 , reveals that $K$ approaches 0.881 . The corresponding values for $\gamma=2$ and $\gamma=3 / 2$ are 0.844 and 0.904 . Since the distance $D$ to the nose of the magnetosphere is proportional to the inverse sixth root of $\mathrm{K}$, it follows immediately that the size as well as the shape of the magnetosphere is very insensitive to variations of the Mach number and ratio of specific heats of the solar wind gas.

One of the assumptions fundamental to the attainment of the numerical results presented in the preceding pages is that the Alfvén Mach number is sufficiently greater than unity that the equations of magnetohydrodynamics can be approximated satisfactorily by those of gasdynamics for the computation of the flow field and that the magnetic field can be calculated subsequently by application of the concept of the magnetic field lines moving with the fluid as outlined on figure 11. Comparison of the values for the Alfvén speed indicated on figure 10 with representative values for the velocity of the solar wind assures that this condition is easily met in the incident solar wind. It is more difficult to make a similar a priori comparison for the conditions behind the bow wave because both the flow velocity and the Alfvén velocity vary considerably from place to place. Once the velocity, density, and magnetic field intensity have been calculated for any point, however, it is a simple matter to evaluate the Alfvén Mach number and determine to what extent the required criterion is achieved. The results of such an examination are presented in figure 35 for two orientations of the interplanetary magnetic field for the case of $M_{\infty}=8$ and $\gamma=5 / 3$. The results are given in the form of a ratio of the local Alfvén Mach number $M_{A}$ to the free-stream Alfvén Mach number $M_{A_{\infty}}$ in order to take advantage of the simple proportionality between the two quantities at any point in the flow field. If $M_{A_{\infty}}=10$, say, the results show that $M_{A}$ is greater than unity everywhere except in a small 

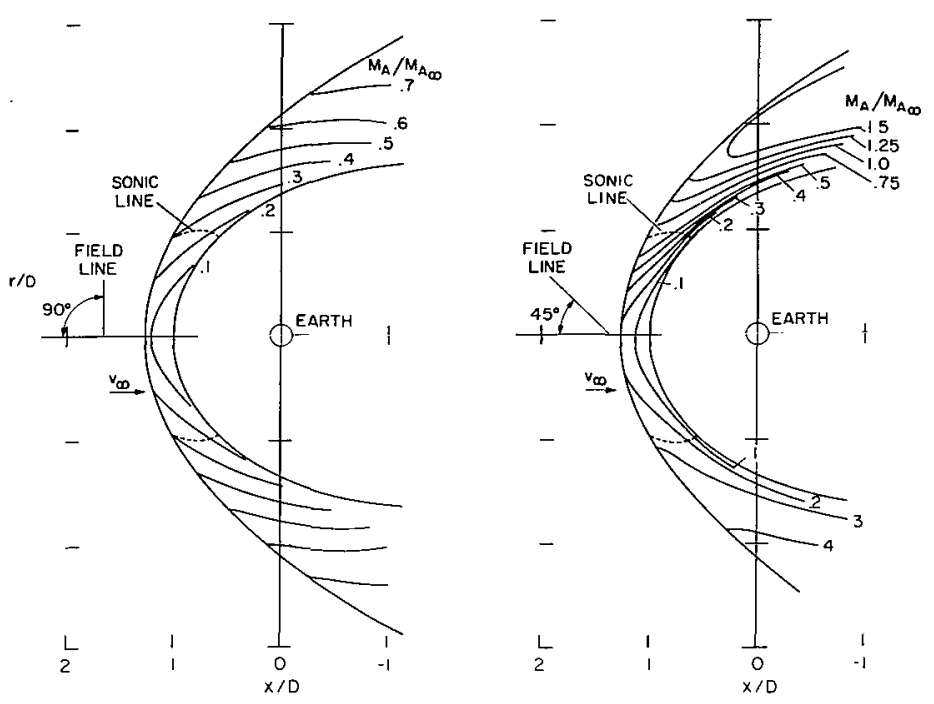

Figure 35. - Alfvén Mach number distribution; $M_{\infty}=8, \gamma=5 / 3$.

region near the magnetosphere nose. Although some localized departures from the calculated results might be anticipated in this region, it would appear that the larger scale features of the flow should be quite adequately represented by the gasdynamic and frozen-field calculations. The validity of the numerical results should be even more assured as $\mathrm{M}_{\mathrm{A}_{\infty}}$ increases beyond 10 and the critical region in which $M_{A}$ is less than unity decreases in size. As $\mathrm{M}_{\mathrm{A}_{\infty}}$ decreases below 10 , however, the critical region expands significantly, and increasing differences between the approximate numerical results and more exact theoretical or observational results should be anticipated. If $M_{A_{\infty}}$ should ever become as small as 2 or 3 , the critical region of small local Alfvén Mach number would become so extensive that substantial departures from the present results should be anticipated. Whether $\mathrm{M}_{\mathrm{A}_{\infty}}$ can actually attain such low values in the solar wind is not known, but the prospect of finding an extensive alteration in the basic flow pattern around the Earth should provide an incentive to examine observational data for this particular feature.

That the procedures employed in numerically solving the nonlinear equations of gasdynamics for the flow around the magnetosphere are indeed capable of yielding results that compare well with actual aerodynamic measurements is illustrated in figure 36. Shown in this figure is a shadowgraph photograph of a metal model of the magnetosphere in flight at a Mach number of 4.65 through an argon atmosphere having a ratio of specific heats $\gamma$ of $5 / 3$. The dark shadow of the bow wave is clearly visible somewhat upstream of the nose of the mode1. Superposed on the photograph is a dashed white line indicating the theoretical location of the bow wave computed for $M_{\infty}=4.65$ and $\gamma=5 / 3$. It may be seen that the agreement between the actual and computed locations of the bow wave is virtually perfect. Although the calculations were performed for the actual shape of the model, it should be noted that various experimental constraints required the shape of the model to deviate in certain particulars from the shape of the actual magnetosphere. Although these and other deviations should have no bearing on the validity of the present comparisons, they will be reviewed for the sake of completeness. 


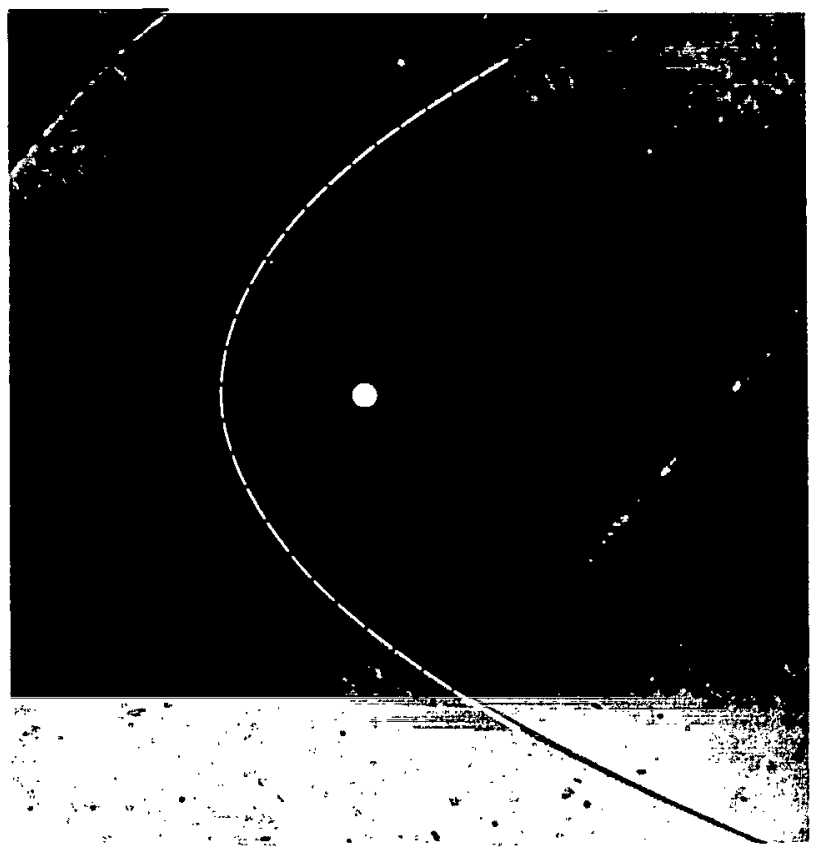

Figure 36. - Comparison of calculated and observed positions of bow wave of model magnetosphere in flight at Mach number 4.65 through argon; $\gamma=5 / 3$.
The principal constraints arise from the fact that the experiment was conducted by firing the model magnetosphere from a .50 caliber light-gas gun into the test chamber of the Supersonic Free Flight Wind Tunnel at Ames Research Center. In ordinary use of this facility, models are fired upstream through an elongated test section of an otherwise normal supersonic wind tunnel. The working fluid usually employed is air, but other gases can be used for a more $1 \mathrm{imited}$ range of test conditions that can be reached by shooting the models into stationary gas. Since the relative velocity between any given projectile and the gas is thus limited by the allowable muzzle velocity, the maximum Mach number that can be attained depends primarily on the speed of sound in the gas in the test section. By selecting argon as the gas, it is possible to obtain a value of $5 / 3$ for $\gamma$ and a low enough speed of

sound that Mach numbers as high as 4.5 or 5 can be achieved by firing the projectile into stationary gas. Although these conditions are not identical with those experienced by the actual magnetosphere past which flows ionized hydrogen at Mach numbers between, say, 5 and 12 or more, they are sufficiently close with respect to the two most important parameters of the analysis, namely, $\gamma$ and $M_{\infty}$, that the results should be useful and instructive.

Although the actual magnetosphere has an elongated shape because of its extended tail, and is not axisymmetric, the model had to be of modest length and axisymmetric to permit firing in the gun. Because of these requirements and in order to facilitate comparison with theoretical results, the model was contoured to the shape described by the equatorial trace of the magnetosphere boundary calculated by Spreiter and Hyett (1963) and Spreiter and Summers (1963) using a modified version of the Chapman-Ferraro theory in which the right-hand side of the boundary condition $\mathrm{H}^{2} / 8 \pi=\mathrm{pst}_{\mathrm{s}} \cos ^{2} \psi$ is replaced by $p_{s}+p_{s t} \cos ^{2} \psi$ in which $p_{s}$ is a constant. Results were given for many different values for the ratio $\mathrm{p}_{s} / \mathrm{p}_{\mathrm{s}}$; that selected for the experiment is $\mathrm{p}_{\mathrm{s}} / \mathrm{p}_{\mathrm{st}}=0.1$. The sunward portion of the resulting magnetosphere is very similar, although not identical, to that for $p_{s}=0$ used elsewhere in this paper. It follows from the basic rules of subsonic and supersonic flow that the location of the bow wave and details of the flow around the forward part of the magnetosphere are very nearly the same whether $\mathrm{p}_{\mathrm{s}} / \mathrm{p}_{\mathrm{st}}=0$ or 0.1 . The value for this parameter has a profound influence on the shape of the rear of the magnetosphere, however, and details of the flow may be expected to differ substantially in this region. The latter are not visible in figure 36, however, because this particular photograph was taken with a focussing 
shadowgraph system which has the property of portraying the shock wave with good resolution, but at the sacrifice of most other details of the flow. Most of the scattered isolated details that are, in fact, visible are without aerodynamic or geophysical meaning and are the result of damage to the viewing windows caused by impacting models and their fragments in the course of years of use of the facility.

While comparisons such as those shown in figures 34 and 36 and elsewhere tend to confirm the applicability and general consistency of the approximations introduced to permit the essential solution of the equations representing the fluid approach to the interaction of the solar wind and the geomagnetic field, an examination of conditions in the vicinity of the neutral points on the magnetosphere boundary described by Spreiter and Summers (1967) shows that certain alterations or refinements of the procedures are required. In a sense, the changes in conditions to be anticipated near the neutral points are all associated with the dual character of the expression $p=K \rho_{\infty} v_{\infty}{ }^{2} \cos ^{2} \psi$ for the pressure on the magnetosphere boundary, and the inadequacy, from the fluid point of view, of the results obtained therefrom when the angle $\psi$ between the direction of the undisturbed flow velocity and the outward normal to the magnetosphere boundary approaches 900 .

On the basis of numerous discussions and analyses of Chapman and Ferraro and others, the magnetic field lines comprising the magnetosphere boundary have long been considered to turn abruptly through $90^{\circ}$ at the neutral points and extend to the Earth as a single line. It follows from the equations $\operatorname{div} \underset{\sim}{\mathrm{H}}=0$ and $\underset{\sim}{\operatorname{cur}} \underset{\sim}{1} \underset{\sim}{\mathrm{H}}=0$ listed for the interior of the magnetosphere on figure $\tilde{1} 2$ that the magnetic field $\underset{\sim}{H}$ must vanish at the neutral points. As is frequently observed (see, for instance, Dungey, 1958), this result when combined with the boundary condition $\mathrm{H}^{2} / 8 \pi=\mathrm{p}_{\mathrm{st}} \cos ^{2} \psi$ implies that $\cos \psi=0$, or $\psi=90^{\circ}$. Since it is also required that $\cos \psi>0$ at all other points, this result leads to indentations in the magnetosphere boundary in the vicinity of each neutral point, as can be seen in the meridian plane traces of the boundary presented in figures 15 through 20 .

Completely apart from matters concerning the degree to which the condition $\cos \psi=0$ is satisfied, the shape of the magnetosphere boundary must actually differ significantly from that illustrated in the vicinity of each neutral point. Kendall (1962) discussed the situation within the framework of free particle flow, and observed that the shape of the boundary immediately downstream from the neutral points must be modified because of the additional pressure resulting from the second impact of particles that have ricocheted off more upstream elements of the surface. The situation is more complex when examined from the fluid point of view, but we may commence the discussion by noting that walters (1966) has pointed out that the presence of a concave curve or corner on the magnetosphere boundary in a region of supersonic flow would result in the formation of a shock wave attached to the magnetosphere boundary.

The shadowgraph in figure 37 illustrates such an attached shock wave in a flow with a free-stream Mach number of 5.03 and a ratio of specific heats of $\gamma=5 / 3$ about a simplified magnetosphere shape with a concave corner. This photograph was taken in an experiment conducted in the same way as described 


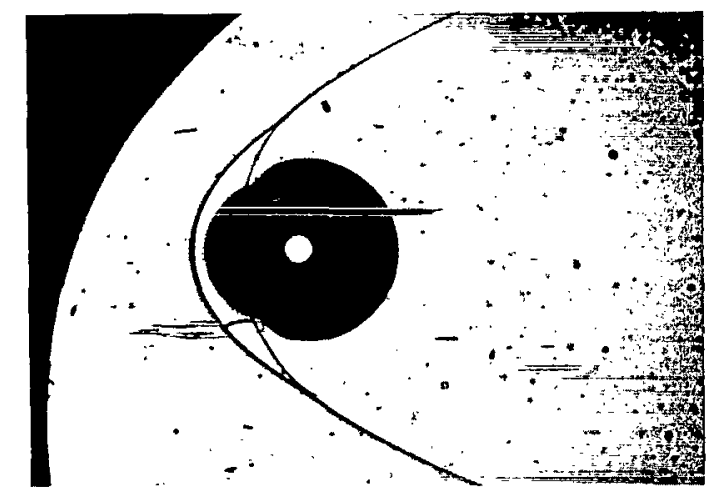

Figure 37.- Focussed shadowgraph of model of magnetosphere in flight at Mach number 5.03 through argon; $\gamma=5 / 3$. for figure 36 . The results differ because the mode1, while still axisymmetric, was contoured to correspond to the principal meridian, rather than the equatorial, trace of the magnetosphere boundary .

Although an attached shock wave is thus to be expected in supersonic flow around a solid obstacle with indentations like those of the theoretical magnetosphere shapes illustrated in figures 15 through 20 , it is not what is to be expected in high Mach number flow around the actual magnetosphere. The reason is that the magnetosphere

is not a solid obstacle of fixed geometry, but a surface that must adjust its shape in such a way that the balance between gas and magnetic pressure be maintained in accordance with the approximate relation $\left(\mathrm{H}^{2} / 8 \pi\right)$ mag $=p_{f l o w}$ given on figure 12 for a tangential discontinuity. Now the presence of an attached shock wave requires that there be a discontinuous change in the gas pressure on the magnetosphere boundary at the foot of the shock wave. Since

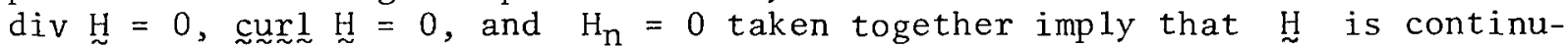
ous, there can, however, be no balancing discontinuity in the magnetic pressure $\mathrm{H}^{2} / 8 \pi$.

Additional difficulties occur if the attached shock wave is oblique to the magnetosphere boundary, as it appears to be in figure 37 , because the flow, and therefore the magnetosphere boundary as well, would be required to turn a sharp corner. A curl-free and divergence-free magnetic field would be either zero or infinite at the apex, depending on whether the corner were concave or convex toward the magnetosphere interior. Correspondingly, the magnetic pressure would be either zero or infinite at the corner, and a second condition would be imposed that would be impossible to achieve with a gas pressure. A further difficulty with the fluid extension of the conventional theory of the magnetosphere boundary is that the latter indicates that $\mathrm{H}=0$ at the neutral points. The boundary condition expressing the pressure balance would therefore require that the pressure $p$ vanish at these points, but this is also impossible in a gas. A step toward the resolution of this contradiction is suggested by considering a magnetosphere boundary model in which the Newtonian pressure formula $\mathrm{p}=\mathrm{K}_{\infty} \mathrm{v}_{\infty}{ }^{2} \cos ^{2} \psi$ is replaced by $\mathrm{p}=\mathrm{p}_{\mathrm{s}}+\mathrm{K} \rho_{\infty} \mathrm{v}_{\infty}{ }^{2} \cos ^{2} \psi$ where $\cos \psi \geq 0$ and by $\mathrm{p}=\mathrm{p}_{\mathrm{s}}$ where $\cos \psi \leq 0$. As described previously (Spreiter and Hyett, 1963; Spreiter and Summers, 1963; Slutz and Winkelman, 1964), the magnetosphere boundary defined by this model contains a sharp cusp directed toward the Earth at each neutral point for all values for $\mathrm{p}_{\mathrm{s}} / \mathrm{K}_{\rho_{\infty}} \mathrm{v}_{\infty}{ }^{2}=\mathrm{p}_{\mathrm{s}} / \mathrm{p}_{\mathrm{s} t}$ except zero. Although this result is consistent with the requirements of the free-particle approach, and has been proposed again more recently by Grad and Hu (1966) to be the appropriate configuration to consider for this region, it is not an admissible solution from the fluid point of view because the flow cannot follow the cusped contour without developing a shock wave. 
Spreiter and Summers (1967) have observed that the difficulties may be resolved if the boundary of the flowing solar plasma is considered to separate from the boundary of the geomagnetic field so as to form an enclosed region of finite extent filled with essentially stationary gas at constant pressure $p_{c}$, as illustrated in figure 38. Since the tangential velocity components are different on the two sides of the free streamline that divides the flowing

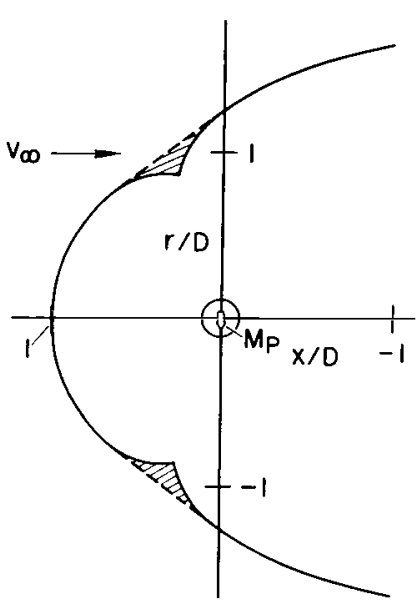

(a)

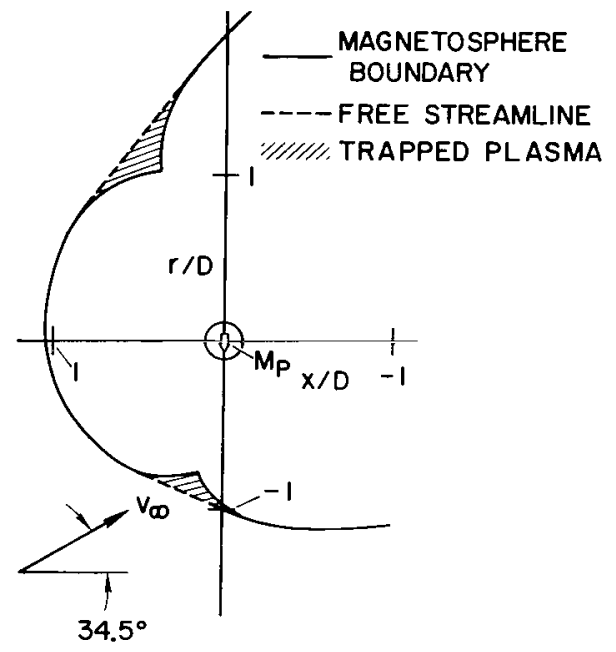

(b)

Figure 38.- Cusp-shaped regions of hot plasma in the vicinity of neutral points.

solar plasma from the stationary trapped plasma, and the normal velocity component is zero, conditions on the two sides of the free streamline are considered to be in accordance with those of a tangential hydromagnetic discontinuity outlined on figure 9. Arbitrary changes in density and tangential components of the velocity and magnetic field are allowed across such a surface, but the normal component of the magnetic field must be zero, and the sum of the gas pressure $p$ and the magnetic pressure $H^{2} / 8 \pi$ must be the same on both sides of the surface. Although the magnetic field is considered to be sufficiently strong to provide the necessary collective interaction between the individual particles of the rarefied plasma to enable it to act like a fluid, it is nevertheless considered to be sufficiently weak that the magnetic pressure is otherwise negligibly small compared with the gas pressure on both sides of the discontinuity. With previous theory retained as much as possible, it follows that $p_{c}=p_{s t} \cos ^{2} \psi_{f}$ where $\psi_{f}$ is the angle $\psi$ associated with the free streamline, and the magnetic field is such that $\mathrm{H}_{\mathrm{c}}{ }^{2} / 8 \pi=\mathrm{p}_{\mathrm{c}}=$ const and $\mathrm{H}_{\mathrm{n}}=0$ along the portion of the magnetosphere boundary adjacent to the trapped plasma. Over the remainder of the magnetosphere boundary, the conditions specified on figure 12 are to be applied in the usual manner.

Although the exact solution of this problem has not been determined, an approximate procedure has been described by Spreiter and Summers (1967) that leads to the results for the shape of the principal meridian trace of the magnetosphere boundary illustrated in figure 38. As can be seen, the stream1ine bounding the flowing plasma coincides with the magnetosphere boundary from the stagnation point at the nose to a separation point, situated somewhat upstream of the neutral point. The flow then detaches from the boundary of 
the geomagnetic field and follows a free streamline of constant pressure until it reattaches to the magnetosphere boundary at a point somewhat downstream of the neutral point. The relation $p_{c}=p_{s t} \cos ^{2} \psi=$ const indicates that the free streamline must be a straight line in the principal meridian plane. It must also be tangential to the magnetosphere boundary at both the detachment and reattachment points since otherwise unwanted discontinuities or singularities in the pressure would occur that could not be balanced satisfactorily by the magnetic pressure within the magnetosphere. Although the approximate results shown in figure 38 do not display the feature adequately, the portion of the magnetosphere boundary adjacent to the stationary plasma must be sufficiently sharply cusped that $|\underset{\sim}{H}|$ is constant and different from zero at all points.

Since the gas trapped in the cusped regions must have come originally from the solar wind, its temperature $T_{C}$ can be estimated to be the same as the stagnation temperature of the solar wind. The latter is given in terms of the temperature $T_{\infty}$ and Mach number $M_{\infty}$ of the incident solar wind by the expression $\mathrm{T}_{\mathrm{C}}=\mathrm{T}_{\infty}^{\infty}\left[1+(\gamma-1) \mathrm{M}_{\infty}^{2} / 2\right]$ obtained from the more general expression given on figure 23 by setting $v / v_{\infty}$ to zero. The temperature of the trapped gas is thus independent of the relative orientation of the dipole axis and the free-stream direction of the solar wind, and is equal, for example, to $22.3 \mathrm{~T}_{\infty}$ when $M_{\infty}=8$ and $\gamma=5 / 3$. The number density $n_{c}$ of the trapped protons can be estimated by combining the above expression for $\mathrm{T}_{\mathrm{c}}$ with the relation $p_{c}=2 n_{c} k T_{c}=\rho_{\infty} v_{\infty}^{2} \cos ^{2} \psi_{f}$, in the derivation of which the coefficient $K$ has been equated to unity for simplicity. The resulting expression for the ratio $\mathrm{n}_{\mathrm{c}} / \mathrm{n}_{\infty}$ of the number density of trapped protons to that of the incident solar wind is $n_{c} / n_{\infty}=\left(2 \gamma M_{\infty}{ }^{2} \cos ^{2} \psi_{f}\right) /\left[2+(\gamma-1) M_{\infty}^{2}\right]$. For flow with $M_{\infty}=8$ and $\gamma=5 / 3$, Spreiter and Summers (1967) find that $\psi_{f}=53^{\circ}$ and $\mathrm{n}_{\mathrm{c}} / \mathrm{n}_{\infty}=1.73$ when the dipole axis is normal to the direction of the solar wind. When the direction of the solar wind is inclined $34.5^{\circ}$, the corresponding values are $33^{\circ}$ and 3.34 in the summer hemisphere and $73^{\circ}$ and 0.39 in the winter hemisphere.

Each trapping region may be recognized to have essentially the same shape as the axisymmetric cusped geometry configurations considered in studies of plasma containment for production of thermonuclear energy. The major advantage of the cusped configuration is its stability. One of its chief disadvantages is the large rate of loss of particles. Since a large loss rate could have important geophysical consequences, both as a mechanism for injecting particles into the magnetosphere in general, and as a source of particles that spiral down along the field lines from the neutral points to localized regions in the Earth's atmosphere, it is of interest to inquire into the loss rate that might be expected. This has been done by Spreiter and Summers (1967) using a basic expression given by Grad (1963) for the proton loss rate $u$ at an axisymmetric cusp for an isotropic distribution of particles of a single speed $V_{+c}$ and density $n_{c}$. It is that $u=45\left(m_{-} / m_{+}\right)^{1 / 2} n_{c} V_{+c} \pi \lambda^{2} / 4$ in which $\mathrm{m}_{-}=9.11 \times 10^{-28} \mathrm{gm}$ and $\mathrm{m}_{+}=1.67 \times 10^{-24} \mathrm{gm}$ represent the mass of an electron and a proton, and $\lambda=\mathrm{m}_{+} \mathrm{V}_{+\mathrm{c}} \mathrm{c} / \mathrm{eH} \mathrm{c}$, in which $\mathrm{e}=4.8 \times 10^{-10}$ esu is the charge on an electron and $c=3 \times 1010 \mathrm{~cm} / \mathrm{sec}$ is the speed of 1 ight, represents the gyro radius of a proton moving with speed $v_{+c}$ in a magnetic field of intensity $\mathrm{H}_{\mathrm{C}}$. Grossmann (1966) has recently reexamined Grad's problem in 
greater detail and arrived at an expression for $u$ that differs somewhat in numerical coefficient but is otherwise the same as that presented above. The corresponding Maxwel1 averaged loss rate $\langle u\rangle$ follows directly upon replacement of $\mathrm{n}_{\mathrm{c}} \mathrm{V} \mathrm{F}_{\mathrm{c}}$ by $3 \mathrm{nkT} / \mathrm{m}_{+}$in the expression for $u$. Insertion of the appropriate relations for conditions in the trapped plasma leads to the expression $\langle u\rangle=2.8 \times 10^{14}[(\gamma-1) / \gamma]^{1 / 2} v_{\infty}$. For representative values of $v_{\infty}=5 \times 10^{7} \mathrm{~cm} / \mathrm{sec}$ and $\gamma=5 / 3^{\infty}$, this expression indicates a loss rate of $8.9 \times 10^{21}$ protons $/ \mathrm{sec}$ from each cusp. This rate is, moreover, independent of the number density of the solar wind and the relative orientation of the dipole axis and the direction of the solar wind. The area $A=45 \pi \lambda^{2}$ over which the particle flux is distributed near the tip of the cusp is found to be given by $A=2.8 \times 10^{16}(\gamma-1) /\left(\gamma n_{\infty} \cos ^{2} \psi_{f}\right)$ for free-stream Mach numbers much greater than unity. The flux density of protons per unit area $<U>/ A$ out of the vicinity of the tip of the cusp is thus proportional to the solar wind flux $\mathrm{n}_{\infty} \mathrm{v}_{\infty}$ and exhibits substantial seasonal variations in accordance with the values for $\cos ^{2} \psi_{f}$. With $\langle u\rangle / A$ normalized to unity when the dipole axis is perpendicular to the direction of the solar wind, this result when combined with the values for $\psi_{f}$ given above shows that the flux density through the summer cusp is approximately 2 and that through the winter cusp is approximately $1 / 4.5$ when the direction of the solar wind is inclined 34.50 to the plane normal to the dipole axis. Since, in addition, the density of the solar wind may vary by a factor of 10 or more and the velocity by a factor of 3 or more in time intervals as short as a few minutes, changes in the values of $<u>/ A$ by a factor of 30 or more are to be anticipated in correspondingly short time intervals. The total flux of protons from each cusp would vary much less, however, because it depends only on $v_{\infty}$.

The possible existence of particle fluxes into the magnetosphere near the neutral points appears to be supported by laboratory experiments designed to simulate the interaction of the solar wind and the geomagnetic field. Results obtained by independent groups in Canada (Osborne, Bachynski, and Gore, 1964), Japan (Kawashima and Fukushima, 1964), and USA (C1adis, Miller, and Baskett, 1964; Waniek and Kasai, 1966) have all displayed injection of plasma into the magnetosphere from cusp-shaped regions extending toward the Earth from the vicinity of the theoretical neutral points.

Ground-based observational evidence that such phenomena may actually occur is provided by magnetometer records from the polar regions. A considerable body of data gathered during the IGY (July 1957-December 1958) and the Second Polar Year (August 1932-August 1933) has been assembled and analyzed by Fukushima (1962) in various ways according to the time of day and year, and the degree of disturbance of the worldwide geomagnetic field. The results show that the polar regions are constantly disturbed, even at periods when the planetary geomagnetic field is very quiet. Two of Fukushima's many summary plots are reproduced in figure 39. They show the latitudinal distribution of the average geomagnetic disturbance near the noon-midnight geomagnetic meridians for the case of $\mathrm{Kp}=0$, that is, for the very quietest times as indicated by the planetary geomagnetic index, at two selected sets of times extending over the entire period of the IGY. The results show that the magnitude of the polar-cap disturbance is of the order of a few or several tens of gammas, that both polar caps are equally disturbed when the geomagnetic dipole axis is p erpendicular to the Sun-Earth line $(\lambda=0)$, and that the disturbances 

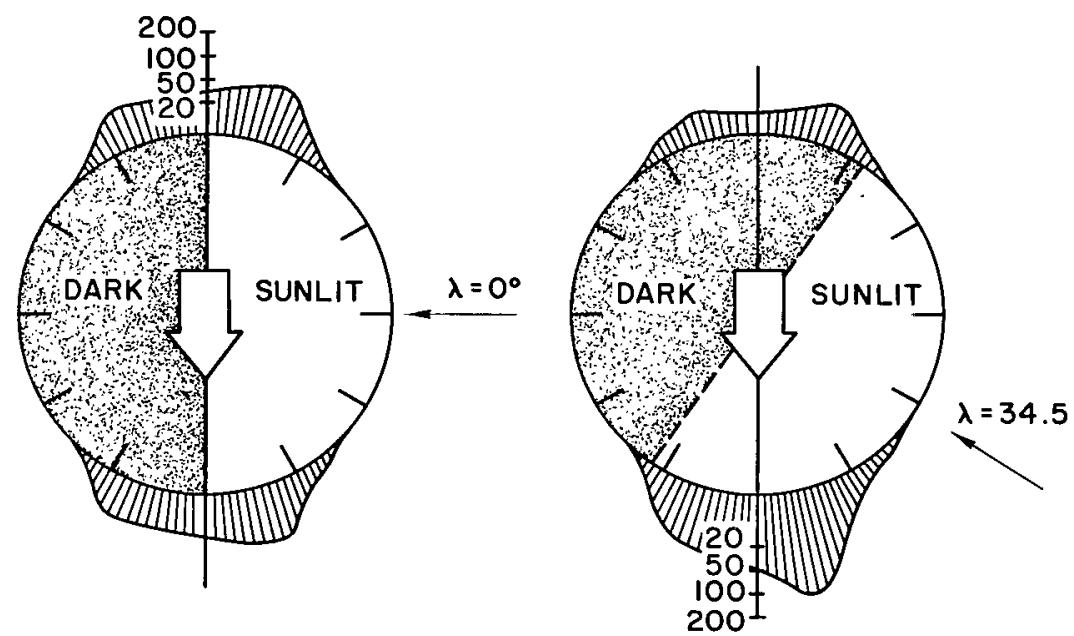

Figure 39.- Polar magnetic disturbance on very quiet days $(\mathrm{Kp}=0 \mathrm{o})$ during IGY (Fukushima, 1962 ).

are a maximum at summer noon and a minimum at winter midnight. These results, as well as the location of the maximum disturbances, appear to be consistent with what might be anticipated on the basis of direct invasion of charged particles or propagation of hydromagnetic waves into the polar regions along the fields from the neutral points on the magnetosphere boundary.

The results described above are also consistent with several inferences drawn by Lebeau (1965) from analysis of diurnal magnetic activity at a number of stations at very high latitudes in the arctic and antarctic. An important conclusion of his studies is that stations within a large portion of the polar cap display a diurnal variation of magnetic activity that peaks strongly at a time midway between local noon and magnetic noon. This is explained in terms of a model in which the intensity of the magnetic activity is determined by the combined action of two separate factors, an "excitation factor" which tends to create a maximum at magnetic noon and a "modulation factor" which is controlled by local time. The latter is considered to result from solar

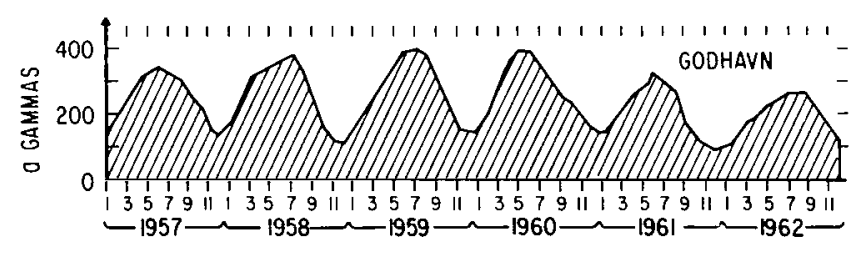
influences on the conductivity of the E-region of the ionosphere. The former is presumed to be the result of particle precipitation, and displays many properties that are consistent, perhaps even quantitatively, with the

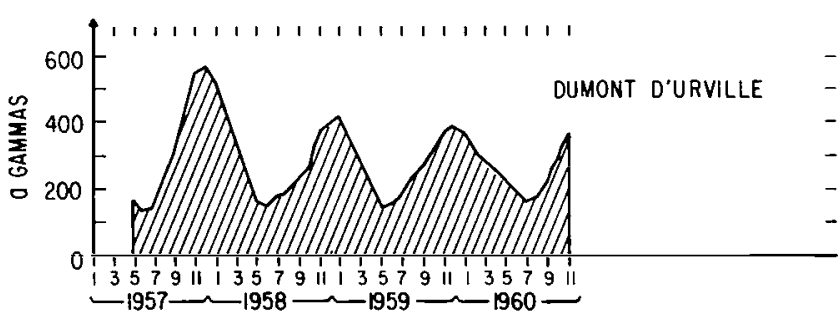
theoretical results described above. From figure 40 reproduced from Lebeau (1965), it may be seen, for instance, - that the seasonal variation of the

- amplitude of magnetic activity at

- Godhavn in the arctic is almost perfectly out of phase with that at Dumont d'Urville in the antarctic

Figure 40.- Amplitude of magnetic activity for an arctic station, Godhavn, and an antarctic station, Dumont d'Urville (Lebeau, 1965). with the maximum activity occurring at each station during the months of local summer. Values between about 
3 to 5 for the enhancement of the amplitude of the activity observed in midsummer months over that at the same station in the midwinter months are compatible with the seasonal variations of the proton flux density calculated by Spreiter and Summers (1967). Although the latter have not been evaluated explicitly for Godhavn or Dumont d'Urville, the seasonal variation should be somewhat smaller than the value of 9 quoted above because geometrical factors tend to prevent observation at a single station of the maximum value of the excitation factor in the entire summer polar cap and the minimum value in the entire winter polar cap. The amplitude of the magnetic variation at Godhavn may also be seen to vary recognizably with the solar cycle, but with a range that is substantially less than the seasonal variation. As an example, mean values for July 1962 were only about 70 percent of those for July 1959 . Such a change would correspond, assuming a linear relation between the amplitude of the magnetic activity and the injection rate, to a value for the mean flux density $\rho_{\infty} v_{\infty}$ of the solar wind about 70 percent as large in 1962 as in 1959. Although direct measurements of the density and velocity of the solar wind were not made in 1959, this result is reasonable in terms of known variations in the solar wind and its interaction with the Earth's magnetic field.

\section{COMPARISON WITH OBSERVATIONS IN SPACE}

The final test of any theory of the interaction between the solar wind and the Earth must be based on comparisons with observations actually made in space. Such comparisons have already been made by several different groups on several levels of detail. These range from simple comparisons of gross features such as the existence and location of the magnetosphere boundary and the bow shock wave to more detailed comparisons of features such as the velocity, density, and temperature of the flowing plasma and the intensity and direction of the magnetic field. It is inevitable that even finer details such as perhaps the precise form of the velocity distribution of the particles as well as temporal fluctuations and changes of the bulk quantities will be compared as further development of the theory, instrumentation, and data processing techniques continues.

Many of the earlier comparisons involving the bow wave made use of the theoretical results of Spreiter and Jones (1963) shown here in figure 41. Included in this figure are the equatorial trace of the magnetosphere boundary for $\lambda=0$ given previously in figures 15 and 16 together with the corresponding shape of the bow wave for axisymmetric flow of a perfect gas approaching the Eart" at a Mach number of 8.71. Only the position of the bow wave for $\gamma=2 \mathrm{w}$. jiven originally, but the corresponding results for $\gamma=5 / 3$ were made available shortly later in IG Bulletin 84 (1964). Since it was an important part of the original purpose in making these calculations to determine the expected location in space of the bow wave and the magnetosphere boundary, the results were given in dimensional, rather than in nondimensional, form. Values for the number density $n_{\infty}$ of the protons and their bulk velocity $v_{\infty}$ in the incident solar wind were selected as indicated on the figure, although the results would be identical for any other combination of values that leads to the same product $n_{\infty} v_{\infty}^{2}$. They could also be changed simply to correspond to any other value for this product by altering all linear 


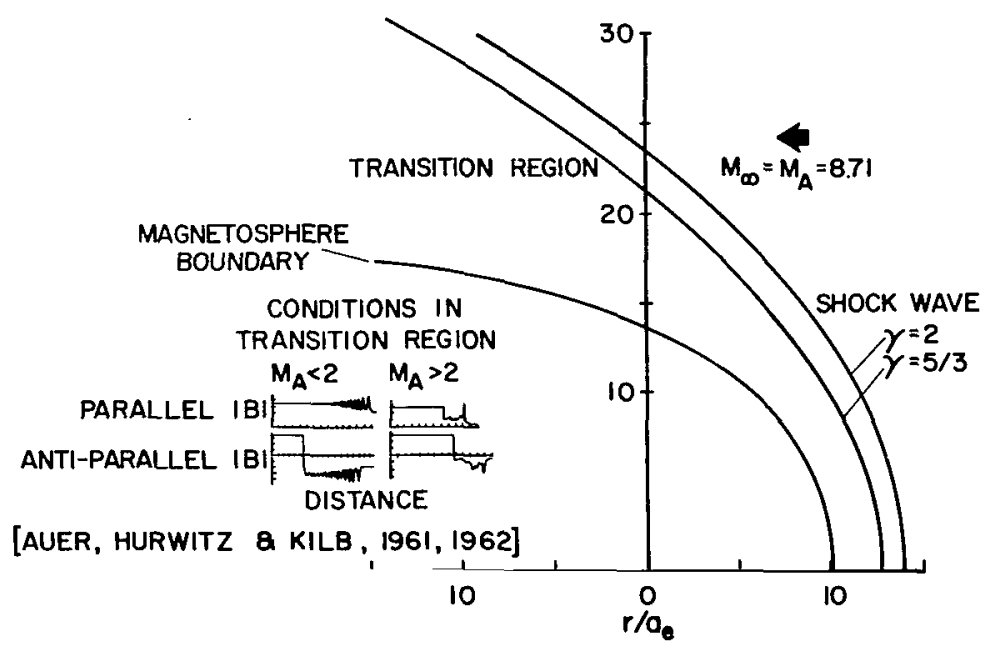

Figure 41.- Calculated shape of shock wave and suggested nature of conditions in the transition region; $\mathrm{n}_{\infty}=2.5$ protons $/ \mathrm{cm}^{3}, v_{\infty}=600 \mathrm{~km} / \mathrm{sec}, \mathrm{B}_{i}=5$ ganmas .

dimensions in proportion to the ratio of the inverse sixth root of $\mathrm{n}_{\infty} v_{\infty}{ }^{2}$. The value of 8.71 for the Mach number was selected so as to be equal to the Alfvén Mach number corresponding to the values for the density, velocity, and magnetic field indicated on the figure. As is evident from the discussion associated with figure 11 , there is, however, no need to equate the Mach number to the Alfvén Mach number. A large value for the latter merely insures that the equations of magnetohydrodynamics can be approximated by the simpler, though still complicated, equations of gasdynamics. A value of 8.71 for the Mach number is, nevertheless, both reasonable and sufficiently great that the location of the bow wave does not depend critically on the Mach number.

Also retained on figure 41 are small insert plots suggested to be indicative of possible appearances of the magnetic field in the region between the bow wave and the magnetosphere. These were reproduced from results calculated by Auer, Hurwitz, and Kilb $(1961,1962)$ for the detailed magnetic structure of the transition region between a steady magnetic field and an adjacent plasma containing a smaller magnetic field. Results of similar calculations for a much wider range of conditions have been given subsequently by Rossow (1965, 1967) and Jones and Rossow (1965). Although all of these calculations are for one-dimensional unsteady flow and the geophysical application is considered here as a three-dimensional steady flow problem, the conditions at any given instant after the one-dimensional interaction zone has become of sufficient width should be approximately equivalent to those actually encountered in space between the magnetosphere boundary and the bow wave. These results illustrate that a value of 2 for the Alfvén Mach number based on the velocity component normal to the shock wave is critical, and that conditions associated with values on either side of 2 are strikingly different. If $M_{A}<2$, as occurs well back along the shock wave in general, and possibly near the nose if the interplanetary magnetic field is sufficiently strong, the magnetic fields calculated by Auer, Hurwitz, and Kilb $(1961,1962)$ are oscillatory and orderly. A pair of examples for parallel and antiparallel geomagnetic and interplanetary fields is shown in the small insert figures for $M_{A}=1.54$. 
Although the magnetic field variations in the transition region are similar in form for the two cases, the results are distinguishable because of the change in direction of the field at the magnetosphere interface. Particularly significant is the fact that the absolute value of the magnetic field undergoes no significant change at the boundary of the magnetosphere. If $M_{A}>2$, the magnetic field profile at any instant is very irregular, as is shown in the remaining pair of small inserts in figure 41. They are for an example in which $M_{A}=5.8$ and the interplanetary field is either parallel or antiparallel to the geomagnetic field. Of particular significance is the fact that the irregular field calculated for the transition region retains the same direction as the interplanetary field and has an average value that may be much less than the intensity of the field at the boundary of the magnetosphere.

Figure 42 shows some observational results obtained by Cahill and Amazeen (1963) with Explorer 12 that bear close resemblance to the results of Auer, Hurwitz, and Kilb (1962) for $M_{A}>2$. The continuous line labeled

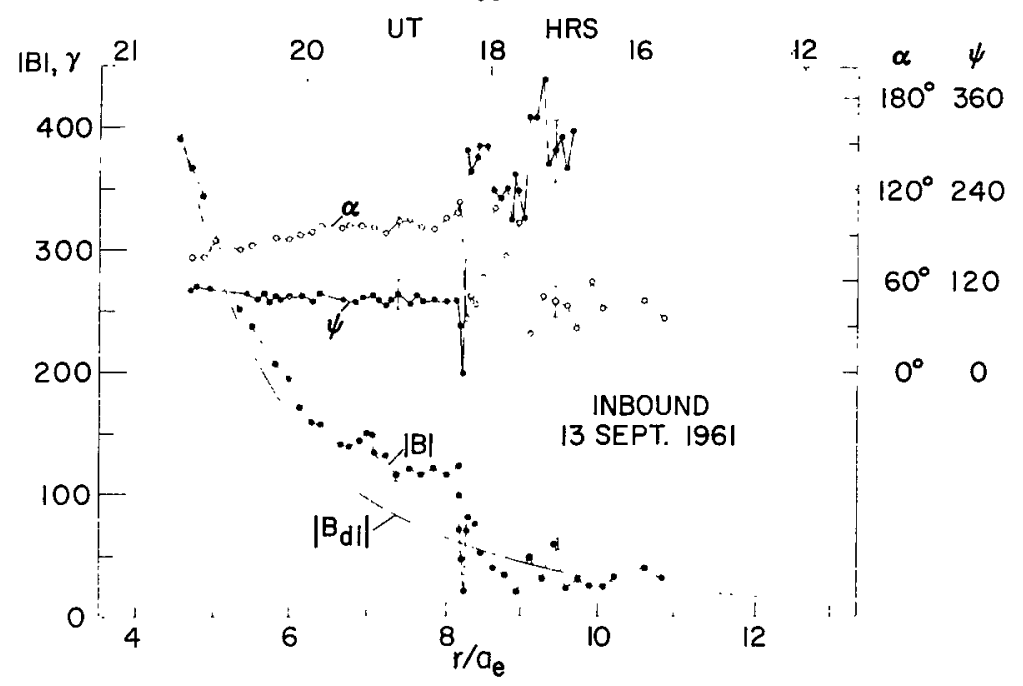

Figure 42.- Explorer XII data resembling calculated results for antiparallel fields with $M_{A}>2$
(Cahill and Amazeen, 1963).

$\left|B_{d i}\right|$ represents the values for the intensity of the geomagnetic field calculated by harmonic expansion of the surface values according to the analysis of Finch and Leaton (1957). Because of the relatively large values for $r / a_{e}$, the calculated intensities are very nearly those provided by the geomagnetic dipole term alone, since the other terms are not only smaller at the Earth's surface but diminish more rapidly with increasing altitude. The data points and broken curves represent the measured values for the intensity $|B|$ and the direction of the actual magnetic field. The angle $\alpha$ lies between the direction of the magnetic field and the spin axis of the spacecraft. The angle $\psi$ lies between the plane containing the magnetic field and the spin axis and the plane containing the Sun direction and the spin axis. Each data point shown represents the average of 20 individual measurements made at the rate of approximately 3 per second, and only a small fraction of even these values is included on the plot. Moreover, each component of the individual measurements contains an uncertainty of $\pm 12 \gamma$ resulting from digitization of the data. The boundary of the magnetosphere, plainly visible at about 8.2 Earth radii, was traversed by Explorer 12 at a geomagnetic latitude of 
$+13.0^{\circ}$ at a 1ocal time of 1200 on September 13, 1961. The geomagnetic field observed at this time on the Earth's surface was moderately disturbed with several observatories reporting that a moderately severe magnetic storm commenced suddenly a few hours earlier at 1554 UT (Lincoln, 1962).

A noteworthy feature of the results shown in figure 42 is that the boundary of the magnetosphere is defined as much or more by the abrupt change in direction (note that $\psi$ changes by approximately $180^{\circ}$ ) as by the change in the intensity of the field. Just inside the magnetosphere, the magnetic field is relatively constant and of about twice the intensity of the extrapolated surface field, as assumed in the approximate calculation of the shape of the magnetosphere boundary outlined on figure 13. Beyond the boundary, the field is irregular, much as calculated by Auer, Hurwitz, and Kilb (1962) for $\mathrm{M}_{\mathrm{A}}>2$. No evidence of passage through the bow wave is apparent in the data presented in figure 42, but it is quite likely that this is because the bow wave is farther from the Earth than the greatest distance for which data are indicated.

The calculated results for $M_{A}<2$ also have a counterpart in the data presented by Cahil1 and Amazeen (1963) and illustrated here in figure 43 . On

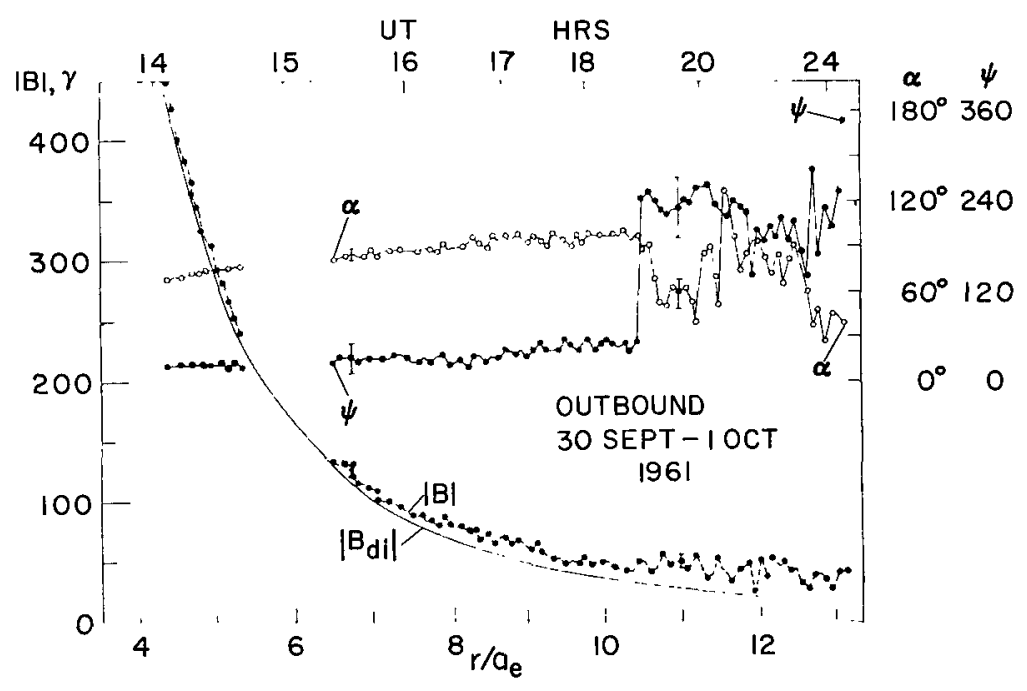
Figure 43.- Explorer XII data resembling calculated results for antiparallel fields with $M_{A}<2$
(Cahill and Amazeen, 1963).

this occasion Explorer 12 crossed the magnetosphere boundary at a geomagnetic latitude of $-18.2^{\circ}$ at a local time of 0822 . As in figure 42 , there is again a sudden marked change in the direction of the magnetic field at the magnetosphere boundary at 10.6 Earth radii. There is, however, no significant change in intensity. Farther from the Earth, the magnetic field appears to develop an oscillatory character that resembles, at least in broad outline, the calculated results of Auer, Hurwitz, and $\mathrm{Kilb}(1961,1962)$ for $\mathrm{M}_{\mathrm{A}}<2$. In both figures 42 and 43 , it may be observed that data from Explorer 12 more nearly resemble the calculated results for antiparallel rather than parallel fields. 
Detailed analysis of the magnetometer data obtained over the entire useful 1ife of Explorer 12, which extended from August 16 to December 6, 1961, has revealed many other important features of the outer magnetosphere and the surrounding flow. Mead and Cahill (1967) have examined the data covering the region from $3-1 / 2$ to 13 Earth radii for seven passes during geomagnetically quiet times, and made comparisons both with the Jensen and Cain (1962) harmonic representation of the contributions of internal sources and the Mead (1964) bounded magnetosphere field model. Near the noon meridian and from 6 or 7 Earth radii to the boundary of the magnetosphere, the magnitude and direction of the observed field are shown to agree well with the results calculated using the latter model. Near the dawn meridian, a significant twisting of the field lines out of the dipole magnetic meridian plane is observed, corresponding to the tendency of the field to point in the antisolar direction in the southern hemisphere. On one pass north of the equator, the twisting is observed to be in the opposite sense from that expected, however. This result is interpreted as indicating that there may possibly exist large north-south magnetosphere asymmetries even when the solar wind is directed nearly perpendicular to the dipole axis.

More than 70 distinct magnetosphere boundary crossings by Explorer 12 have been analyzed extensively by Cahill and Patel (1967). The location of the boundary was compared with the theoretical position determined by Mead (1964), displayed here in figure 19, normalized to a geocentric distance to the magnetosphere nose of 10.7 Earth radii. The measurements are generally consistent with the theoretical predictions, but the day-to-day boundary fluctuations and the lack of an effective plasma probe on Explorer 12 preclude making a precise evaluation of the theory. The thickness of the boundary was reported to be usually between about 20 and $300 \mathrm{~km}$. Such distances are sufficiently small to justify the theoretical idealization of the boundary as a discontinuity surface for most purposes concerned with the external aerodynamics of the magnetosphere. On a few occasions, however, a sharply defined boundary is lacking, and thicknesses as great as several thousand kilometers are indicated if the boundary thickness is considered to be the entire distance over which slow changes from magnetosphere to transition region values occur.

Evidence of boundary motion was provided by the appearance of multiple boundary crossings, much like those observed earlier in the flight of Explorer 10, in 22 of the 44 passages examined in detail. Motion of the boundary by more than $1000 \mathrm{~km}$ was inferred from measurements on 8 passes, but smaller motions ( 100 to $1000 \mathrm{~km}$ ) appeared more frequently. Cahil1 and Patel (1967) have suggested that the boundary motion could, on occasion, be periodic. Evidence in support of the view that even the largest amplitude variations in the position of the magnetosphere boundary detected by Explorer 12 may be periodic is given by Freeman, Kavanagh, and Cahill (1967). The data are principally that obtained on the outward pass on August 12, 1961, when the satel1ite was in the fortuitous position to observe two complete cycles of a major boundary motion, together with more fragmentary results obtained on several other occasions. These data, limited though they may be, tend to suggest a periodicity of the order of $1 / 2$ hour. Whether such a presumed periodicity represents the natural or resonant period of the magnetosphere, or the response to a driving periodicity in the solar wind remains uncertain. Quite independently of the 
origin of these oscillations, Freeman, Kavanagh, and Cahill (1967) go on to suggest that they may be responsible for the 40 to 50 minute fluctuations found in the equatorial surface magnetic field by Pai and Sarabhai (1964).

On the other hand, absence of apparent boundary motion in half of the passages examined indicates that gross boundary instability is not a characteristic of the forward part of the magnetosphere boundary. These results do not preclude such instabilities from occurring along the boundary of the magnetosphere tail, however.

Cahill and Patel (1967) concluded from their analysis of the data that the boundary observations are generally consistent with the closed magnetosphere model of Chapman and Ferraro described in this paper, although the boundary may, on occasion, be open in the manner suggested by Dungey (1963). Additional support for these conclusions are provided in a related study of the magnitude of the magnetic field component normal to the magnetosphere boundary conducted by Sonnerup and Cahill (1967). This field component was found, with a few notable exceptions, to be very small compared with the tangential component. In particular, they concluded there is little overall agreement with the open magnetosphere prediction of Levy, Petschek, and Siscoe (1964) that the magnitude of the normal component should be at least 10 percent and more likely 20 percent of the total field intensity. This result lends substantial observational support to the use of a hydromagnetic tangential discontinuity surface, at which there is no normal component to the magnetic field, to represent the steady-state magnetosphere boundary. Data consistent with a rotational discontinuity surface such as has been associated with the open magnetosphere model were observed on two occasions, both of which occurred while a geomagnetic storm was in progress. Under such circumstances, the magnetosphere might be expected to be in a transient state of contraction or expansion, and the usual statements related to the steady-state magnetosphere boundary would not necessarily be expected to apply.

Because apogee of Explorer 12 was only about 13 Earth radii, the bow wave was traversed on only three orbits at times when the magnetosphere was in a highly compressed state. Kaufmann (1967) has examined the data on each of these occasions. The location of the bow wave relative to the magnetosphere and the change in the magnitude of the magnetic field across the shock are concluded to be consistent with the results of fluid calculations such as those presented here in figures 22 through 29. The appearance of fluctuations in the magnetic field as the bow wave is crossed when traveling in the direction toward the magnetosphere, and conversely, much as indicated by the small insert plots in figure 41, are interpreted as indicating the bow wave to be the source of disorder in the flow. The thickness of the bow wave was always thin compared with the overall dimensions of the magnetosphere, although difficult to specify with precision in an unambiguous manner because of the complexity of its structure. The data indicate the time interval between the instant the magnetic field is first observed to deviate from its interplanetary level and the instant when the quiescent field level first reaches the shocked value to vary between 30 seconds and the minimum resolving time of the measurements (less than 2 seconds). The proper conversion of time intervals into distances is not known with certainty, but the latter may be estimated by 
considering that the satellite is moving relative to the Earth at about $1 \mathrm{~km} / \mathrm{sec}$, and the shock is thought to be moving past the satellite with an average speed of the order of $10 \mathrm{~km} / \mathrm{sec}$. Most of the data are judged to be consistent with the interpretation that this region consists of a shock much thinner than just indicated, plus several other waves. In addition, one or more large oscillations having a period of the order of several tens of seconds are sometimes observed near the shock wave. A number of possible explanations for these oscillations are discussed, but it is concluded that no decision can be reached as to which is correct.

While the data from Explorer 12 together with the more fragmentary data from even earlier spacecraft served to suggest the possible existence of a bow wave upstream of the magnetosphere, it was the data from the magnetometers and plasma probes of Explorer 18, also known as IMP-1, that provided the definitive evidence. This spacecraft was launched from Cape Kennedy at 0230 UT on November 27, 1963, with an initial apogee of 31.02 Earth radii geocentric, perigee altitude of $192 \mathrm{~km}$, and an orbital period of 3.934 days. The initial Sun-Earth-apogee angle was approximately $27^{\circ}$ toward the dawn side of the Earth. The extremely eccentric orbit combined with the more than six months useful life of the spacecraft permitted mapping of the magnetosphere boundary and bow wave from near the subsolar point to far into the nighttime region of the Earth.

Clearly evident in much of the data are easily distinguishable differences between conditions in the magnetosphere, in the incident solar wind beyond the bow wave, and in the intervening transition region. As a result it became the practice of several of the experimenters to present summary plots showing the portion of the orbits in which the data displayed transition region characteristics together with the theoretical curves for the magnetosphere boundary and the bow wave reproduced here in figure 41 . In the case of the magnetometer data, this region is recognizable by the presence of fluctuations more substantial than in either the incident solar wind or in the magnetosphere, much

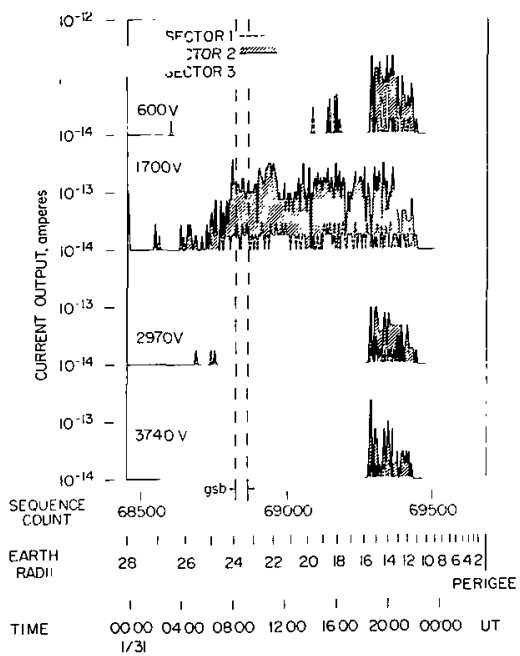

Figure 44. - IMP-1 plasma probe output - orbit 17, January 31-February 1, 1964 (Wolfe, Silva, and Myers, 1966). as indicated by the results shown in figures 41 through 43 . The differences are even more obvious in the data from the plasma probes. Figure 44 shows a sample of the latter, as presented by Wolfe, Silva, and Myers (1966), for the current output of their plasma probe during the inbound half of orbit 17 . Only the data from the four lowest voltage or energy steps of the probe are plotted, since current was seldom observed in the energy windows above 3740 volts ( $846 \mathrm{~km} / \mathrm{sec}$ for protons). This plot shows that no plasma flux was detected on this particular orbit when the spacecraft was nearer than about 11.5 Earth radii. This is because the spacecraft was within the magnetosphere, and hence shielded from the flowing 
solar plasma. When it was beyond about 16 Earth radii, essentially all the plasma flux was observed by only one of the energy channels of the probe. This is because the spacecraft was in the solar wind, beyond the disturbing influence of the Earth, where the random thermal velocities of the particles were sufficiently small compared with the directed bulk velocity that the velocity distribution or energy spectrum of the particles was too narrow to bridge the gap between the energy windows of the plasma probe. The lack of current output in al1 energy channels when the spacecraft was near apogee is interpreted on the basis of related data for other times and of design features of the probe to be due not to a temporary absence of the solar wind, but to a shift in the bulk velocity to a value such that the sensible part of the particle energy spectrum fell between the observing windows of the probe. Ground-based magnetometer data support this interpretation as well because they indicate that a gradual commencement geomagnetic storm occurred at approximately 0830 UT on January 31, 1964, when the spacecraft was at about 24 Earth radii. Presumably, then, the energy spectrum of the solar wind prior to this time would be such as to slip undetected between the 1700-volt and 600-volt channels of the instrument.

Although the magnetosphere boundary and bow wave were not always so clearly defined in the data as in the case of orbit 17, it was usually possible to distinguish the data from the various regions. Figure 45 is a summary plot THEORY

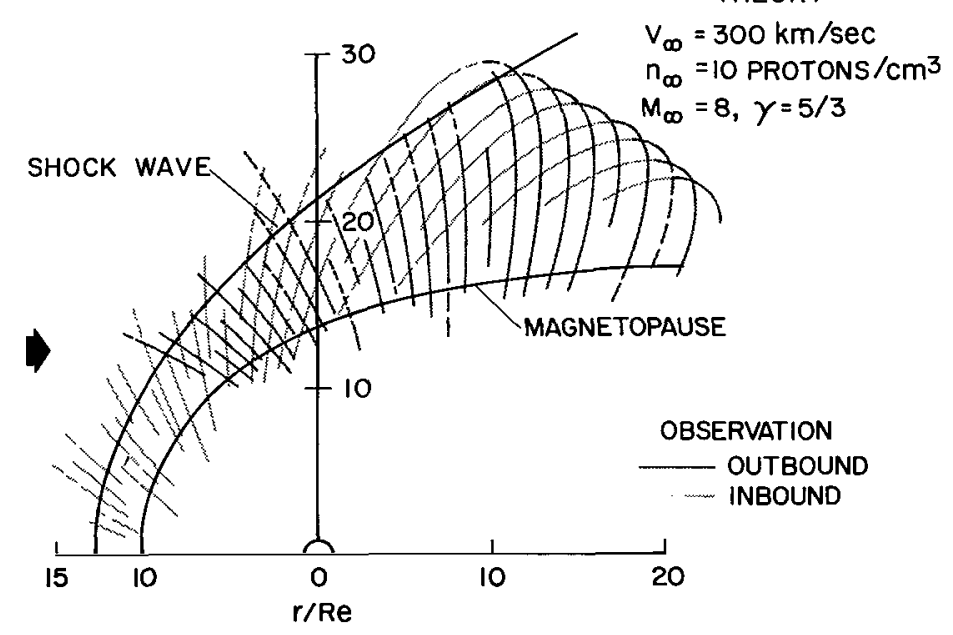

Figure 45. - IMP-1 plasma probe measurements of shock wave and magnetosphere boundary crossings.

in which are drawn the portions of the first 29 orbits in which Wolfe, Silva, and Myers (1966) interpret the data from the plasma probe to indicate that the spacecraft is between the magnetosphere boundary and the bow wave. The dashed portions of some of the orbital segments represent possible positions of the boundaries in cases in which the termination of the transition region was uncertain. As discussed previously in the presentation of results from Explorer 12, such uncertainties could be due either to the gradual character of the change or the occurrence of multiple crossings resulting from the magnetosphere boundary or shock wave moving back and forth past the spacecraft. This plot was derived by locating the transition region in terms of geocentric 
distance and solar oriented ecliptic longitude. It therefore does not account for the latitude of the spacecraft during its traversal through this region.

Superposed on the data are the theoretical positions for the magnetosphere boundary and the bow wave calculated for flow with free-stream Mach number $M_{\infty}=8$ and ratio of specified heats $\gamma=5 / 3$. The bulk velocity $v_{\infty}$ and density $n_{\infty}$ of the solar wind are indicated to be $300 \mathrm{~km} / \mathrm{sec}$ and 10 protons $/ \mathrm{cm}^{3}$, although any other combination of values having the same product $\mathrm{n}_{\infty} \mathrm{v}_{\infty}{ }^{2}$ would lead to identical curves for the magnetosphere boundary and the bow wave. Although the wide gaps between the energy channels and the limited directional information obtained by the plasma probe on IMP-1 prevent deduction of precise values for the density and bulk velocity of the solar wind, and hence the determination of detailed comparisons with theoretical results, the overall agreement of the gross features of the correspondence between the theoretical and observational results are clearly discernible in the results shown in figure 45. Although the criterion used to distinguish the transition region from the magnetosphere or the undisturbed solar wind are, of course, different, the same conclusion has been reached through examination of very similar appearing plots based on the magnetometer data from IMP-1 (see, for example, Ness, Scearce, and Seek (1964) or Ness, Scearce, Seek, and Wilcox (1966) for extensive accounts).

Although perhaps not so central to the present discussion of the external aerodynamics of the magnetosphere, mention should be made of a number of related features of the solar wind and the magnetosphere revealed by the data from IMP-1. One of the most striking of these is the existence of a long magnetic tail of the Earth and an enclosed magnetically neutral sheet extending far downstream from the Earth in the antisolar direction. Ness (1965) has reported that the diameter of the tail is approximately 40 Earth radii, and that magnetic field intensities of approximately 10 to 30 gammas are observed out to the satellite apogee of 31.7 Earth radii. The former is in deceptively good accordance with the theoretical results based on the Chapman-Ferraro theory, but the intensity and orientation of the magnetic field within the tail are very different from the current-free configuration visualized throughout the magnetosphere in that theory. The discovery of the neutral sheet and the attendant possibility of plasma instabilities have resulted in a vigorous development of theories to explain an extensive array of phenomena such as the aurora, gegenschein, day-night asymmetries of various sorts, and the formation of the Van Allen radiation belts. Accounts of many of these may be found in several other articles in this book.

Since the magnetosphere tail displayed no appreciable attenuation out to apogee, data from IMP-1 could provide no insight into its ultimate length. More recently, however, Ness, Behannon, Cantarano, and Scearce (1967) have reported that both the tail and a relatively thin magnetic neutral sheet have been observed in the data from Explorer 33 satellite at geocentric distances as great as about 80 Earth radii. Behannon (1968) has also reported that data from the same satellite showed the Earth's bow wave to be still detectable at a geocentric distance of 75.7 Earth radii. The probable, although less certain, detection of the geomagnetic tail at even greater distances ranging between 900 to 1050 Earth radii has been indicated by the plasma probe (Wolfe, Silva, McKibbin, and Mason, 1967) and magnetometer (Ness, Scearce, and 
Cantarano, 1967) data from Pioneer 7. A coherent, we11-ordered tail with embedded neutral sheet does not appear to have been observed, however, and it may be that the tail should be considered to be more like an irregular wake at this distance. Such an interpretation is consistent with the fact that measurements made by Mariner 4 spacecraft on a trajectory to Mars did not reveal any recognizable trace of the magnetosphere tail or wake as it passed through the anticipated region at a geocentric distance of about 3300 Earth radii (Van Allen, 1965).

Data from IMP-1 satellite were also valuable in confirming over an extending interval of time the general Archimedean spiral structure of the average interplanetary magnetic field as indicated previously by solar wind theory and by more limited measurements made in space, particularly by Mariner 2 (Smith, 1964). Particularly significant is the recognition by Wilcox and Ness (1965) of a long-lived magnetic sector structure corotating with the Sun in the interplanetary medium. The boundaries of the sectors were frequently sharply defined in the magnetic records, but less so in the data from the plasma probes and other instruments. In general, enhanced values for the intensity of the interplanetary magnetic field, the solar-wind velocity, and geomagnetic activity tend to be associated with the leading portion of each sector, and small values with the trailing portion. On the other hand, the density of the solar wind tends to be large in the leading and trailing portions of each sector, and small in the middle. The importance of these findings to the understanding of the 1ong-recognized 27-day recurrence pattern of geomagnetic storms is immediately evident.

More detailed information on the physical changes in the solar wind plasma as it crosses the Earth's bow shock wave has been presented recently by Argo, Asbridge, Bame, Hundhausen, and Strong (1967) using data from plasma probes on two Vela 3 satellites obtained on 13 shock crossings during July and August 1965. These satellites, which are in nearly circular orbits tilted at $60^{\circ}$ to the ecliptic plane, usually crossed the bow wave during this period at about $60^{\circ}$ and $300^{\circ}$ solar ecliptic longitude, and between $\pm 56^{\circ}$ ecliptic latitude, and at geocentric distances of approximately 18.5 Earth radii. In addition to presenting the results for each crossing, the data were summarized by giving average values for the ratios of the velocities, temperatures, and densities on the two sides of the shock. They are $v / v_{\infty}=0.70, T / T_{\infty}=24$, $\rho / \rho_{\infty}=3.4$, in which the subscript $\infty$ refers to conditions in the incident solar wind and the symbol without subscript refers to conditions behind the bow wave. The corresponding theoretical values of Spreiter, Summers, and Alksne (1966) at the positions of the Vela shock crossings are quoted as $\mathrm{v} / \mathrm{v}_{\infty}=0.70, \mathrm{~T} / \mathrm{T}_{\infty}=12, \rho / \rho_{\infty}=4$, of which it is judged that only the temperature fails to agree closely. Inspection of the latter reference or figure 23 of this paper shows that this theoretical value for the temperature ratio is that associated with a free-stream Mach number $M_{m}=8$ and ratio of specific heats $Y=5 / 3$, the combination usually selected for illustrative purposes, and that a more accurate value for the density ratio for this case is about 3.8. The actual values for the speed and temperature of the incident solar wind listed for the 13 shock crossings correspond to substantially higher free-stream Mach number than 8 , however. As a rough measure, it may be noted that the average of the Mach numbers for the 13 crossings is 11.3 . If this 
value is used for the Mach number together with the value 0.70 for $v / v_{\infty}$, the expression for $\mathrm{T} / \mathrm{T}$ shown on figure 23 leads, for $\gamma=5 / 3$, to $\mathrm{T} / \mathrm{T}_{\infty}=22.7$. Alternatively, the individual observational values listed for the speed and temperature of the solar wind and the ratio $v / v_{\infty}$ across the shock may be used to calculate a theoretical value for $T / T_{\infty}$ for each crossing. Taking the average of these in the same way as the experimental values were averaged yields a temperature ratio of 23.8 instead of the originally quoted value of 12. The new value is, of course, in virtually perfect agreement with the experimental value of 24 , and tends to confirm the appropriateness of the value $5 / 3$ for the ratio of specific heats as well as the usefulness of the aerodynamic mode1 generally.

The successful launching of Pioneer VI spacecraft equipped with a plasma probe having higher resolution than related instruments on earlier spacecraft, as well as a magnetometer and other instruments, provided an opportunity to make more detailed comparisons between the theoretical and observed characteristics of the solar wind and its interaction with the geomagnetic field than previously possible. Nature cooperated by providing a period of exceptionally steady flow of the solar wind during the time the spacecraft was traversing the region between the magnetosphere and the bow wave. On Earth, the geomagnetic field was also very steady with the planetary index $A_{p}$ attaining a value of only 1 during the time of interest. The entire period surrounding the launch date was marked by low geomagnetic activity with the daily $\mathrm{K}_{\mathrm{p}}$ sums on December 15, 16, and 17 being 2-, 1+, and 3-, respectively.

Pioneer VI was launched on December 16, 1965, into an escape trajectory that remained very close to the plane of the ecliptic and appeared as illustrated in figure 46 when viewed looking southward from over the north pole. The data from both the magnetometer (Ness, Scearce, and Cantarano, 1966) and the plasma probe (Wolfe, Silva, and McKibbin, 1968) show that the spacecraft crossed the magnetosphere boundary at 1256 UT at a geocentric distance of about 12.8 Earth radii and a solar-ecliptic longitude of $76.3^{\circ}$. It crossed

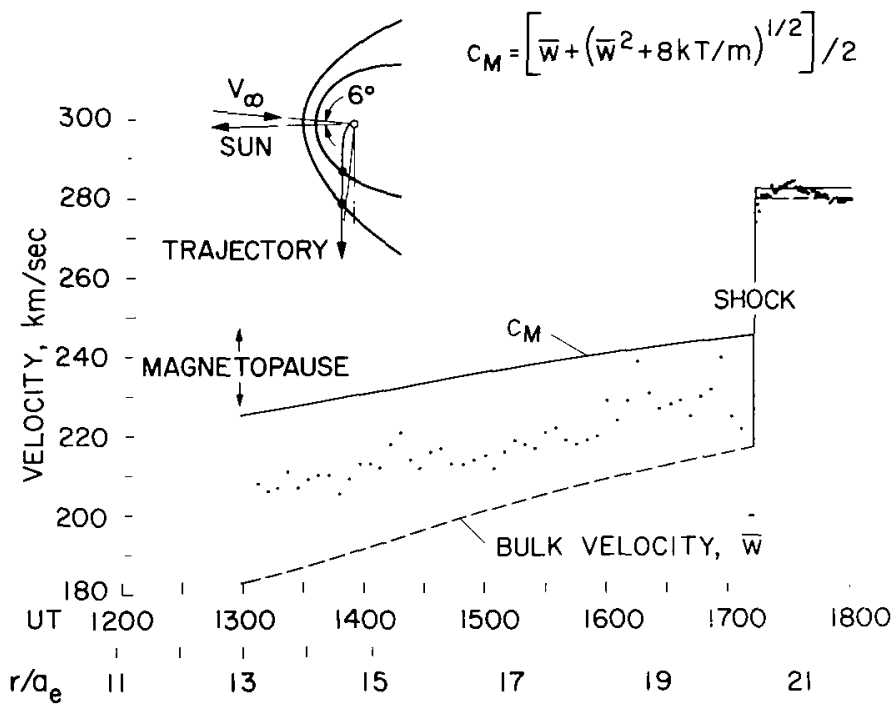

Figure 46.- Comparison of Pioneer VI peak flux ion velocity with calculated results for $M_{\infty}=8$ and $\gamma=5 / 3$. 
the bow wave somewhat more than four hours later at about 1712 UT when at a geocentric distance of approximately 20.5 Earth radii and solar-ecliptic longitude and latitude of $81.25^{\circ}$ and -4.40 , respectively.

Immediately after Pioneer VI passed the bow wave and emerged into the solar wind undisturbed by the presence of the Earth, the plasma probe detected a steady stream of particles coming from an apparent direction about $6^{\circ}$ west of the direction to the Sun with a speed of about $280 \mathrm{~km} / \mathrm{sec}$ and a number density of about 11 protons $/ \mathrm{cm}^{3}$ (and presumably an equal number of electrons). These values were used together with assumed values of 8 and $5 / 3$ for the freestream Mach number $M_{\infty}$ and the ratio of specific heats $\gamma$ to calculate the velocity and density of the flow and the intensity of the magnetic field all along the portion of the trajectory from inside the magnetosphere boundary to somewhat beyond the bow wave. The results are presented in figures 46,47 , and 48 together with those observed with Pioneer VI. The most apparent

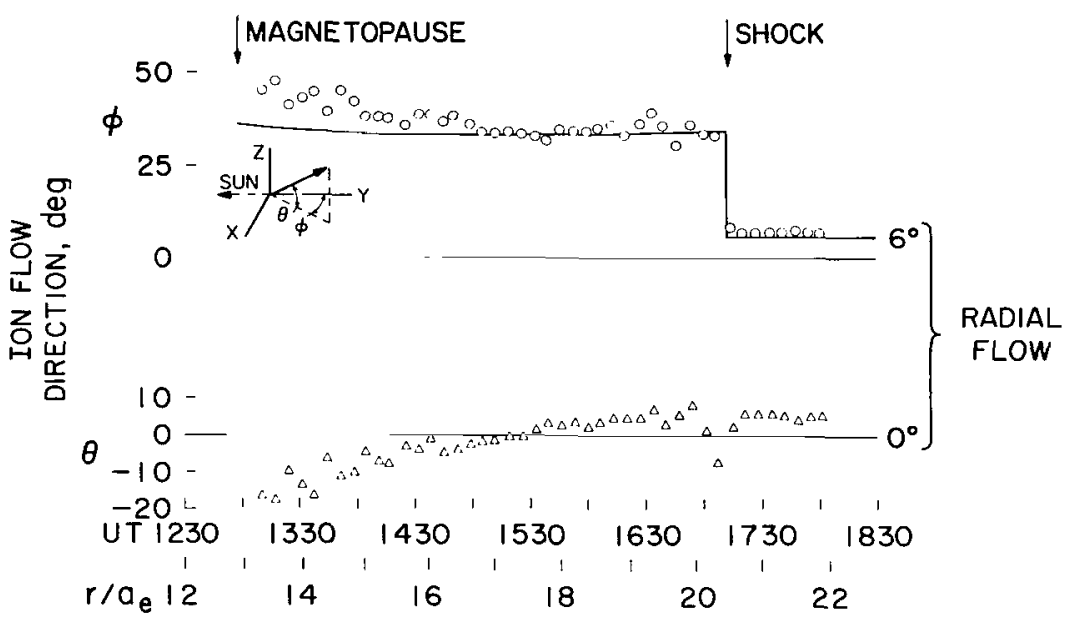

Figure 47.- Comparison of Pioneer VI flow direction with calculated results for $M_{\infty}=8, \gamma=5 / 3$.

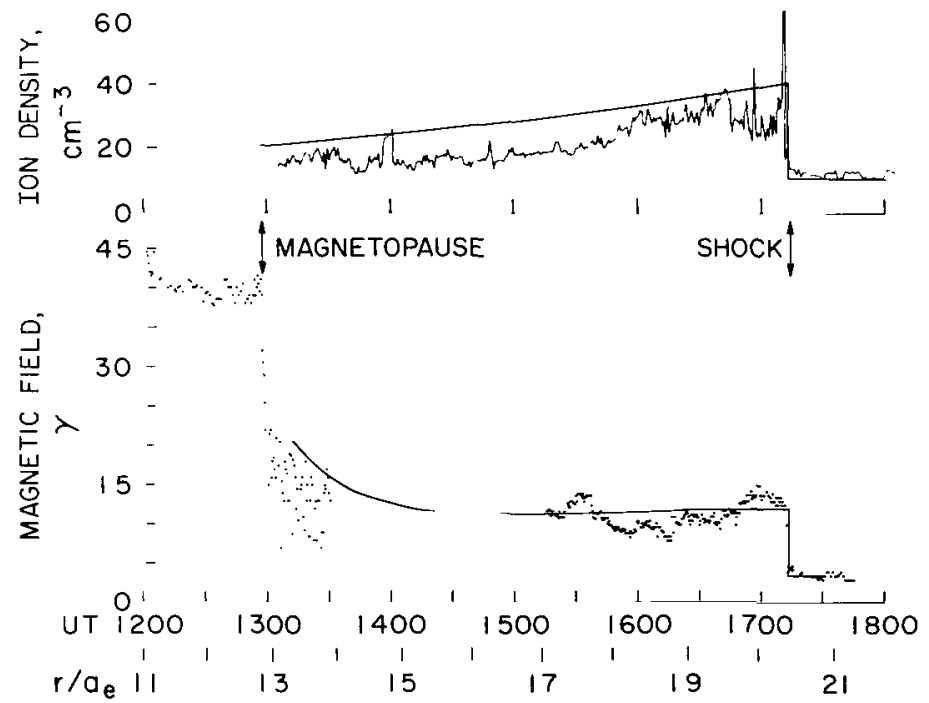

Figure 48.- Comparison of Pioneer VI ion density and magnetic field intensity with calculated results for $M_{\infty}=8, \gamma=5 / 3$. 
feature to be seen in the comparisons is the nearly perfect agreement between the calculated and measured locations of the magnetosphere boundary and the bow wave. It should be noted in this connection that a1though a particular value was selected for $M_{\infty}$ to make the calculations, the choice is not critical. As described previously, the locations of the magnetosphere boundary and the bow wave, and also the contours of constant $\rho / \rho_{\infty}, v / v_{\infty},|B| /\left|B_{\infty}\right|$, and $\left(T-T_{\infty}\right) / v_{\infty}{ }^{2}$, but not $T / T_{\infty}$, tend to be virtually independent of $M_{\infty}$ for free-stream Mach numbers greater than about 5 .

The observational data for the peak flux ion velocity during the time Pioneer VI traversed the transition region, and for about a half-hour thereafter, are presented in figure 46. In the incident solar wind where the random motions of the protons are small compared with their directed bulk velocity with respect to the Earth or the spacecraft, the peak flux ion velocity corresponds very nearly to the bulk velocity of the flow, as can be seen by comparing the values for $c_{M}$ and $\bar{w}=v$ for a somewhat different case in figure 33. Behind the bow wave, however, substantial differences emerge. In order to illustrate their significance more clearly, two sets of theoretical curves are included on figure 46 . The dashed line shows the bulk velocity calculated by direct application of the results for $v / v_{\infty}$ presented on figure 23 for a magnetosphere size determined in accordance with the results summarized in figure 15 for $v_{\infty}=280 \mathrm{~km} / \mathrm{sec}$ and $n_{\infty}=11$ protons $/ \mathrm{cm}^{3}$. The quantity represented by the data points is not the bulk velocity, however, but the velocity of the ions that produce the greatest current per unit energy increment in the plasma probe. Of the various quantities calculated theoretically, this peak flux ion velocity is most nearly comparable to the speed $c_{M}$ of the particles for which $\mathrm{dn} / \mathrm{n} \mathrm{dC} d \Omega$ is an absolute maximum. With the assumption that the solar wind is composed of fully ionized hydrogen, the relations given on figure 30 lead to the expression $c_{M}=\left[\bar{w}+\left(\bar{w}^{2}+8 k T / m\right)^{1 / 2}\right] / 2$ for $c_{M}$ in terms of the bulkvelocity $\bar{w}$ and the temperature $T$. The solid line on figure 46 indicates the values for the velocity $c_{M}$ computed in this way for the conditions stated above. In confirmation of the more qualitative statement above, it may be seen that the values for $\bar{w}$ and $c_{M}$ are nearly the same in the solar wind beyond the bow wave, and that substantial differences exist behind the shock wave where the temperatures are much higher and the bulk velocities somewhat lower than in the solar wind. In comparison, values for the peak flux ion velocity measured by Pioneer VI are slightly less than those calculated for $c_{M}$, although not so small as those calculated for the bulk velocity $\bar{w}$. With the estimated 10 -percent uncertainty in the determination of the experimental velocity distribution, however, the calculated results for both $c_{M}$ and $\bar{w}$ fall within the allowable range of values indicated by Pioneer VI. Aside from the matter of these modest differences in the absolute values, the general trend of slightly increasing velocity as the spacecraft moved along its trajectory from the magnetopause to the bow wave is represented very satisfactorily by the theoretical results.

Figure 47 shows the corresponding results for the calculated and observed direction of flow. The agreement is generally quite satisfactory, although some significant deviations between the calculated and measured results may be seen in the data obtained near the magnetopause. In these plots, the flow directions are given in terms of a coordinate system fixed relative to the 
Sun-Earth line as indicated in the small sketch. Because of the combined motion of the Earth and spacecraft, a flow that is radially outward from the Sun, as viewed in nonrotating heliocentric coordinates, would appear to approach the spacecraft with $\theta=0^{\circ}$ and $\varphi=6^{\circ}$ when $v_{\infty}=280 \mathrm{~km} / \mathrm{sec}$. It may be seen that the flow actually observed by Pioneer VI when beyond the bow wave is nearly in accordance with these values.

The corresponding comparison between the calculated and measured values for the density is shown in the top part of figure 48. Again the agreement is satisfactory. The plot in the lower part of the same figure shows the intensities of the magnetic field measured by Pioneer VI (Ness, Scearce, and Cantarano, 1966) and those calculated using the theoretical results of figure 24 together with the same values for $M_{\infty}, \gamma, v_{\infty}$, and $n_{\infty}$ as described above, and a value of 3.5 gamma for $B_{\infty}$ selected to fit the measurements in the solar wind. Considering the complexity of the calculation of the magnetic field as one of the last quantities to be evaluated in a long chain of calculations involving approximations at each step, the agreement is almost surprisingly good. It is, moreover, quite possible that some of the variations displayed by the observational data and not duplicated in the calculated results are the consequence of small changes in the intensity or direction of the interplanetary magnetic field, and could be satisfactorily reproduced by the theory if the interplanetary conditions were known.

One feature of the results for which the latter statement is probably not true, however, is the pronounced peak or spike in the run of values for the velocity, density, magnetic intensity, and $\theta$ component of the flow direction immediately downstream of the bow wave. The detection of such a localized irregularity is, moreover, not a unique property of the data from Pioneer VI, but a detail that has been repeatedly observed by other spacecraft in association both with the Earth's bow wave and with propagating interplanetary shock waves. Although continuum gasdynamic or magnetohydrodynamic calculations by themselves provide no indication of such behavior, it appears likely that these spikes can be understood in terms of the calculated properties of strong collisionless shock waves such as illustrated in figure 41 for Alfvén Mach numbers greater than 2 .

The preceding has not been intended to be an exhaustive or complete discussion of the contributions of the various satellites, but rather an account of some of the findings that bear on the assumptions and predictions of the theories of the solar wind and its interaction with the Earth. In particular, data from several Vela satellites discussed elsewhere in this book and a number of Soviet spacecraft have made important contributions, but space precludes further discussion here. In general, however, the results tend to be consistent with those described above, although certain features may be displayed with greater or lesser clarity depending upon the properties of the orbit, the type and resolution of the instruments, and conditions existing in space at the time of the flight. 


\section{SUMMARY OF STEADY ASPECTS OF THE INTERACTION OF THE SOLAR WIND AND THE GEOMAGNETIC FIELD}

Final1y, in broad outline, one may summarize many features of the steadystate hydromagnetic interaction of the solar wind and the magnetosphere in terms of the sketch shown in figure 49. The solar wind approaches the Earth with both the Mach number and the Alfvén Mach number much larger than unity,

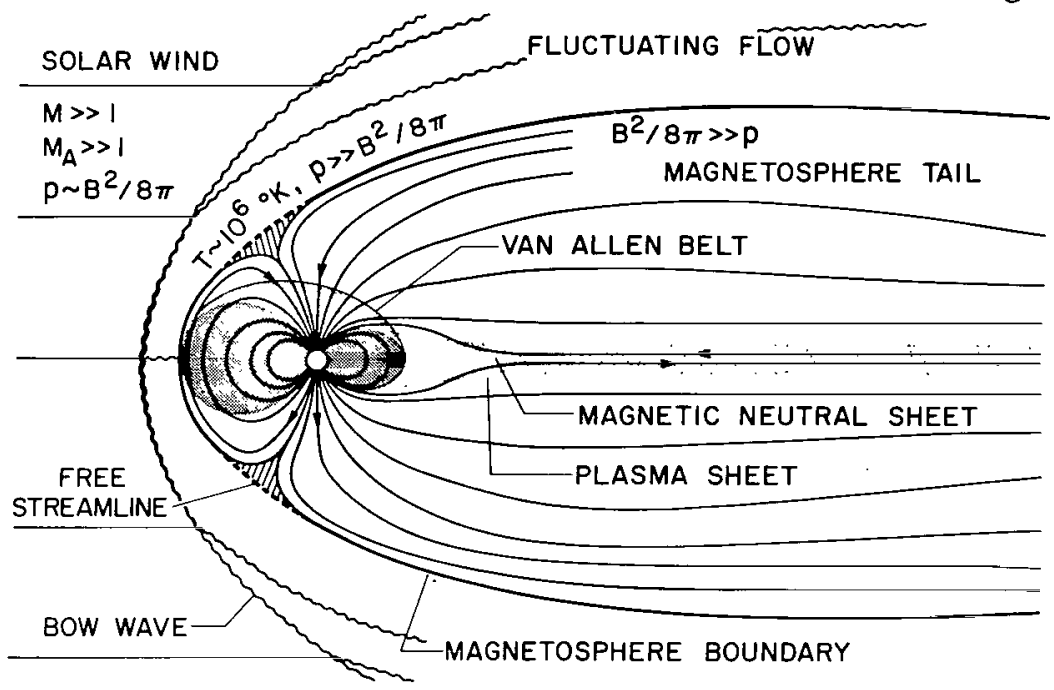

Figure 49.- The Earth as a magnetized obstacle in the solar wind.

and with the ratio of the gas pressure $p$ to the magnetic pressure $B^{2} / 8 \pi$ of the order unity. The high Alfvén Mach number assures that the equations of magnetohydrodynamics can be approximated with little error by the equations of gasdynamics for the flow and by the equations of frozen flux for the deformation of the interplanetary magnetic field. The high Mach number indicates that the flow is similar to a supersonic or hypersonic flow and that a detached bow wave would be expected upstream of an obstacle. Behind the bow wave, the temperature and pressure are greatly enhanced over their values in the solar wind, while the magnetic field tends to be increased only moderately, and is even diminished in some regions. As a result, the gas pressure tends to be substantially greater than the magnetic pressure in the flow around the forward part of the magnetosphere. The Alfven Mach number tends to remain well above unity, although not so great as in the undisturbed solar wind.

Inside the magnetosphere, the magnetic pressure is many orders of magnitude greater than the gas pressure. The magnetosphere is bounded by a current sheath that adjusts its shape like a free surface so that the sum of the gas and magnetic pressure is the same on both sides at all points. Essentially, this is a balance between the gas pressure of the exterior flow and the magnetic pressure of the confined and deformed geomagnetic field. With the introduction of the Newtonian approximation for the pressure of the flowing plasma, the determination of the shape of the magnetosphere boundary reduces to the classical Chapman-Ferraro problem based on the quite different concepts of particle, rather than fluid, motion. An incompatible condition is 
disclosed in the vicinity of the neutral points, however, in which an adequacy of the Newtonian approximation allows the appearance of a sharply reflexed curvature in the shape of the magnetosphere boundary. Normally, a shock wave would be expected to form in the supersonic flow along such a surface. It is not possible for such a shock wave to extend to the magnetosphere boundary, however, because the associated jump in pressure cannot be matched by a jump in magnetic pressure in the stationary curl-free magnetic field characteristic of the magnetosphere. It is proposed, in view of the known behavior of fluid flows generally, that the flow separates from the boundary of the geomagnetic field and subsequently reattaches farther downstream. In this way there is formed an embedded region of hot, essentially stationary, plasma bounded partially by the free surface just described and partially by the cusped geometry of the magnetic field lines. Previous studies of the properties of similar cusped-geometry configurations considered for plasma containment for production of thermonuclear energy lead to the conclusion that a substantial flux of particles from this region leak into the magnetosphere from the vicinity of the end of the cusp.

Although this paper is not concerned primarily with conditions in the interior of the magnetosphere, four prominent features of this region are included on this sketch for completeness. First of al1, there is, of course, the approximately dipole geomagnetic field essential to the formation of the cavity, or in effect the obstacle, around which the solar wind must flow. Extending out to the magnetosphere boundary in the subsolar region, and to a lesser distance elsewhere, are the trapped charged particles of the extended Van Allen belt. Trailing far downstream from the Earth is the magnetosphere tail of great, although still undetermined, extent. Within this region the magnetic field is essentially rectilinear in form with the field vectors pointing away from the Earth in the southern half of the tail and toward the Earth in the northern half. The field diminishes in intensity and reverses in direction in a relatively thin and flat surface, the magnetic neutral sheet, that extends approximately down the center of the tail. The cross section of the tail thus resembles, at least for the first several tens of Earth radii, the Greek letter $\theta$ with the magnetic field being approximately uniform in intensity throughout, but directed oppositely in the two halves. A somewhat greater than usual amount of plasma appears to be characteristic of the region near the magnetic neutral sheet, although the precise relation between the extensiveness of the plasma sheet and magnetic neutral sheet still remains to be determined.

\section{UNSTEADY ASPECTS}

Although all of the preceding discussion is concerned with steady-state phenomena, it has long been known that important transient effects exist. It was, in fact, observations of transient geomagnetic variations that led Chapman and others over 40 years ago into the pioneering studies that paved the way for the rapid understanding of many aspects of the data measured in recent years with spacecraft. Two distinctly different classes of major 
irregularities in the interplanetary plasma flow are sketched on figure 50 . One is a large plasma cloud of indefinite form and size expelled from the Sun by a flare or other explosive event. The existence of such a phenomena had

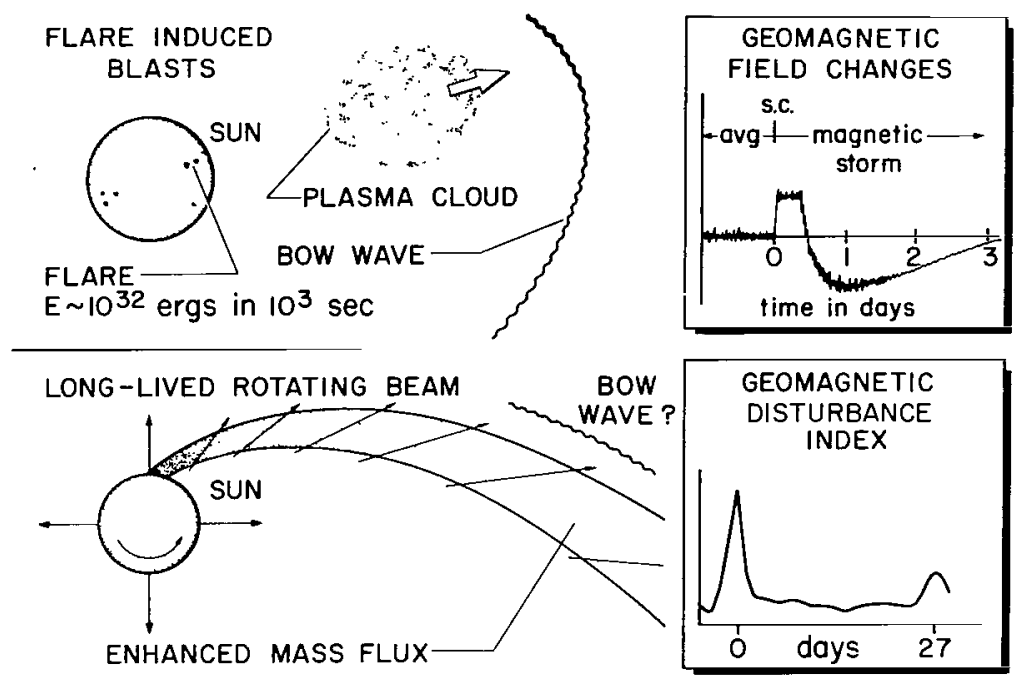

Figure 50.- Schematic drawings of two types of major irregularities in the solar wind and their geomagnetic consequences.

been suspected long before the launching of the first satellite because of the frequent observation of a flare on the surface of the Sun followed a few days later by a set of geophysical phenomena described collectively by the term "geomagnetic storm." One characteristic feature of such an event is that displayed in the upper right-hand part of figure 50 in which is depicted, in idealized form, the time variation of the horizontal component of the geomagnetic field observed in low and middle latitudes. The sudden commencement (s.c.) of many geomagnetic storms was originally thought to be the result of the impact of the plasma cloud on the geomagnetic field. More recently, however, the tendency has been to associate it more with the impact of a possible bow wave traveling through the solar wind in advance of the plasma cloud. The second class of major irregularity in the solar wind is a long-lived rotating beam, possibly preceded by a bow wave. This type of irregularity, called an M-region beam, had also long been suspected because of a pronounced tendency for geomagnetic storms to recur in approximately 27-day intervals with each complete rotation of the more active regions of the Sun. Earlier discussions of both the transient plasma clouds and the long-1ived rotating beams tended to regard them as intrusions into the otherwise effective vacuum of interplanetary space. Since the announcement of the theoretical likelihood and the verification of the actual existence of the solar wind at all times, both the plasma clouds and the beans must be regarded as moving through a general background of presumably less dense and slower moving flow of solar plasma.

Although spacecraft have now provided definitive evidence of the actual existence of such irregularities in the solar wind, their probable existence could have been inferred long ago from photographs of comets that occasionally display substantial changes in the direction of the tail in the course of a few hours. A famous pair of photographs illustrating such a change in the 
appearance of Comet Morehouse during a three-hour period on September 30, 1908, is shown in figure 51. Clearly visible is the substantial change in the direction of the tail much as though a front across which there is a sudden shift in the wind direction were moving along the length of the tail.
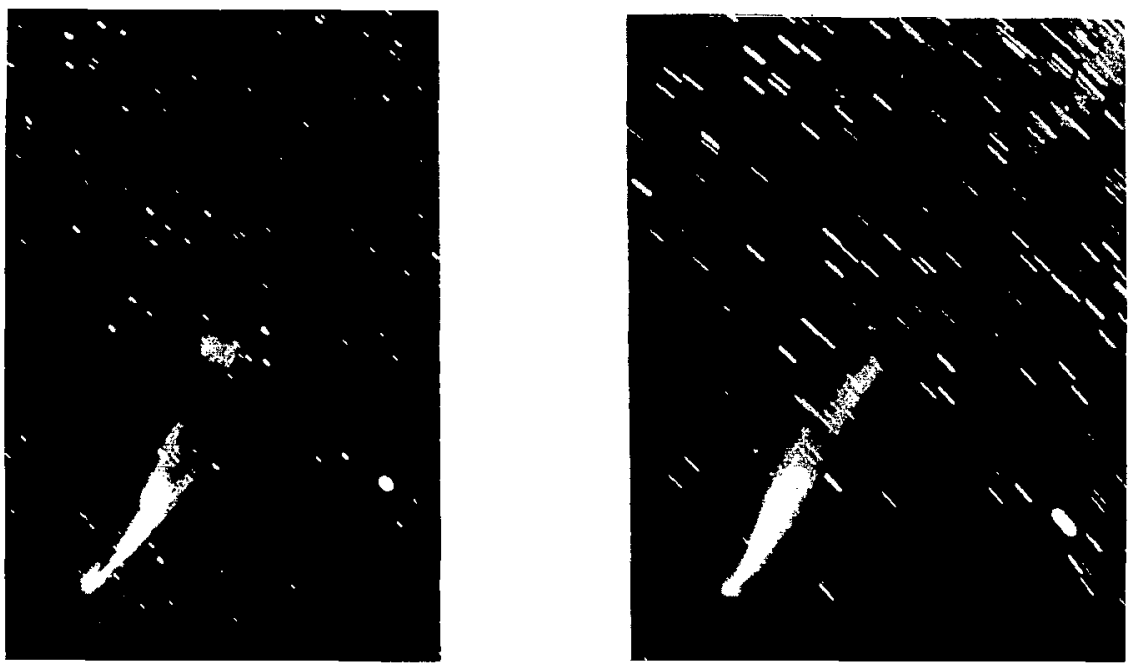

Figure 51.- Yerkes Observatory photographs of Comet 1908 III, Morehouse, showing change in 3 hours on September 30, 1908.

Impact of an irregularity of any sort in the solar wind upon the magnetosphere may be expected to initiate a variety of transient phenomena. Analysis of the steady-state theory indicates that a change in the momentum flux $\rho_{\infty} v_{\infty}{ }^{2}$ of the solar wind may be expected to result ultimately in a change in the size of the magnetosphere, and probably in the establishment of oscillations that would gradually damp with time. It is reasonably well ascertained observationally, although the precise details of the connecting mechanisms remain obscure, that such changes in the solar wind are also the cause, directly or indirectly, of several other important geophysical phenomena. Major fluctuations of the contents of the Van Allen belts may occur, accompanied by significant heating and enhanced ionization of the upper atmosphere sufficient, in more intense events, to impair radio communications, particularly in the polar regions. Auroral phenomena also appear to be affected significantly with increased activity and movement to lower latitudes being associated with certain phases of a geomagnetic storm.

In contrast to Chapman and Ferraro's concentration on transient phenomena, most of the more recent analyses of the interaction of solar plasma and the geomagnetic field, particularly those in which fluid models are invoked, are concerned with the steady-state problem. As a result, most of the quantitative theoretical analyses of large-scale transient features of the interaction are based on the older particle models that do not include the presence of a bow wave upstream of the magnetosphere. To the extent that disturbances do indeed propagate through the bow wave without excessive qualitative change, and the Newtonian formula, when expressed in a reference frame fixed with respect to an element of the magnetosphere boundary, continues to provide a reasonable approximation for the pressure, these results may still provide a guide to many features of the interaction. 
The rapidity with which the magnetosphere can respond to a major change in the solar wind is a matter of interest in many applications. Although the qualitative manner in which the geomagnetic field would carve a cavity in a finite cloud of solar plasma advancing through a vacuum toward the Earth was one of the first topics discussed by Chapman and Ferraro (1931), the actual quantitative solution of theix problem was not given until recently. In figures 52 and 53 are shown the results calculated numerically by Spreiter and Summers (1965) for two cases in which an extensive cloud of plasma having an initial density of 5 protons $/ \mathrm{cm}^{3}$ (and an equal number of electrons) and velocity of $500 \mathrm{~km} / \mathrm{sec}$ advances toward and engulfs the Earth. In both cases, the plasma cloud is assumed to have a flat face when at a geocentric distance of 20 Earth radii. In figure 52, this face is normal to the direction of motion; in figure 53, the plasma face is inclined $30^{\circ}$ thereto. The solid lines show the shape of the face of the plasma cloud in the equatorial plane at various times. The dashed lines show the final steady-state configuration of the boundary defined by the approximate solution described and illustrated in

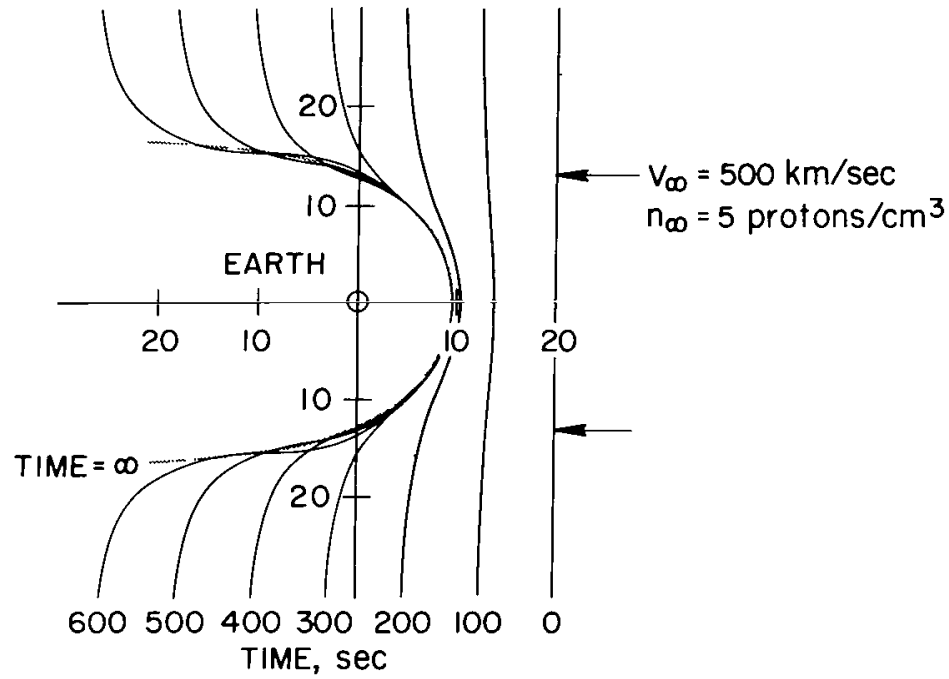

Figure 52.- Successive positions in equatorial plane of front of advancing plasma cloud.

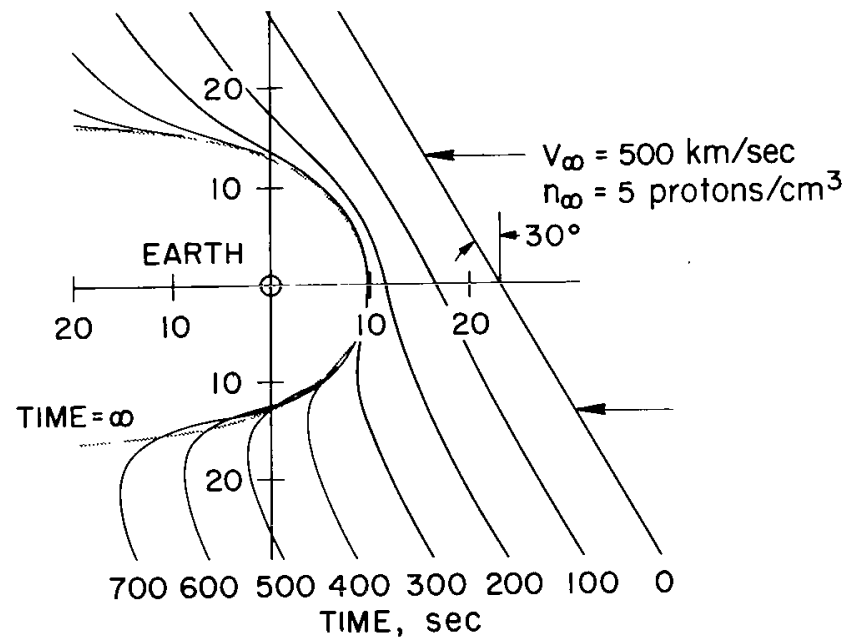

Figure 53. - Successive positions in equatorial plane of oblique front of advancing plasma cloud. figures 13 through 16 . The results are free of surprises and quite consistent with the early qualitative description of Chapman and Ferraro. Quantitative features of particular interest are the rapidity with which the boundary approaches its steadystate form as the plasma front sweeps by, the smallness of effects of obliquity of the plasma front, and the tendency to form waves that drift downstream along the boundary as it nears the steady-state configuration.

The results presented in dimensional form in figures 52 and 53 are convenient for providing a quick idea 
of the quantitative features of the interaction for a representative set of parameters, but lack generality as presented. The corresponding results for plasma clouds of speed and density different from those indicated may be determined quickly, however, by application of simple scaling laws inherent in the basic equations. They indicate that one must only alter the distance and time scales in accordance with the relations $r_{b} / r_{a}=\left(p_{s} t_{a} / p_{s} t_{b}\right)^{1 / 6}$ and $t_{b} / t_{a}=\left(p_{s} t_{a} / p_{s} t_{b}\right)^{1 / 6}\left(v_{\infty} / v_{\infty}\right)$ in which $p_{s t}=\rho_{\infty} v_{\infty}{ }^{2}$ is the stagnation pressure, subscript a refers to the values used to calculate figures 52 and 53 , and subscript $b$ refers to any other set of values.

The preceding problem is important because of its relative simplicity, conceptual value, and historical interest. The continual existence of the solar wind limits its actual usefulness, however. We consider next that a steady solar wind has existed for a sufficiently long time (of the order of 10 minutes or more on the basis of the preceding results) that the boundary separating the geomagnetic field and the solar plasma is well established in the steady-state configuration associated with a value $p_{s} t_{1}$ for the stagnation pressure. We consider further that a discontinuity in momentum flux advances through or with the solar wind plasma and that the discontinuity surface is planar over lateral distances larger than the magnetosphere before it begins to interact with the magnetic field of the Earth. Behind the discontinuity surface, conditions are considered to be steady and uniform, but are characterized by a larger stagnation pressure $p_{s} t_{2}$. Ultimately, therefore, the magnetosphere boundary will assume a configuration geometrically similar to that possessed originally, but smaller in the ratio $\left(\mathrm{p}_{\mathrm{st}} / \mathrm{p}_{\mathrm{st}}\right)^{1 / 6}$. The time history for the transition between the two steady-state configurations has been calculated by Spreiter and Summers (1965) and the results are shown in figure 54 for a case in which the initial steady state is the same as the final steady state arrived at in the case portrayed in figures 52 and 53 . The particular discontinuity considered is one that drifts along with the local velocity of the solar wind and across which there is a fourfold change in the density. The initial and final steady-state solutions are indicated by the

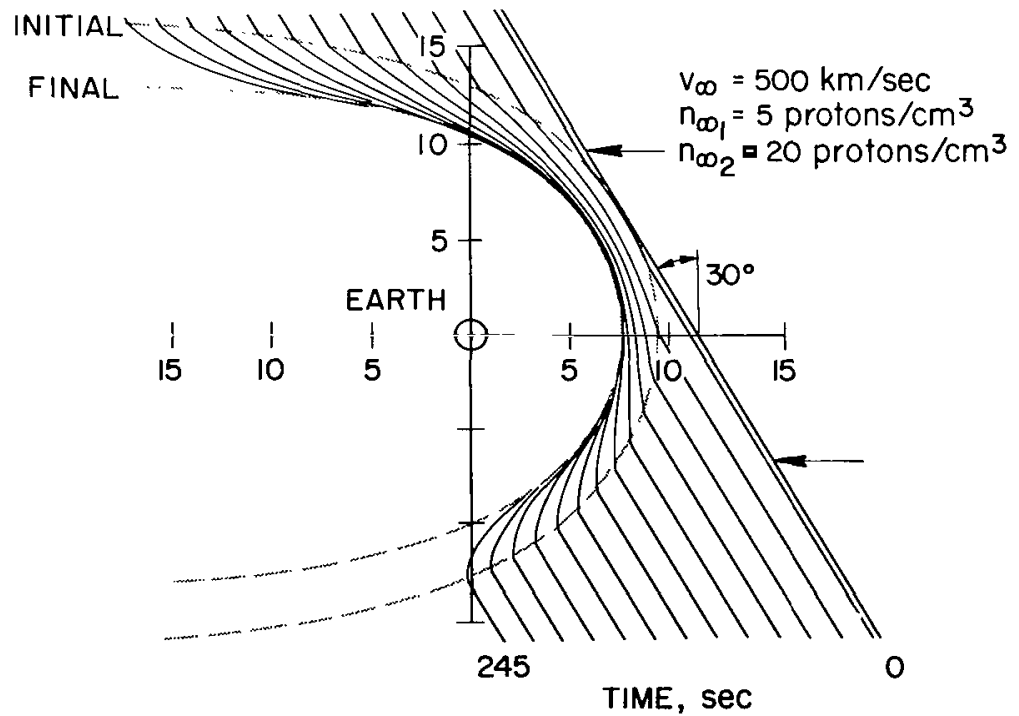

Figure 54.- Successive positions in equatorial plane of oblique density discontinuity in solar wind. 
outer and inner dashed lines. As in the previous examples, the solid lines represent the shape of the discontinuity surface at the stated times. The magnetosphere boundary at each of these times is given in part by the portion of the solid line that is interior to the outer dashed line. The remainder of the magnetosphere boundary is still stationary in this approximation in the initial position indicated by the outer dashed curve. As in the previous examples, the results show that the magnetosphere boundary collapses rapidly to its final configuration. Although several minutes are required for the discontinuity surface to sweep past the forward part of the magnetosphere, each element of the boundary essentially completes its movement from initial to final position in less than a minute.

It is not difficult to visualize the fluid counterparts of the problems described above, or to formulate their mathematical representation using the equations of magnetohydrodynamics. Because of the formidable difficulties encountered in the solution of these equations, however, quantitative discussion of such problems has hardly begun. Dryer, Merritt, and Aronson (1967) have described certain aspects of the dynamic interaction between the magnetosphere and bow wave system and a blast wave propagating through the solar wind with the aid of a laboratory experiment in which a blast wave produced by a shock tube is directed downstream through an operating supersonic wind tunnel containing a simplified model of the magnetosphere in the test section.

More localized aspects of the interaction such as the initial changes in a gasdynamic or magnetohydrodynamic shock wave or other discontinuity upon impact with the bow wave can be treated immediately by standard methods described in texts such as Courant and Friedrichs (1948), Landau and Lifshitz (1959), Oswatitsch (1956), and Jeffrey and Taniuti (1964). While methods, and even solutions in some cases, are immediately available from these and other sources, their application to the magnetosphere remains largely unexploited. As an example of the ease of such an application, we may consider the amplification of a plane sound wave upon head-on impact with a strong shock wave. Such an idealization might be considered sufficient to make the first estimates of what happens to a small disturbance in the solar wind upon encountering the forward part of the Earth's bow wave. For this problem, Landau and Lifshitz (1959) show that the shock wave is set into oscillation, and that both a sound wave and a contact surface wave are to be found in the region downstream from the shock wave. Also shown is that the ratio of the amplitude $\delta p_{2}^{\prime}$ of the pressure oscillations in the transmitted sound wave to the amplitude $\delta \mathrm{p}_{1}$ of the incident sound is proportional to the pressure ratio across the stationary shock wave. More precisely,

$$
\delta \mathrm{p}_{2}^{1} / \delta \mathrm{p}_{1}=\left\{\gamma+[2 \gamma(\gamma-1)]^{1 / 2}\right\}^{-1}\left(\mathrm{p}_{2} / \mathrm{p}_{1}\right)
$$

or approximately $0.317 \mathrm{p}_{2} / \mathrm{p}_{1}$ for $\gamma=5 / 3$. For a strong shock wave, $\mathrm{p}_{2} / \mathrm{p}_{1}=2 \gamma \mathrm{M}_{1}^{2} /(\gamma+1)$, or $5 \mathrm{M}_{1}^{2} / 4$ for $\gamma=5 / 3$, in which $\mathrm{M}_{1}$ should be equated to the incident Mach number based on the component of velocity normal to the shock wave. With $M_{1}=8$, as might occur at the nose of the Earth's bow wave, these expressions indicate that $\mathrm{p}_{2} / \mathrm{p}_{1}=80$ and $\delta \mathrm{p}_{2}^{\prime} / \delta \mathrm{p}_{1}=25.4$. This result illustrates the immense amplification that small disturbances may experience as they propagate across the Earth's bow wave. It also furnishes a possible alternative explanation to that of collisionless shock wave theory illustrated 
in the small inserts of figure 41 for the increased irregularities in the data from plasma probes and magnetometers behind the bow wave compared with corresponding data obtained in the incident solar wind. The different speeds of propagation of the transmitted sound wave and the contact surface wave produced by interaction of a sound wave and the bow wave offers the possibility of further richness of consequences because of interactions that may occur between the two transmitted wave systems. Burgers (1946) has shown, for example, that there will be a simple wave train of velocity and pressure disturbances propagating at the local speed of sound, and also a simple wave train of entropy disturbances carried along with the local velocity of the flow. The interaction of the density and temperature oscillations associated with both of these wave trains leads, in addition, to the development of oscillations with new frequencies by a process similar to that by which beats are produced. Details of the interaction of the bow wave and the wide variety of waves and other irregularities possible in the solar wind plasma remain largely undetermined, as do the characteristics of their propagation through the flow field exterior to the magnetosphere, and beyond to the interior of the magnetosphere and ultimately to the surface of the Earth.

The structure of the bow wave remains a topic surrounded by considerable uncertainties. Attention has been given in this text to the possibility that the structure may be understood in terms of the collisionless shock wave calculations of Auer, Hurwitz, and Kilb (1961, 1962) and Rossow $(1965,1967)$. Another possibility has been developed extensively recently by Tidman (1967a, b) in which a turbulent shock wave is considered to be formed through the action of ion waves produced as a result of two-stream instabilities of interpenetrating ion streams. In spite of these and several other proposals, it does not seem unfair to conclude that the precise determination of the detailed mechanism by which the bow wave is formed in the collisionless plasma of the solar wind remains largely unknown at the present time.

The stability of the magnetosphere boundary is another topic that has not been touched upon in the preceding discussion. It is apparent that the boundary must be stable in at least some gross sense, since otherwise the generally good agreement between observations and the steady-state theory could hardly be expected to occur. Numerous theoretical studies of the question of the stability of the magnetosphere boundary have been published over the last 10 years or more, but the results are often contradictory. Dungey $(1958,1963)$ considered that the flow of solar plasma along the magnetosphere boundary would generate surface waves in the same way that the wind generates waves on water through the action of a Kelvin-Helmholtz instability. He and Parker (1958) gave a theory of such waves, and concluded that the magnetosphere boundary is unstable. The applicability of the results to the magnetosphere is not assured immediately, however, because of the neglect of known features of the phenomena such as the compressibility of the plasma, the curvature of the boundary, and the effects of nonlinear terms in the governing equations. Spreiter and Summers (1965) proceeded differently by basing their analysis on the strict application of the Chapman-Ferraro theory. They found that, if the wave length and amplitude are sufficiently small that curvature and secondorder effects can be disregarded, all perturbations, except those having wave fronts alined with the direction of the local magnetic field within the magnetosphere, damp exponentially with time and drift along the magnetosphere 
boundary with the tangential component of the solar wind velocity. Alined waves, which neither damp nor amplify in this approximation, are examined further by inclusion of curvature and higher order effects. Analysis shows that curvature introduces a destabilizing effect in small regions of opposite to normal magnetosphere boundary curvature in the vicinity of the neutral points and a stabilizing effect elsewhere. With the elimination of the regions of reverse curvature near the neutral points, as described in connection with figure 39, even this exceptional region of instability disappears. Buffalano (1967) has considered this same model further, and shown that convection of a boundary perturbation along the magnetosphere boundary acts also to limit the growth of any instability. On the other hand, several others including Talwar (1964), Sen (1965), Lerche (1966) have developed analyses based on fluid concepts, and shown, for assumed conditions that may or may not adequately represent the actual conditions, that the magnetosphere boundary is unstable. More extensive accounts of this important and controversial subject have been given recently by Atkinson and Watanabe (1966) and Buffalano (1967).

\section{CONCLUDING REMARKS}

Three broad conclusions emerge from this review of the external aerodynamics of the magnetosphere. One is that substantial progress is being made in the development of methods for the calculation of the properties of both the solar wind and its interaction with the geomagnetic field. Another is that calculations based on fluid concepts of magnetohydrodynamics and gasdynamics are consistently of almost surprising accuracy considering both the questions surrounding the use of continuum methods and the introduction of simplifying assumptions necessary to achieve solutions at this time. Finally, one cannot avoid the conclusion that a considerable amount of further analysis is required to bring a corresponding clarification to many problems that still remain unsolved.

In the solar wind, the true nature of the heating mechanism in the corona that drives the solar plasma away from the Sun remains unknown in detail. A discussion of the manner by which the solar wind terminates at great distances from the Sun and merges with the interstellar medium, perhaps through the action of one or more terminal shock waves, has only begun. The ultimate consequences of such inquiry may be of considerable importance in understanding the interaction of stars and their gaseous environment in space. The quantitative analysis of the properties and propagation of transient effects is also of great interest and must be understood in much greater detail than at present for an adequate comprehension of a vast array of phenomena that come under the general heading of solar-terrestrial relations.

Any list of unsolved problems in the interaction of the solar wind and the geomagnetic field must include the quantitative description of the magnetosphere tail, and the development of a complete solution of the shape of the magnetosphere boundary and the properties of the surrounding flow in a completely self-consistent manner. It does not appear likely, however, that the solution of the latter very difficult problem would yield results substantially different from those that can now be calculated. An important problem 
in need of clarification is the question of the stability of the magnetosphere boundary. The structure of the bow wave and the mechanism by which it creates or amplifies irregularities in the flow is a topic of great intrinsic interest and important work needs to be done to provide even a rudimentary understanding. The properties and propagation of transient effects all the way from the solar wind, or even more ambitiously, from the surface of the Sun, to the Earth remains a subject of vital importance that is still relatively undeve1oped. Also of interest, although barely discussed as yet, is the extension and adaptation of the understanding achieved for the Earth to other objects in the solar system. This may possibly be straightforward in the case of a magnetized planet such as Jupiter, or very different in the case of essentially unmagnetized objects such as the Moon, Mars, and Venus.

Finally, perhaps the most intriguing question of all relates to the very success of the continuum models in dealing with the most rarefied gas presently accessible to direct measurement. It is "Why does collisionless, slightly magnetized plasma act so much like an ordinary collision-dominated gas?" It is not that one is entirely devoid of explanations, but those usually offered are somewhat unsatisfying and superficial involving little more than the simple replacement of the mean free path by some shorter interaction distance such as the gyro radius. The proper resolution of this question must certainly be ranked among the more important goals to be sought if the environment provided by interplanetary space is to live up to its frequently touted potential as a laboratory for the study of rarefied plasmas.

Ames Research Center

National Aeronautics and Space Administration

Moffett Field, Calif., 94035, October 27, 1967 $129-02-08-03-00-21$ 


\section{REFERENCES}

Alksne, A. Y. The steady-state magnetic field in the transition region between the magnetosphere and the bow shock. Planetary Space Sei. 15, $239-245,1967$.

Argo, H. V., Asbridge, J. R., Bame, S. J., Hundhausen, A. J., and Strong, I. B. Observations of solar wind plasma. Changes across the bow wave. J. Geophys. Res. 72, 1989-1994, 1967.

Atkinson, G., and Watanabe, T. Surface waves on the magnetospheric boundary as a possible origin of long period geomagnetic micropulsations. Earth Planetary Letters 1 , 89-91, 1966.

Auer, P. L., Hurwitz, H., Jr., and Kilb, R. W. Low Mach number magnetic compression waves in a collision-free plasma. Phys. Fluids 4, 1105-1121, 1961.

Auer, R. L., Hurwitz, H., Jr., and Kilb, R. W. Large-amplitude magnetic plasma. Phys. Fluids $5,298-316,1962$.

Beard, D. B. The interaction of the terrestrial magnetic field with the solar corpuscular radiation. J. Geophys. Res. 65, 3559-3568, 1960.

Behannon, K. W. Mapping of the earth's bow shock and magnetic tail by Explorer 33. J. Geophys. Res. 73, 907-930, 1968.

Belton, M. J. S., Brandt, J. C., and Hodge, P. W. On the characteristics of distant comets. Ann. Astrophys. 26, 250-252, 1963.

Biermann, L. Kometenschweife und solare korpuskular Strahlung. $Z$. Astrophys. 29, 274-286, 1951.

Biermann, L. Solar corpuscular radiation and the interplanetary gas. Observatory 77, 109-110, 1957.

Bondi, H. On spherically symmetrical accretion. Monthly Notices Roy. Astron. Soc. 112, 195-204, 1952 .

Brandt, J. C. A model of the interplanetary medium. Icams 1, 1-6, 1962 .

Brandt, J. C. Interplanetary gas XIII. Gross plasma velocities from the orientations of ionic comet tails. Astrophys. J. 147, 201-219, 1967.

Briggs, B. R., and Spreiter, J. R. Theoretical determination of the boundary and distortion of the geomagnetic field in a steady solar wind. NASA TR R-178, 1963.

Buffalano, C. A. Some problems pertaining to the macroscopic stability of the surface of the magnetosphere. Ph.D. Thesis, Yale University, Dept. Engineering and Applied Science, 1967. 
Burgers, J. M. Aerodynamics - On the transmission of sound waves through a shock wave. Koninklijke Adademie van Wetenschappen, Amsterdam, Proceedings, 44, 273-281, 1946.

Cahil1, L. J., and Amazeen, P. G. The boundary of the geomagnetic field. $J$. Geophys. Res. 68, 1835-1843, 1963.

Cahil1, L. J., Jr., and Pate1, V. L. The boundary of the geomagnetic field, August to November, 1961. Planetary Space Sci. 15, 997-1033, 1967.

Chamberlain, J. W. Interplanetary gas. III. A hydrodynamic model of the corona. Astrophys. J. 133, 675-687, 1961 .

Chapman, S. Solar plasma, geomagnetism and aurora. Geophysics, The Earth's Environment, edited by C. DeWitt, J. Hieblot, and A. Lebeau. Gordon and Breach, New York, pp. 371-502, 1963.

Chapman, S., and Ferraro, V. C. A. A new theory of magnetic storms. Terr. Magnetism Atmos. Elec. 36, 77-97, 171-186, 1931.

Cladis, J. B., Miller, T. D., and Baskett, J. R. Interaction of a supersonic plasma stream with a dipole magnetic field. J. Geophys. Res. 69, 2257-2272, 1964.

Courant, R., and Friedrichs, K. O. Supersonic Elow and Shock Waves. Interscience Pub., Inc., New York, 1948.

Davis, L., Jr., and Beard, D. B. A correction to the approximate condition for locating the boundary between a magnetic field and a plasma. $J$. Geophys. Res. 67, 4505-4507, 1962.

Dessler, A. J. Solar wind and interplanetary magnetic field. Rev. Geophys. 5, 1-41, 1967.

Dryer, M., and Heckman, G. R. On the hypersonic analogue as applied to planetary interaction with the solar plasma. Planetary Space Sci. 15 , $515-546,1967$.

Dryer, M., Merritt, D. L., and Aronson, P. M. Interaction of a plasma cloud with the earth's magnetosphere. J. Geophys. Res. $\underline{72}, 2955-2962,1967$.

Dungey, J. W. Cosmic EZectrodynomics. Cambridge University Press, 1958.

Dungey, J.W. The steady state of the Chapman-Ferraro problem in two dimensions. J. Geophys. Res. 66, 1043-1047, 1961.

Dungey, J.W. Structure of the exosphere or adventures in velocity space. Geophysics, The Earth's Environment, edited by C. DeWitt, J. Hieblot, and A. Lebeau. Gordon and Breach, New York, pp. 503-550, 1963.

Faus, A. A. Solar wind beyond the earth's orbit. The transition zone. Planetary Space Sci. 14, 143-154, 1966. 
Ferraro, V. C. A. On the theory of the first phase of a geomagnetic storm: A new illustrative calculation based on an idealized (plane not cylindrical) model field distribution. J. Geophys. Res. 57, 15-49, 1952.

Finch, H. F., and Leaton, B. R. The earth's main magnetic field - epoch 1955.0. Monthly Notices Roy. Astron. Soc., Geophys. Supp 2. 7, 314-317, 1957.

Freeman, J. W., Jr., Kavanagh, L. D., Jr., and Cahi11, L. J., Jr. An observation of transient variations in the magnetosphere boundary position. J. Geophys. Res. 72, 2040-2044, 1967.

Fuller, F. B. Numerical solutions for supersonic flow of an ideal gas around blunt two-dimensional bodies. NASA TN D-791, 1961.

Fukushima, N. Gross character of geomagnetic disturbance during the international geophysical year and the second polar year. Report of Ionosphere and Space Research in Japan XVI, pt. 1, 37-56, 1962.

Grad, H. Containment in cusped plasma systems. Plasma Physics and Thermonuclear Research, edited by C. L. Longmire, J. L. Tuck, and W. B.

Thompson. Pergamon Press, New York, pp. 189-200, 1963.

Grad, H., and Hu, P. N. Neutral point in the geomagnetic field. Phys. Fluids 9, 1610-1611, 1966.

Grossmann, W., Jr. Particle loss in a three-dimensional cusp. Phys. Fluids 9 , $2478-2485,1966$.

Hurley, J. Interaction of a streaming plasma with the magnetic field of a two-dimensional dipole. Phys. Fluids 4, 854-859, 1961.

Inouye, M., and Lomax, H. Comparison of experimental and numerical results for the flow of a perfect gas about blunt-nosed bodies. NASA TN D-1426, 1962 .

Jeffrey, A., and Taniuti, T. Non-Zinear Wave Propagation. Academic Press, New York; London, 1964.

Jensen, D. C., and Cain, J. C. An interim geomagnetic field (abstract) . J. Geophys. Res. 67, 3568-3569, 1962.

Jones, W. P., and Rossow, V. J. Graphical results for large-amplitude unsteady one-dimensional waves in magnetized collision-free plasmas with discrete structure. NASA TN D-2536, 1965.

Kaplan, S. A. InterstezZar Gasdynamics. Pergamon Press, Oxford, 1966.

Kaufmann, R. L. Shock observations with the Explorer 12 magnetometer. J. Geophys. Res. 72, 2323-2342, 1967. 
Kawashima, N., and Fukushima, N. Mode1 experiment for the interaction of solar plasma stream and geomagnetic field. Planetary Space Sci. 12, 1187-1201, 1964.

Kendal1, P. C. On the detailed shape of the Chapman-Ferraro surface. $J$. Atmospheric Terrest. Phys. 24, 860-863, 1962.

Landau, L. D., and Lifshitz, E. M. FZuid Mechanics. Addison-Wesley Pub. Co., Reading, Mass., 1959.

Lebeau, A. Sur l'activité magnétique diurne dans les calottes polaires. Ann. Geophys. 21, 167-218, 1965 .

Lerche, I. Validity of the hydromagnetic approach in discussing instability of the magnetosphere boundary. J. Geophys. Res. 71, 2365-2371, 1966 .

Levy, R. H., Petschek, H. E., and Siscoe, G. L. Aerodynamic aspects of the magnetospheric flow. AIAA J. 2, 2065-2076, 1964.

Lincoln, J. V. Geomagnetic and solar data. J. Geophys. Res. 67, 381-387, 1962.

Lomax, H., and Inouye, M. Numerical analysis of flow properties about blunt bodies moving at supersonic speeds in an equilibrium gas. NASA TR R-204, 1964 .

Lüst, R. Interplanetary plasma. Space Sci. Rev. ㅅ, 522-552, 1963.

Lüst, R. Interplanetary plasma. Introduction to Solar Terrestrial Relations, edited by J. Ortner and H. Maseland. Gordon and Breach, New York, pp. 181-209, 1965 .

McCrea, $W$. H. Shock waves in steady radial motion under gravity. Astrophys. J. 124, 461-468, 1956 .

Mead, G. D. Deformation of the geomagnetioc field by the solar wind. J. Geophys. Res. 69, 1181-1195, 1964.

Mead, G. D., and Beard, D. B. Shape of the geomagnetic field solar wind boundary. J. Geophys. Res. 69, 1169-1179, 1964.

Mead, G. D., and Cahill, L. J., Jr. Explorer 12 measurements of the distortion of the geomagnetic field by the solar wind. J. Geophys. Res. 72, $2737-2748,1967$.

Midgley, J. E., and Davis, L., Jr. Calculation by a moment technique of the perturbation of the geomagnetic field by the solar wind. $J$. Geophys. Res. 68, 5111-5123, 1963.

Ness, N. F. The earth's magnetic tail. J. Geophys. Res. 70, 2989-3005, 1965. 
Ness, N. F., Behannon, K. W., Cantarano, S. C., and Scearce, C. S. Observations of the earth's magnetic tail and neutral sheet at 510,000 kilometers by Explorer 33. J. Geophys. Res. 72, 927-933, 1967.

Ness, N. F., Scearce, C. S., and Cantarano, S. C. Preliminary results from Pioneer 6 magnetic field experiment. J. Geophys. Res. 71, 3305-3313, 1966.

Ness, N. F., Scearce, C. S., and Cantarano, S. C. Probable observations of the geomagnetic tail at $10^{3}$ earth radii by Pioneer 7 . J. Geophys. Res. 72, 3769-3776, 1967.

Ness, N. F., Scearce, C. S., and Seek, J. B. Initial results of the IMP-1 magnetic field experiment. J. Geophys. Res. 69, 3531-3569, 1964.

Ness, N. F., Scearce, C. S., Seek, J. B., and Wilcox, J. M. Summary of results from IMP-1 magnetic field experiment. Space Res. 6, 581-628, 1966.

Osborne, F. J. F., Bachynski, M. P., and Gore, J. V. Laboratory studies of the variation of the magnetosphere with solar wind properties. $J$. Geophys. Res. 69, 4441-4449, 1964.

Oswatitsch, K. Gasdynomics. Academic Press, New York, 1956.

Pai, G. L., and Sarabhai, V. A. Periodic fluctuations in the geomagnetic field during magnetic storms. PZanetary Space Sci. 12, 855-865, 1964.

Parker, E. N. Dynamics of the interplanetary gas and magnetic fields. Astrophys. J. 128, 664-676, 1958.

Parker, E. N. Interplanetary Dynamical Processes. Interscience Pub., New York, 1963.

Parker, E. N. Dynamical theory of the solar wind. Space Sci. Rev. 4 , $666-708,1965$.

Rossow, V. J. Magnetic compression of collision-free plasmas with charge separation. Phys. Fluids 8, 358-366, 1965.

Rossow, V. J. Magnetic-compression waves in collisionless plasma - oblique ambient magnetic field. Phys. Fluids 10, 1056-1062, 1967.

Scarf, F. L. The solar wind and its interaction with magnetic fields. Space Physics, edited by D. P. LeGalley and A. Rosen. John Wiley and Sons, Inc., pp. 437-473, 1964.

Sen, A. K. Stability of the magnetospheric boundary. PZanetary Space Sci. 13, $131-141,1965$.

Slutz, R. J., and Winkelman, J. R. Shape of the magnetospheric boundary under solar wind pressure. J. Geophys. Res. 69, 4933-4948, 1964. 
Smith, E. J. Interplanetary magnetic fields. Space Physics, edited by D. P. LeGalley and A. Rosen. John Wiley and Sons, Inc., 1964.

Smith, S. M., Henderson, M. E., and Torrey, R. A. Coronal photographs, isophotes, and a flash spectrum from the solar eclipse of May 30, 1965. NASA TN D-4012, 1967.

Sonnerup, B. U. Ö., and Cahil1, L. J., Jr. Magnetopause structure and attitude from Explorer 12 observations. J. Geophys. Res. 72, 171-183, 1967.

Spitzer, L., Jr. Physics of Fully Ionized Gases, Interscience Pub., Inc., New York, 1956; Second edition, 1962.

Spreiter, J. R., Alksne, A. Y., and Abraham-Shrauner, B. Theoretical proton velocity distributions in the flow around the magnetosphere. Planetary Space Sci. 14, 1207-1220, 1966.

Spreiter, J. R., and Briggs, B. R. Theoretical determination of the form of the hollow produced in the solar corpuscular stream by the interaction with the magnetic dipole field of the earth. NASA TR R-120, 1961.

Spreiter, J. R., and Briggs, B. R. Theoretical determination of the form of the boundary of the solar corpuscular stream produced by interaction with the magnetic dipole field of the earth. J. Geophys.Res. 67, 37-51, 1962 .

Spreiter, J. R., and Hyett, B. J. The effect of a uniform external pressure on the boundary of the geomagnetic field in a steady solar wind. $J$. Geophys. Res. 68, 1631-1642, 1963.

Spreiter, J. R., and Jones, W. P. On the effect of a weak interplanetary magnetic field on the interaction between the solar wind and the geomagnetic field. J. Geophys. Res. 68, 3555-3564, 1963.

Spreiter, J. R., and Summers, A. L. Effect of uniform external pressure and oblique incidence of the solar wind on the terminal shape of the geomagnetic field. NASA TR R-181, 1963.

Spreiter, J. R., and Summers, A. L. Dynamical behavior of magnetosphere boundary following impact by discontinuity in the solar wind. $J$. Atmospheric Terrest. Phys. 27, 357-365, 1965.

Spreiter, J. R., and Summers, A. L. On conditions near the neutral points on the magnetosphere boundary. Planetay Space Sci. 15, 787-798, 1967.

Spreiter, J. R., Summers, A. L., and Alksne, A. Y. Hydromagnetic flow around the magnetosphere. PZanetary Space Sci. 14, 223-253, 1966.

Strong, I. B., Asbridge, J. R., Bame, S. J., Heckman, H. H., and Hundhausen, A. J. Measurements of proton temperatures in the solar wind. Phys. Rev. Letters 16, 631-633, 1966 . 
Sturrock, P. A., and Hartle, R. E. Two-fluid model of the solar wind. Phys. Rev. Letters 16, 628-631, 1966.

Talwar, S. P. Hydromagnetic stability of the magnetospheric boundary. J. Geophys. Res. 69, 2707-2713, 1964.

Tidman, D. A. Turbulent shock waves in plasmas. Phys. Fluids 10, 547-564, $1967 \mathrm{a}$.

Tidman, D. A. The earth's bow shock wave. J. Geophys. Res. 72, 1799-1808, $1967 \mathrm{~b}$.

Van Allen, J.A. Absence of 40-KEV electrons in the earth's magnetospheric tail at 3300 earth radii. J. Geophys. Res. 70, 4731-4763, 1965.

Van Dyke, M. D. The supersonic blunt-body problem - review and extension. J. Aerospace Sci. $25,485-496,1958$.

Van Dyke, M. D., and Gordon, H. D. Supersonic flow past a family of blunt axisymmetric bodies. NASA TR R-1, 1959.

Walter, G. K. On the existence of a second standing shock wave attached to the magnetosphere. J. Geophys. Res. 71, 1341-1344, 1966.

Waniek, R. W., and Kasai, G. H. Interaction of a plasma flow with a threedimensional magnetic dipole. Proceedings of the 7th International Conference on Phenomena in Ionized Gases, Belgrad 2, 209-214, 1966.

Wilcox, J. M., and Ness, N. F. Quasi-stationary corotating structure in the interplanetary medium. J. Geophys. Res. 70, 5793-5805, 1965.

Wolfe, J. H., Silva, R. W., and McKibbin, D. D. Review of Ames Research Center plasma probe results from Pioneer 6 and Pioneer 7, 1968.

Presented at Summer Institute for Physics of the Magnetosphere, Boston College, Mass., June 19-28, 1967.

Wolfe, J.H., Silva, R. W., McKibbin, D. D., and Mason, R. H. Preliminary observation of a geomagnetospheric wake at 1000 earth radii. $J$. Geophys. Res. 72, 4577-4581, 1967.

Wolfe, J.H., Silva, R. W., and Myers, M. A. Observations of the solar wind during the flight of IMP-1. J. Geophys. Res. 71, 1319-1340, 1966.

Zhigulev, V. N., and Romishevskii, E. A. Concerning the interaction of currents flowing in a conducting medium with the earth's magnetic field. Soviet Phys. "DokZady" 4, 859-862, 1960. 


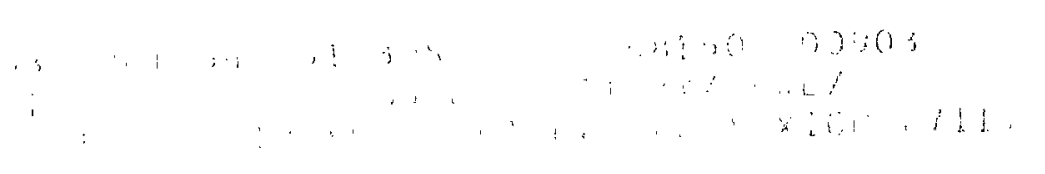

POSTMASTER: If Undeliverable (Section 158

"The aeronautical and space activities of the United States shall be conducted so as to contribute . . to the expansion of buman knowledge of phenomena in the atmosphere and space. The Administration shall provide for the widest practicable and appropriate dissemination of information concerning its activities and the results thereof."

\section{NASA SCIENTIFIC AND TECHNICAL PUBLICATIONS}

TECHNICAL REPORTS: Scientific and technical information considered important, complete, and a lasting contribution to existing knowledge.

TECHNICAL NOTES: Information less broad in scope but nevertheless of importance as a contribution to existing knowledge.

TECHNICAL MEMORANDUMS: Information receiving limited distribution because of preliminary data, security classification, or other reasons.

CONTRACTOR REPORTS: Scientific and technical information generated under a NASA contract or grant and considered an important contribution to existing knowledge.
TECHNICAL TRANSLATIONS: Information published in a foreign language considered to merit NASA distribution in English.

SPECIAL PUBLICATIONS: Information derived from or of value to NASA activities. Publications include conference proceedings, monographs, data compilations, handbooks, sourcebooks, and special bibliographies.

TECHNOLOGY UTILIZATION PUBLICATIONS: Information on technology used by NASA that may be of particular interest in commercial and other non-aerospace applications. Publications include Tech Briefs, Technology Utilization Reports and Notes, and Technology Surveys.

Details on the availability of these publications may be obtained from: 